\title{
Selective Acylation of Aryl- and Heteroarylmagnesium Reagents with Esters in Continuous Flow
}

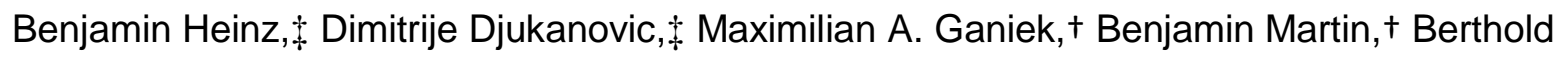 \\ Schenkel, + and Paul Knochel ${ }^{*}$
}

Department Chemie, Ludwig-Maximilians-Universität München,

Butenandtstr. 5-13, 81377 München, Germany

Paul.Knochel@cup.uni-muenchen.de

\section{Supporting Information}

\section{Table of Content}

General Information SI-2

Typical Procedures SI-4

Preparation of Products

Screening Results for the Reaction of $p$-Anisylmagnesium Bromide (2a) with Ethyl

Trifluoroacetate (7)

${ }^{1} \mathrm{H}-\mathrm{NMR},{ }^{19} \mathrm{~F}-\mathrm{NMR}$ and ${ }^{13} \mathrm{C}-\mathrm{NMR}$ Spectra of Products.

SI-31 


\section{General Information}

All reactions were carried out under argon or nitrogen atmosphere in glassware dried with a heat gun $\left(650^{\circ} \mathrm{C}\right)$ under high vacuum $(<1 \mathrm{mbar})$. Syringes which were used to transfer anhydrous solvents or reagents were purged thrice with argon or nitrogen prior to use. Indicated yields are isolated yields of compounds estimated to be $>95 \%$ pure as determined by ${ }^{1} \mathrm{H}-\mathrm{NMR}\left(25^{\circ} \mathrm{C}\right)$ and capillary GC. Unless otherwise indicated, all reagents were obtained from commercial sources.

\section{Solvents}

Solvents were dried according to standard procedures by distillation over drying agents and stored under argon.

THF was continuously refluxed and freshly distilled from sodium benzophenone ketyl under nitrogen.

Solvents for column chromatography were distilled on a rotary evaporator prior to use.

\section{Reagents}

IPrMgCl-LiCl: ${ }^{1}$ Magnesium turnings $(2.67 \mathrm{~g}, 110 \mathrm{mmol})$ and anhydrous $\mathrm{LiCl}$ (4.66 g, $100 \mathrm{mmol})$ were placed in an argon-flushed flask and THF $(50 \mathrm{~mL})$ was added. A solution of $\operatorname{PrCl}(9.13 \mathrm{~mL}, 100 \mathrm{mmol})$ in THF $(50 \mathrm{~mL})$ was slowly added at $25^{\circ} \mathrm{C}$. The reaction starts within a few minutes. After addition, the reaction mixture was stirred for $12 \mathrm{~h}$ at $25^{\circ} \mathrm{C}$.

The grey solution of $\mathrm{PrMgCl} \cdot \mathrm{LiCl}$ was cannulated to another flask under argon and removed in this way from excess of magnesium. A yield of ca. $95-98 \%$ of $\mathbb{P r M g C l} \cdot \mathrm{LiCl}$ is obtained. ${ }^{1}$

${ }^{1}$ A. Krasovskiy, P. Knochel, Angew. Chem. Int. Ed. 2004, 43, 3333-3336. 


\section{Content determination of organometallic reagents}

IPrMgCl. $\mathbf{L i C l}$ was titrated with $\mathrm{I}_{2}$ in $\mathrm{THF}^{2}$

Organomagnesium reagents were titrated with $\mathrm{I}_{2}$ in THF. ${ }^{2}$

\section{Flow reactions}

Flow reactions were carried out with solutions of the reactants in dry THF. Flame-dried glassware was used as reservoir for the reagent solutions and kept under an argon atmosphere during the reactions. Tetradecane was used as internal standard. For all flow reactions a Vapourtec E-series Integrated Flow Chemistry System with 3rd Pump Kit, Organometallic Kit, Collection Valve Kit and Cryogenic Reaction Kit was used. Reactions were performed in coiled tube reactors. Coiled reactors $(1.0,5.0,10.0$ or $20.0 \mathrm{~mL})$ were made from PFA or PTFE Teflon (i.d. $=0.8 \mathrm{~mm}, 0 . \mathrm{d}$. $=1.6 \mathrm{~mm}$ ). Prior to performing reactions, the system was dried by flushing it with dry THF (flow rate of all pumps: $1.00 \mathrm{~mL} \cdot \mathrm{min}^{-1}$; run-time: $30 \mathrm{~min}$ ).

\section{Chromatography}

Flash column chromatography was performed using silica gel $60(0.040-0.063 \mathrm{~mm})$ from MERCK.

Thin layer chromatography was performed using $\mathrm{SiO}_{2}$ pre-coated aluminum plates (Merck $60, \mathrm{~F}-254)$. The chromatograms were examined under $254 \mathrm{~nm}$ UV irradiation and/or by staining the TLC plate with a $\mathrm{KMnO}_{4}$ solution followed by heating with a heat gun.

HPLC was performed on an Agilent Technologies 1200 Series using a Chromolit® SemiPrep RP-18e 100-10 mm column. The HPLC was run with a gradient of acetonitrile/water.

\footnotetext{
${ }^{2}$ P. Knochel, A. Krasovskiy, Synthesis 2006, 2006, 890-891.
} 


\section{Analytical Data}

${ }^{1} \mathrm{H}-\mathrm{NMR}$ and ${ }^{13} \mathbf{C}$-NMR spectra were recorded on VARIAN Mercury 200, BRUKER ARX 300, VARIAN VXR $400 \mathrm{~S}$ and BRUKER AMX 600 instruments. Chemical shifts are reported as values in ppm relative to tetramethylsilane. $\mathrm{CDCl}_{3}$ peaks were set to $7.26 \mathrm{ppm}$ in ${ }^{1} \mathrm{H}$ NMR and $77.16 \mathrm{ppm}$ in ${ }^{13} \mathrm{C}$ NMR experiments. The following abbreviations were used to characterize signal multiplicities: s (singlet), d (doublet), dd (doublet of doublets), $t$ (triplet), q (quartet), hept (heptett) as well as $\mathrm{m}$ (multiplet).

Mass spectroscopy: High resolution (HRMS) and low resolution (MS) spectra were recorded on a FINNIGAN MAT 95Q instrument. Electron impact ionization (EI) was conducted with an ionization energy of $70 \mathrm{eV}$. For coupled gas chromatography/mass spectrometry, a HEWLETT-PACKARD HP 6890/MSD 5973 GC/MS system was used. Molecular fragments are reported starting at a relative intensity of $10-20 \%$.

Infrared spectra (IR) were recorded from $4500 \mathrm{~cm}^{-1}$ to $650 \mathrm{~cm}^{-1}$ on a PERKIN ELMER Spectrum BX-59343 instrument. For detection a SMITHS DETECTION DuraSamplIR II Diamond ATR sensor was used. The main absorption peaks are reported in $\mathrm{cm}^{-1}$.

Melting points (m.p.) were determined on a BÜCHI B-540 melting point apparatus and are uncorrected.

\section{Typical Procedures}

Typical Procedure 1: Preparation of organomagnesium reagents via Mg-insertion.

$\mathrm{LiCl}$ (509 mg, $12.0 \mathrm{mmol}, 1.2$ equiv) was flame dried and cooled to room temperature in vacuo. Then, magnesium turnings ( $288 \mathrm{mg}, 12.0 \mathrm{mmol}, 1.2$ equiv) and THF (10 mL) were added and the reaction mixture was cooled to $0^{\circ} \mathrm{C}$. The organic halide $(10.0 \mathrm{mmol}, 1.0$ equiv) was added dropwise and the reaction was stirred at $0^{\circ} \mathrm{C}$ for $1-3 \mathrm{~h}$. Upon completion of the insertion, the concentration was determined by titration against iodine in THF. 
Typical Procedure 2: Preparation of organomagnesium reagents via I-Mg exchange.

A dry and argon flushed Schlenk-flask equipped with a magnetic stirring bar and a septum was charged with the substituted aryl iodide $(10.0 \mathrm{mmol}, 1.0$ equiv) and dry THF (10 mL). The reaction mixture was cooled to the appropriate temperature before $i \mathrm{PrMgCl} \cdot \mathrm{LiCl}(11.0 \mathrm{mmol}$, 1.1 equiv.) was added dropwise. The progress of the iodine-magnesium exchange was monitored by GC-analysis of reaction aliquots quenched with aq. sat. $\mathrm{NH}_{4} \mathrm{Cl}$ solution. Upon completion of the exchange, concentration was determined by titration against iodine in THF.

Typical Procedure 3: Preparation of glycol esters of type 13 and subsequent formation to Mg-alkoxides of type 14.

Acyl chloride (25.0 mmol, 1.0 equiv) was added slowly to a stirring solution of ethylene glycol (4.20 mL, $75.0 \mathrm{mmol}, 3.0$ equiv) and pyridine (2.25 mL, $27.5 \mathrm{mmol}, 1.1$ equiv) in $25 \mathrm{~mL}$ of anhydrous dichloromethane at $0{ }^{\circ} \mathrm{C}$. After stirring for an additional $24 \mathrm{~h}$ at room temperature, the reaction was diluted with ethyl acetate $(200 \mathrm{~mL})$, washed with water $(3 \times 50 \mathrm{~mL})$ and brine $(50 \mathrm{~mL})$, dried over $\mathrm{Na}_{2} \mathrm{SO}_{4}$, filtered, and concentrated. Flash chromatographic purification over silica with the appropriate eluent afforded the monoacylated ethylene glycol derivatives of type 13.

A dry and argon flushed Schlenk-flask equipped with a magnetic stirring bar and a septum was charged with the monoacylated ethylene glycol (5.00 mmol, 1.0 equiv) and dry THF (16 $\mathrm{mL})$. The reaction mixture was cooled to $0{ }^{\circ} \mathrm{C}$, before $\mathrm{PrMgCl} \cdot \mathrm{LiCl}(5.00 \mathrm{mmol}, 1.0$ equiv.) was added dropwise. The reaction mixture was stirred for additional 5 min prior to use. 
Typical Procedure 4: Preparation of ketones starting from esters and organomagnesium reagents in flow.

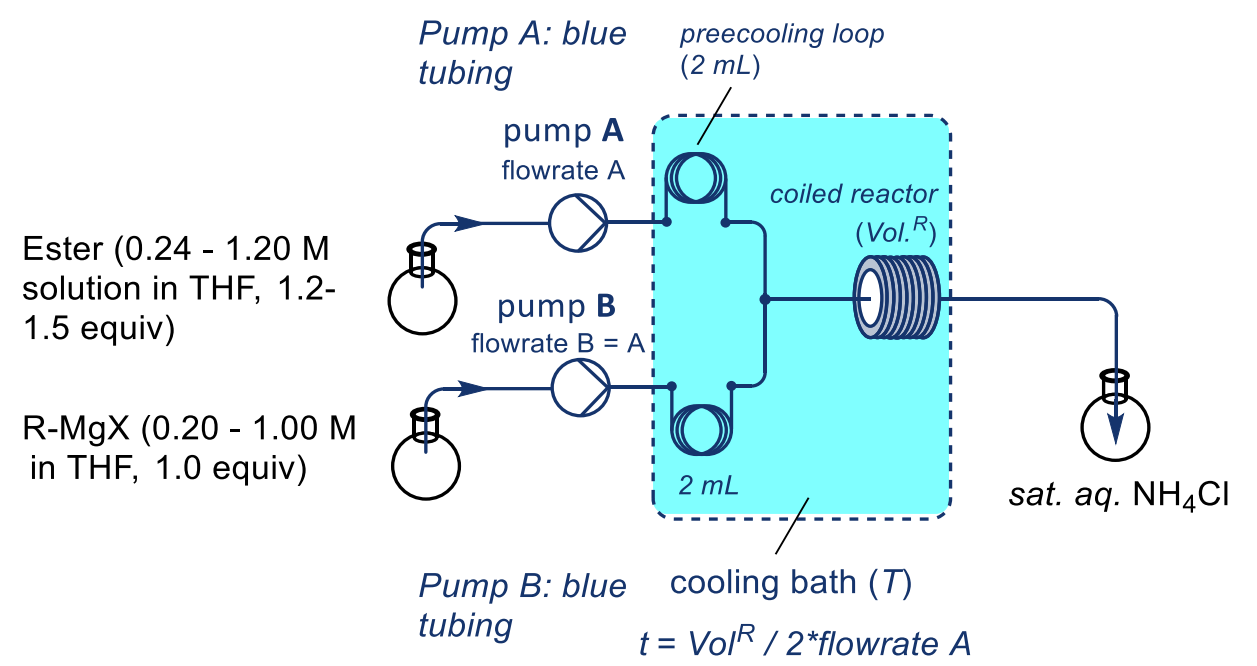

Figure SI1: Flow chemistry setup for preparation of ketones.

An organomagnesium reagent in $\operatorname{THF}(0.20-1.00 \mathrm{M}, 1.0$ equiv) and a solution of ester in THF (0.24 - 1.20 M, 1.2 equiv) were prepared. The solutions were pumped from their flasks through a suction needle at flowrate $A=1.0-5.0 \mathrm{~mL} \cdot \mathrm{min}^{-1}$ and flowrate $B=$ flowrate $A$. After passing a PTFE tubing (volpre $=2.0 \mathrm{~mL}, \mathrm{~T}=-5^{\circ} \mathrm{C}$ to $25^{\circ} \mathrm{C}$, residence time: $24-120 \mathrm{~s}$ ) for precooling, the solutions were mixed in a T-mixer ${ }^{3}$ (PFA or PTFE, I.D. $=0.5 \mathrm{~mm}$ ). The combined stream passed a PTFE reactor tube $\left(\mathrm{Vol}^{\mathrm{R}}=20 \mathrm{~mL}\right.$; residence time: $\mathrm{t}=2-10 \mathrm{~min}$, $\mathrm{T}=-5^{\circ} \mathrm{C}-25^{\circ} \mathrm{C}$ ) and was subsequently injected in a flask containing a stirred sat. aq. $\mathrm{NH}_{4} \mathrm{Cl}$ solution for quenching. The aqueous phase was extracted with EtOAc and the combined organic phases were dried over $\mathrm{Na}_{2} \mathrm{SO}_{4}$ and filtrated. After removal of the solvent in vacuo, flash column chromatographical purification with suited isohexane:EtOAc mixtures afforded the pure products.

\section{Batch Comparison Experiments}

An argon-flushed, flamedried flask was charged with ethyl trifluoroacetate (7) $(0.07 \mathrm{~mL}$, 1.2 equiv, $0.60 \mathrm{mmol})$ and THF $(1.2 \mathrm{~mL})$. After cooling the solutions to the desired temperature, (4-methoxyphenyl)magnesium bromide $(0,51 \mathrm{~mL}, 0.98 \mathrm{M}, 1.0$ equiv, $0.50 \mathrm{mmol})$ was added dropwise over $1 \mathrm{~min}$ to the stirred reaction mixture. The reaction was quenched after the appropriate time by adding sat. aq. $\mathrm{NH}_{4} \mathrm{Cl}$.

\footnotetext{
${ }^{3}$ The use of a Y-mixer (I.D. $=0.5 \mathrm{~mm}$ ) led to the same results.
} 


\section{Preparation of Products}

\section{2,2,2-Trifluoro-1-(4-methoxyphenyl)ethan-1-one (6a)}<smiles>COc1ccc(C(=O)C(F)(F)F)cc1</smiles>

Following TP4, precooled solutions of ethyl trifluoroacetate (7) (1.18 M, 1.2 equiv, $0.40 \mathrm{mmol})$ and (4-methoxyphenyl)magnesium bromide ( $0.98 \mathrm{M}, 1.0$ equiv, $0.33 \mathrm{mmol})$, prepared via TP1, were mixed in continuous flow (flowrate $A=5 \mathrm{~mL} \cdot \mathrm{min}^{-1}$, Vol ${ }^{R}=20 \mathrm{~mL}$, residence time: $t=2$ $\min , \mathrm{T}=-5^{\circ} \mathrm{C}$ ). Thereafter, the reaction mixture was quenched with $\mathrm{NH}_{4} \mathrm{Cl}$. After workup, the crude product was purified via column chromatography (isohexane:ethyl acetate $=9.5: 0.5$ ) to give $6 a(45.0 \mathrm{mg}, 0.22 \mathrm{mmol}, 67 \%)$ as a colorless solid.

${ }^{1} \mathrm{H}-\mathrm{NMR}\left(400 \mathrm{MHz}, \mathrm{CDCl}_{3}\right): \delta / \mathrm{ppm}=8.05(\mathrm{dq}, J=9.2,1.1 \mathrm{~Hz}, 2 \mathrm{H}), 7.00(\mathrm{~d}, J=9.1 \mathrm{~Hz}, 2 \mathrm{H})$, $3.91(\mathrm{~s}, 3 \mathrm{H})$.

${ }^{13} \mathrm{C}-N M R\left(101 \mathrm{MHz}, \mathrm{CDCl}_{3}\right): \delta / \mathrm{ppm}=178.9$ (q, $\left.J=34.5 \mathrm{~Hz}\right), 165.4,132.8,122.8,118.4$ (q, $J=291.5 \mathrm{~Hz}), 114.4,55.7$.

The spectra matched those of the literature. ${ }^{4}$

\section{Upscale Experiment for Compound 6a}

(4-Methoxyphenyl)magnesium bromide (0.50 M, 1.0 equiv, $2.00 \mathrm{mmol})$, prepared via TP1 and a solution of ethyl trifluoroacetate (7) in THF $(0.60 \mathrm{M}, 1.2$ equiv, $2.40 \mathrm{mmol})$ were prepared. The solutions were pumped from their flasks through a suction needle at flowrate $A=5.0 \mathrm{~mL} \cdot \mathrm{min}^{-1}$ and flowrate $B=$ flowrate $A$ (suction time $=48 \mathrm{sec}$ ). After passing a PTFE tubing (volpre $=2.0 \mathrm{~mL}, \mathrm{~T}=-5^{\circ} \mathrm{C}$, residence time: $24 \mathrm{~s}$ ) for precooling, the solutions were mixed in a T-mixer (PFA or PTFE, I.D. $=0.5 \mathrm{~mm}$ ). The combined stream passed a PTFE reactor tube $\left(\mathrm{Vol}{ }^{\mathrm{R}}=20 \mathrm{~mL}\right.$; residence time: $\left.\mathrm{t}=2 \mathrm{~min}, \mathrm{~T}=-5^{\circ} \mathrm{C}\right)$ and was subsequently injected in a flask containing a stirred sat. aq. $\mathrm{NH}_{4} \mathrm{Cl}$ solution for quenching at $25^{\circ} \mathrm{C}$. The aqueous phase was extracted with $\mathrm{Et}_{2} \mathrm{O}$ and the combined organic phases were dried over $\mathrm{Na}_{2} \mathrm{SO}_{4}$ and filtrated. After removal of the solvent in vacuo, flash column chromatographical purification

${ }^{4}$ T. Konno, T. Takehana, M. Mishima, T. Ishihara, J. Org. Chem. 2006, 71, 3545-3550. 
(isohexane:ethyl acetate $=9.5: 0.5)$ afforded the pure product $6 a(270 \mathrm{mg}, 1.32 \mathrm{mmol}, 66 \%)$ as a colorless solid.

\section{2,2,2-Trifluoro-1-(3-methoxyphenyl)ethan-1-one (6b)}<smiles>COc1cccc(C(=O)C(F)(F)F)c1</smiles>

Following TP4, precooled solutions of ethyl trifluoroacetate (7) (1.12 M, 1.2 equiv, $0.40 \mathrm{mmol})$ and (3-methoxyphenyl)magnesium bromide ( $0.93 \mathrm{M}, 1.0$ equiv, $0.33 \mathrm{mmol})$, prepared via TP1, were mixed in continuous flow (flowrate $A=10 \mathrm{~mL} \cdot \mathrm{min}^{-1}, V_{0}{ }^{R}=20 \mathrm{~mL}$, residence time: $t=1$ $\min , \mathrm{T}=-5^{\circ} \mathrm{C}$ ). Thereafter, the reaction mixture was quenched with $\mathrm{NH}_{4} \mathrm{Cl}$. After workup, the crude product was purified via column chromatography (isohexane:ethyl acetate $=9.8: 0.2$ ) to give 6 b (201.0 mg, $0.98 \mathrm{mmol}, 65 \%)$ as a yellow oil.

${ }^{1} \mathrm{H}-\mathrm{NMR}\left(400 \mathrm{MHz}, \mathrm{CDCl}_{3}\right): \delta / \mathrm{ppm}=7.68(\mathrm{dq}, J=7.8,1.4 \mathrm{~Hz}, 1 \mathrm{H}), 7.59(\mathrm{t}, J=2.2 \mathrm{~Hz}, 1 \mathrm{H})$, $7.48(\mathrm{t}, J=8.0 \mathrm{~Hz}, 1 \mathrm{H}), 7.31-7.23(\mathrm{~m}, 1 \mathrm{H}), 3.90(\mathrm{~s}, 3 \mathrm{H})$.

${ }^{13} \mathrm{C}-N M R\left(101 \mathrm{MHz}, \mathrm{CDCl}_{3}\right.$ ): $\delta / \mathrm{ppm}=180.4$ (q, $J=35.0 \mathrm{~Hz}$ ), 160.0, 131.1, 130.1, 122.7 (q, $J=2.7 \mathrm{~Hz}), 122.3,116.6(\mathrm{~d}, J=291.3 \mathrm{~Hz}), 114.0(\mathrm{q}, J=1.8 \mathrm{~Hz}), 55.5$.

The spectra matched those of the literature. ${ }^{3}$

\section{2,2,2-Trifluoro-1-(2-methoxyphenyl)ethan-1-one (6c)}<smiles>COc1ccccc1C(=O)C(F)(F)F</smiles>

Following TP4, precooled solutions of ethyl trifluoroacetate (7) (1.20 M, 1.2 equiv, $0.40 \mathrm{mmol}$ ) and (2-methoxyphenyl)magnesium bromide (1.00 M, 1.0 equiv, $0.33 \mathrm{mmol})$, prepared via TP1, were mixed in continuous flow (flowrate $A=5 \mathrm{~mL} \cdot \mathrm{min}^{-1}$, Vol ${ }^{R}=20 \mathrm{~mL}$, residence time: $t=2$ min , $\mathrm{T}=-5^{\circ} \mathrm{C}$ ). Thereafter, the reaction mixture was quenched with $\mathrm{NH}_{4} \mathrm{Cl}$. After workup, the crude product was purified via column chromatography (isohexane:ethyl acetate $=9.5: 0.5$ ) to give 6 c (51.0 mg, $0.25 \mathrm{mmol}, 75 \%)$ as a colorless oil. 
${ }^{1} \mathrm{H}-\mathrm{NMR}\left(400 \mathrm{MHz}, \mathrm{CDCl}_{3}\right): \delta / \mathrm{ppm}=7.67(\mathrm{~d}, J=7.8,1.9,0.6 \mathrm{~Hz}, 1 \mathrm{H}), 7.61-7.56(\mathrm{~m}, 1 \mathrm{H})$, $7.07-7.01(\mathrm{~m}, 2 \mathrm{H}), 3.91(\mathrm{~s}, 3 \mathrm{H})$.

${ }^{13} \mathrm{C}-N M R\left(101 \mathrm{MHz}, \mathbf{C D C l}_{3}\right): \delta / \mathrm{ppm}=183.0(\mathrm{q}, J=36.6 \mathrm{~Hz}), 159.8,135.9,131.3,121.7$, 120.7, 116.2 (q, $J=291.0 \mathrm{~Hz}$ ), 112.1, 55.9.

${ }^{19} \mathrm{~F}-\mathrm{NMR}$ (377 MHz, $\left.\mathrm{CDCl}_{3}\right)$ : $\delta / \mathrm{ppm}=-74.16$.

IR (Diamond-ATR, neat): $\tilde{v} / \mathrm{cm}^{-1}=2949,2845,2355,1709,1600,1489,1278,1145$, 1114, 1020, 930, 753, 657.

MS (El, $70 \mathrm{eV}): m / z(\%)=204$ (10), 135 (100), 92 (11), 77 (16).

HRMS (EI): $m / z$ calc. for $\left[\mathrm{C}_{9} \mathrm{H}_{7} \mathrm{~F}_{3} \mathrm{O}_{2}\right]$ : 204.0398; found 204.0392 .

The spectra matched those of the literature. ${ }^{3}$

\section{1-(Benzo[d][1,3]dioxol-5-yl)-2,2,2-trifluoroethan-1-one (6d)}<smiles>O=C(c1ccc2c(c1)OCO2)C(F)(F)F</smiles>

Following TP4, precooled solutions of ethyl trifluoroacetate (7) $(0.60 \mathrm{M}, 1.2$ equiv, $0.45 \mathrm{mmol})$ and benzo[d][1,3]dioxol-5-ylmagnesium bromide $(0.50 \mathrm{M}, 1.0$ equiv, $0.37 \mathrm{mmol})$, prepared via TP1, were mixed in continuous flow (flowrate $A=5 \mathrm{~mL} \cdot \mathrm{min}^{-1}, \mathrm{Vol}^{\mathrm{R}}=20 \mathrm{~mL}$, residence time: $\mathrm{t}$ $=2 \mathrm{~min}, \mathrm{~T}=-5^{\circ} \mathrm{C}$ ). Thereafter, the reaction mixture was quenched with $\mathrm{NH}_{4} \mathrm{Cl}$. After workup, the crude product was purified via column chromatography (isohexane:ethyl acetate $=9.7: 0.3$ ) to give $6 \mathbf{d}(50.0 \mathrm{mg}, 0.23 \mathrm{mmol}, 62 \%)$ as a colorless oil.

${ }^{1} \mathrm{H}-\mathrm{NMR}\left(400 \mathrm{MHz}, \mathrm{CDCl}_{3}\right): \delta / \mathrm{ppm}=7.72-7.66(\mathrm{~m}, 1 \mathrm{H}), 7.47(\mathrm{~s}, 1 \mathrm{H}), 6.91(\mathrm{~d}, J=8.3 \mathrm{~Hz}$, $1 \mathrm{H}), 6.10(\mathrm{~s}, 2 \mathrm{H})$.

${ }^{13} \mathrm{C}-N M R\left(101 \mathrm{MHz}, \mathrm{CDCl}_{3}\right): \delta / \mathrm{ppm}=178.6(\mathrm{q}, J=34.8 \mathrm{~Hz}), 154.0,148.6,127.6,124.4$, 116.81 (q, $J=291.2 \mathrm{~Hz}$ ), 109.2, 108.5, 102.5 .

The spectra matched those of the literature. ${ }^{5}$

\footnotetext{
${ }^{5}$ C. B. Kelly, M. A. Mercadante, T. H. Hamlin, M. H. Fletcher, N. E. Leadbeater, J. Org. Chem. 2012, 77, 8131-
} 8141. 


\section{2,2,2-Trifluoro-1-(6-methoxynaphthalen-2-yl)ethan-1-one (6e)}<smiles>COc1ccc2cc(C(=O)C(F)(F)F)ccc2c1</smiles>

Following TP4, precooled solutions of ethyl trifluoroacetate (7) (1.20 M, 1.2 equiv, $0.48 \mathrm{mmol}$ ) and (6-methoxynaphthalen-2-yl)magnesium (1.00 M, 1.0 equiv, $0.40 \mathrm{mmol})$, prepared via TP1, were mixed in continuous flow (flowrate $A=5 \mathrm{~mL} \cdot \mathrm{min}^{-1}$, Vol ${ }^{R}=20 \mathrm{~mL}$, residence time: $\mathrm{t}$ $=2 \mathrm{~min}, \mathrm{~T}=-5^{\circ} \mathrm{C}$ ). Thereafter, the reaction mixture was quenched with $\mathrm{NH}_{4} \mathrm{Cl}$. After workup, the crude product was purified via column chromatography (isohexane:ethyl acetate $=9.7: 0.3$ ) to give $6 \mathrm{e}(67.0 \mathrm{mg}, 0.27 \mathrm{mmol}, 69 \%)$ as a white solid.

${ }^{1} \mathrm{H}-\mathrm{NMR}\left(400 \mathrm{MHz}, \mathrm{CDCl}_{3}\right): \delta / \mathrm{ppm}=8.53(\mathrm{t}, J=1.9 \mathrm{~Hz}, 1 \mathrm{H}), 8.04$ (ddd, $J=8.8,1.9,0.8 \mathrm{~Hz}$, $1 \mathrm{H}), 7.89(\mathrm{dd}, J=8.9,0.7 \mathrm{~Hz}, 1 \mathrm{H}), 7.83-7.80(\mathrm{~m}, 1 \mathrm{H}), 7.25(\mathrm{dd}, J=9.1,2.6 \mathrm{~Hz}, 1 \mathrm{H}), 7.17$ (d, $J=2.5 \mathrm{~Hz}, 1 \mathrm{H}), 3.97(\mathrm{~s}, 3 \mathrm{H})$.

${ }^{13} \mathrm{C}-N M R\left(101 \mathrm{MHz}, \mathrm{CDCl}_{3}\right): \delta / \mathrm{ppm}=180.1(\mathrm{q}, J=34.6 \mathrm{~Hz}), 161.1,138.5,133.0,131.9$, 127.7, 127.5, 125.1, 125.1, 120.4, 117.0 (q, $J=291.5 \mathrm{~Hz}$ ), 105.9, 55.5.

${ }^{19} \mathrm{~F}-\mathrm{NMR}\left(377 \mathrm{MHz}, \mathrm{CDCl}_{3}\right): \delta / \mathrm{ppm}=-70.6$.

IR (Diamond-ATR, neat): $\tilde{v} / \mathrm{cm}^{-1}=3072,3015,2940,1697,1618,1481,14001266$, 1194, 1140, 1028, 900, 749.

MS (El, $70 \mathrm{eV}): m / z(\%)=254$ (35), 186 (13), 185 (100), 157 (38), 142 (25), 114 (16).

HRMS (EI): $m / z$ calc. for $\left[\mathrm{C}_{13} \mathrm{H}_{9} \mathrm{~F}_{3} \mathrm{O}_{2}\right]: 254.0555$; found 254.0584 .

M.p. $\left({ }^{\circ} \mathrm{C}\right): 69-70$.

\section{1-(3,5-Di-tert-butylphenyl)-2,2,2-trifluoroethan-1-one (6f)}<smiles>CC(C)(C)c1cc(C(=O)C(F)(F)F)cc(C(C)(C)C)c1</smiles>

Following TP4, precooled solutions of ethyl trifluoroacetate (7) $(0.89 \mathrm{M}, 1.2$ equiv, $1.78 \mathrm{mmol})$ and (3,5-di-tert-butylphenyl)magnesium bromide ( $0.74 \mathrm{M}, 1.0$ equiv, $1.48 \mathrm{mmol})$, prepared via 
TP1, were mixed in continuous flow (flowrate $A=5 \mathrm{~mL} \cdot \mathrm{min}^{-1}, \mathrm{Vol}^{\mathrm{R}}=20 \mathrm{~mL}$, residence time: $\mathrm{t}$ $=2 \mathrm{~min}, \mathrm{~T}=-5^{\circ} \mathrm{C}$ ). Thereafter, the reaction mixture was quenched with $\mathrm{NH}_{4} \mathrm{Cl}$. After workup, the crude product was purified via column chromatography (pentane $+1 \%$ triethylamine) to give $6 f(272 \mathrm{mg}, 0.95 \mathrm{mmol}, 64 \%)$ as a colorless oil.

${ }^{1} \mathrm{H}-\mathrm{NMR}\left(400 \mathrm{MHz}, \mathrm{CDCl}_{3}\right): \delta / \mathrm{ppm}=7.95(\mathrm{dd}, J=1.9,1.1 \mathrm{~Hz}, 2 \mathrm{H}), 7.81(\mathrm{t}, J=1.9 \mathrm{~Hz}, 1 \mathrm{H}$ ), $1.38(\mathrm{~s}, 18 \mathrm{H})$.

${ }^{13} \mathrm{C}-N M R\left(101 \mathrm{MHz}, \mathrm{CDCl}_{3}\right): \delta / \mathrm{ppm}=181.0$ (q, $\left.J=34.3 \mathrm{~Hz}\right), 151.9,130.0,129.6,124.4$, 116.9 (q, $J=291.7 \mathrm{~Hz}$ ), 35.1, 31.2.

${ }^{19} \mathrm{~F}-\mathrm{NMR}\left(377 \mathrm{MHz}, \mathrm{CDCl}_{3}\right): \delta / \mathrm{ppm}=-71.0$.

IR (Diamond-ATR, neat): $\tilde{v} / \mathrm{cm}^{-1}=2965,2356,1718,1594,1478,1366,1200,1178$, 1140, 996, 839, 709.

MS (El, $70 \mathrm{eV}): m / z(\%)=272(16), 271(100), 243(21)$.

HRMS (EI): $m / z$ calc. for $\left[\mathrm{C}_{16} \mathrm{H}_{21} \mathrm{~F}_{3} \mathrm{O}\right]: 286.1544$; found 286.1537 .

1-(Benzo[b]thiophen-2-yl)-2,2,2-trifluoroethan-1-one (6g)

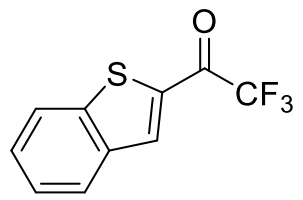

Following TP4, precooled solutions of ethyl trifluoroacetate (7) (0.6 M, 1.2 equiv, $0.60 \mathrm{mmol}$ ) and benzo[b]thiophen-2-ylmagnesium bromide $(0.50 \mathrm{M}, 1.0$ equiv, $0.50 \mathrm{mmol})$, prepared via TP1, were mixed in continuous flow (flowrate $A=5 \mathrm{~mL} \cdot \mathrm{min}^{-1}$, Vol ${ }^{R}=20 \mathrm{~mL}$, residence time: $\mathrm{t}$ $\left.=2 \min , \mathrm{T}=-5^{\circ} \mathrm{C}\right)$. Thereafter, the reaction mixture was quenched with $\mathrm{NH}_{4} \mathrm{Cl}$. After workup, the crude product was purified via column chromatography (isohexane:ethyl acetate $=9.9: 0.1$ ) to give $6 \mathrm{~g}$ (170 $\mathrm{mg}, 0.74 \mathrm{mmol}, 74 \%)$ as a yellow solid.

${ }^{1} \mathrm{H}-\mathrm{NMR}\left(400 \mathrm{MHz}, \mathrm{CDCl}_{3}\right): \delta / \mathrm{ppm}=8.24(\mathrm{dt}, J=2.4,1.2 \mathrm{~Hz}, 1 \mathrm{H}), 7.98(\mathrm{dt}, J=8.1,1.1 \mathrm{~Hz}$, 1H), 7.92 (dq, $J=8.3,0.9 \mathrm{~Hz}, 1 \mathrm{H}$ ), 7.57 (ddd, $J=8.3,7.1,1.3 \mathrm{~Hz}, 1 \mathrm{H}$ ), 7.48 (ddd, $J=8.1,7.1$, $1.1 \mathrm{~Hz}, 1 \mathrm{H})$.

${ }^{13} \mathrm{C}-\mathrm{NMR}\left(101 \mathrm{MHz}, \mathrm{CDCl}_{3}\right): \delta / \mathrm{ppm}=175.3(\mathrm{q}, J=37.0 \mathrm{~Hz}), 143.6,138.8,135.6,134.3$, 129.2, 127.1, 125.8, 122.9, 116.4 (q, $J=290.4 \mathrm{~Hz}$ ).

${ }^{19} \mathrm{~F}-\mathrm{NMR}$ (377 MHz, $\mathrm{CDCl}_{3}$ ): $\delta / \mathrm{ppm}=-71.8$.

IR (Diamond-ATR, neat): $\tilde{v} / \mathrm{cm}^{-1}=1682,1595,1508,1345,1250,1225,1189,1139,882$, 842, 760, 744, 718.

MS (El, $70 \mathrm{eV}): m / z(\%)=230$ (38), $162(10), 161$ (100), 133 (24), 89 (23).

HRMS (EI): $m / z$ calc. for [ $\left.\mathrm{C}_{9} \mathrm{H}_{5} \mathrm{~F}_{3} \mathrm{OS}\right]: 230.0006$; found 230.0013. 
M.p. $\left({ }^{\circ} \mathrm{C}\right): 50-51$.

\section{2,2,2-Trifluoro-1-(1-methyl-1H-indol-5-yl)ethan-1-one (6h)}<smiles>Cn1ccc2cc(C(=O)C(F)(F)F)ccc21</smiles>

Following TP4, precooled solutions of ethyl trifluoroacetate (7) $(0.27 \mathrm{M}, 1.2$ equiv, $0.27 \mathrm{mmol})$ and (1-methyl-1H-indol-5-yl)magnesium bromide ( $0.22 \mathrm{M}, 1.0$ equiv, $0.22 \mathrm{mmol}$ ), prepared via TP1, were mixed in continuous flow (flowrate $A=5 \mathrm{~mL} \cdot \mathrm{min}^{-1}, \mathrm{Vol}^{\mathrm{R}}=20 \mathrm{~mL}$, residence time: $\mathrm{t}$ $=2 \mathrm{~min}, \mathrm{~T}=-5^{\circ} \mathrm{C}$ ). Thereafter, the reaction mixture was quenched with $\mathrm{NH}_{4} \mathrm{Cl}$. After workup, the crude product was purified via column chromatography (isohexane:ethyl acetate $=9.0: 1.0$ )

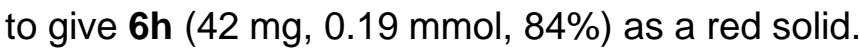

${ }^{1} \mathrm{H}-\mathrm{NMR}\left(400 \mathrm{MHz}, \mathrm{CDCl}_{3}\right): \delta / \mathrm{ppm}=8.47-8.41(\mathrm{~m}, 1 \mathrm{H}), 7.99(\mathrm{~m}, 1 \mathrm{H}), 7.42(\mathrm{dt}, J=8.8,0.8$ $\mathrm{Hz}, 1 \mathrm{H}), 7.19$ (d, J=3.2 Hz, 1H), 6.69 (dd, $J=3.2,0.9 \mathrm{~Hz}, 1 \mathrm{H}), 3.87$ (s, 3H).

${ }^{13} \mathrm{C}-N M R\left(101 \mathrm{MHz}, \mathbf{C D C l}_{3}\right): \delta / \mathrm{ppm}=180.4(\mathrm{q}, J=33.8 \mathrm{~Hz}), 140.2,131.3,128.1,125.8$, 123.3, 121.8, 117.3 (q, $J=291.8 \mathrm{~Hz}), 109.9,103.9,33.1$.

${ }^{19} \mathrm{~F}-\mathrm{NMR}\left(377 \mathrm{MHz}, \mathrm{CDCl}_{3}\right): \delta / \mathrm{ppm}=-71.0$.

IR (Diamond-ATR, neat): $\tilde{v} / \mathrm{cm}^{-1}=2950,2360,1696,1606,1218,1192,1138,1099,962$, 752, 718.

MS (El, $70 \mathrm{eV}): m / z(\%)=227$ (47), 159 (11), 158 (100), 130 (45), 128 (21), 103 (15), 77 (12). HRMS (EI): $m / z$ calc. for $\left[\mathrm{C}_{11} \mathrm{H}_{8} \mathrm{~F}_{3} \mathrm{NO}\right]: 227.0558$; found 227.0550 .

M.p. $\left({ }^{\circ} \mathrm{C}\right): 55-56$.

\section{Ethyl 4-(2,2,2-trifluoroacetyl)benzoate (6i)}<smiles>CCOC(=O)c1ccc(C(=O)C(F)(F)F)cc1</smiles>

Following TP4, precooled solutions of ethyl trifluoroacetate (7) (0.6 M, 1.2 equiv, $1.50 \mathrm{mmol})$ and (4-(ethoxycarbonyl)phenyl)magnesium chloride ( $0.50 \mathrm{M}, 1.0$ equiv, $1.25 \mathrm{mmol}$ ), prepared 
via TP2 $\left(-30{ }^{\circ} \mathrm{C}, 30 \mathrm{~min}\right.$ ), were mixed in continuous flow (flowrate $A=5 \mathrm{~mL} \cdot \mathrm{min}^{-1}, \mathrm{Vol}^{\mathrm{R}}=$ $20 \mathrm{~mL}$, residence time: $\mathrm{t}=2 \mathrm{~min}, \mathrm{~T}=15^{\circ} \mathrm{C}$ ). Thereafter, the reaction mixture was quenched with $\mathrm{NH}_{4} \mathrm{Cl}$. After workup, the crude product was purified via column chromatography (pentane/ $\left.\mathrm{Et}_{2} \mathrm{O}=7.0: 3: 0\right)$ to give $6 \mathbf{i}(218 \mathrm{mg}, 0.89 \mathrm{mmol}, 71 \%$ ) as a colorless oil.

${ }^{1} \mathrm{H}-\mathrm{NMR}\left(400 \mathrm{MHz}, \mathrm{CDCl}_{3}\right): \delta / \mathrm{ppm}=8.22-8.18(\mathrm{~m}, 2 \mathrm{H}), 8.13(\mathrm{dq}, J=7.7,1.0 \mathrm{~Hz}, 2 \mathrm{H}), 4.43$ (q, $J=7.2 \mathrm{~Hz}, 2 \mathrm{H}), 1.42(\mathrm{t}, J=7.1 \mathrm{~Hz}, 3 \mathrm{H}$ ).

${ }^{13} \mathrm{C}-N M R\left(101 \mathrm{MHz}, \mathbf{C D C l}_{3}\right): \delta / \mathrm{ppm}=180.2$ (q, $\left.J=35.7 \mathrm{~Hz}\right), 165.1,136.3,132.9,130.1$, 130.0 (d, $J=7.3 \mathrm{~Hz}), 116.5$ (q, $J=291.0 \mathrm{~Hz}), 61.8,14.2$.

${ }^{19} \mathrm{~F}-\mathrm{NMR}\left(377 \mathrm{MHz}, \mathrm{CDCl}_{3}\right): \delta / \mathrm{ppm}=-71.7$.

IR (Diamond-ATR, neat): $\tilde{v} / \mathrm{cm}^{-1}=2985,2358,1721,1276,1205,1181,1145,1106$, 1021, 941, 730, 696.

MS (El, $70 \mathrm{eV}): m / z(\%)=246$ (25), 201 (64), 178 (11), 177 (100), 173 (14), 149 (77), 123 (16), 104 (11).

HRMS (EI): $m / z$ calc. for $\left[\mathrm{C}_{11} \mathrm{H}_{9} \mathrm{~F}_{3} \mathrm{O}_{3}\right]: 246.0504$; found 246.0497 .

(3-Methoxyphenyl)(pyridin-2-yl)methanone (10a)

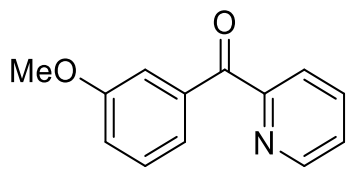

Following TP4, solutions of ethyl 2-picolinate 9a (0.6 M, 1.2 equiv, $0.60 \mathrm{mmol})$ and (3-methoxyphenyl)magnesium bromide $(0.50 \mathrm{M}, 1.0$ equiv, $0.50 \mathrm{mmol})$, prepared via TP1, were mixed in continuous flow (flowrate $A=5 \mathrm{~mL} \cdot \mathrm{min}^{-1}, \mathrm{Vol}^{\mathrm{R}}=20 \mathrm{~mL}$, residence time: $\mathrm{t}=2$ min , $\mathrm{T}=25^{\circ} \mathrm{C}$ ). Thereafter, the reaction mixture was quenched with $\mathrm{NH}_{4} \mathrm{Cl}$. After workup, the crude product was purified via column chromatography (isohexane:ethyl acetate $=9.9: 0.1$ ) to give 10a (170 mg, $0.74 \mathrm{mmol}, 75 \%$ ) as a pink liquid.

${ }^{1} \mathrm{H}-N M R\left(400 \mathrm{MHz}, \mathrm{CDCl}_{3}\right): \delta / \mathrm{ppm}=8.69(\mathrm{ddd}, J=4.8,1.8,1.0 \mathrm{~Hz}, 1 \mathrm{H}), 7.99(\mathrm{dt}, J=7.8$, $1.2 \mathrm{~Hz}, 1 \mathrm{H}$ ), 7.86 (td, $J=7.7,1.7 \mathrm{~Hz}, 1 \mathrm{H}$ ), $7.63-7.55(\mathrm{~m}, 2 \mathrm{H}), 7.45$ (ddd, $J=7.6,4.8,1.3 \mathrm{~Hz}$, $1 \mathrm{H}$ ), 7.36 (t, $J=7.9 \mathrm{~Hz}, 1 \mathrm{H}$ ), 7.12 (ddd, $J=8.3,2.7,1.1 \mathrm{~Hz}, 1 \mathrm{H}$ ).

${ }^{13} \mathrm{C}-N M R\left(101 \mathrm{MHz}, \mathrm{CDCl}_{3}\right): \delta / \mathrm{ppm}=193.7,159.4,155.1,148.6,137.5,137.1,129.2,126.2$, 124.6, 123.9, 119.5, 115.1, 55.5. 
IR (Diamond-ATR, neat): $\tilde{v} / \mathrm{cm}^{-1}=2835,1661,1595,1578,1484,1431,1304,1282,1248$, $1141,1041,994,954,829,745,706$

MS (El, $70 \mathrm{eV}): m / z(\%)=213$ (31), 212 (65), 198 (17), 186 (11), 185 (84), 184 (100), 182 (21), 170 (22), 156 (12), 155 (16), 154 (14), 135 (67), 107 (31), 77 (26)

HRMS (EI): $m / z$ calc. for $\left[\mathrm{C}_{13} \mathrm{H}_{11} \mathrm{NO}_{2}\right]: 213.0790$; found 213.0782 .

\section{4-Picolinoylbenzonitrile (10b)}<smiles>N#Cc1ccc(C(=O)c2ccccn2)cc1</smiles>

Following TP4, solutions of ethyl 2-picolinate 9a $(0.23 \mathrm{M}, 1.2$ equiv, $0.23 \mathrm{mmol})$ and (4cyanophenyl)magnesium chloride $(0.19 \mathrm{M}, 1.0$ equiv, $0.19 \mathrm{mmol})$, prepared via TP2 $\left(-30^{\circ} \mathrm{C}\right.$, $30 \mathrm{~min}$ ), were mixed in continuous flow (flowrate $A=1 \mathrm{~mL} \cdot \mathrm{min}^{-1}, \mathrm{Vol}^{\mathrm{R}}=20 \mathrm{~mL}$, residence time: $\mathrm{t}=10 \mathrm{~min}, \mathrm{~T}=25^{\circ} \mathrm{C}$ ). Thereafter, the reaction mixture was quenched with $\mathrm{NH}_{4} \mathrm{Cl}$. After workup, the crude product was purified via column chromatography (isohexane:ethyl acetate $=9.0: 1.0)$ to give $10 \mathrm{~b}(30 \mathrm{mg}, 0.15 \mathrm{mmol}, 77 \%)$ as a white solid.

${ }^{1} \mathrm{H}-\mathrm{NMR}\left(400 \mathrm{MHz}, \mathrm{CDCl}_{3}\right): \delta / \mathrm{ppm}=8.72(\mathrm{ddd}, J=4.8,1.7,0.9 \mathrm{~Hz}, 1 \mathrm{H}), 8.21-8.25(\mathrm{~m}$, 2H), $8.12(\mathrm{dt}, J=7.9,1.1 \mathrm{~Hz}, 1 \mathrm{H}), 7.91(\mathrm{td}, J=7.8,1.8 \mathrm{~Hz}, 1 \mathrm{H}), 7.83-7.75(\mathrm{~m}, 2 \mathrm{H}), 7.58$ (ddd, $J=7.6,4.8,1.3 \mathrm{~Hz}, 1 \mathrm{H}$ ).

${ }^{13} \mathrm{C}-\mathrm{NMR}\left(101 \mathrm{MHz}, \mathrm{CDCl}_{3}\right): \delta / \mathrm{ppm}=192.2,153.8,148.7,139.9,137.4,131.9,131.4,127.0$, 124.8, 118.2, 115.8 .

IR (Diamond-ATR, neat): $\tilde{v} / \mathrm{cm}^{-1}=2924,1730,1669,1584,1437,1407,1309,1285,1244$, 1157, 996, 938, 856, 804, 749, 705, 680.

MS (El, 70 eV): m/z (\%) = 207 (56), 181 (13), 180 (100), 179 (70), 130 (20), 130 (23).

HRMS (EI): $m / z$ calc. for $\left[\mathrm{C}_{13} \mathrm{H}_{7} \mathrm{~N}_{2} \mathrm{O}\right]: 207.0564$; found $207.0551\left(\mathrm{M}^{+}-\mathrm{H}\right)$.

M.p. ( $\left.{ }^{\circ} \mathrm{C}\right): 116-117$.

3-Phenyl-1-(pyridin-2-yl)propan-1-one (10c) 
<smiles>O=C(CCc1ccccc1)c1ccccn1</smiles>

Following TP4, solutions of ethyl 2-picolinate $9 \mathrm{a}(0.27 \mathrm{M}, 1.5$ equiv, $0.27 \mathrm{mmol})$ and phenethylmagnesium bromide $(0.18 \mathrm{M}, 1.0$ equiv, $0.18 \mathrm{mmol})$, prepared via TP1, were mixed in continuous flow (flowrate $A=1 \mathrm{~mL} \cdot \mathrm{min}^{-1}, \mathrm{Vol}^{\mathrm{R}}=20 \mathrm{~mL}$, residence time: $\mathrm{t}=10 \mathrm{~min}, \mathrm{~T}=$ $0{ }^{\circ} \mathrm{C}$ ). Thereafter, the reaction mixture was quenched with $\mathrm{NH}_{4} \mathrm{Cl}$. After workup, the crude product was purified via column chromatography (isohexane:ethyl acetate $=8.0: 2.0$ ) to give 10c (24 mg, $0.12 \mathrm{mmol}, 63 \%)$ as a colorless oil.

${ }^{1} \mathrm{H}-\mathrm{NMR}\left(400 \mathrm{MHz}, \mathrm{CDCl}_{3}\right): \delta / \mathrm{ppm}=8.67$ (ddd, $\left.J=4.7,1.8,0.9 \mathrm{~Hz}, 1 \mathrm{H}\right), 8.05(\mathrm{dt}, J=7.9$, $1.1 \mathrm{~Hz}, 1 \mathrm{H}), 7.83(\mathrm{td}, J=7.7,1.7 \mathrm{~Hz}, 1 \mathrm{H}), 7.46$ (ddd, $J=7.5,4.7,1.3 \mathrm{~Hz}, 1 \mathrm{H}), 7.28(\mathrm{~d}, J=4.4$ $\mathrm{Hz}, 4 \mathrm{H}), 7.23-7.17(\mathrm{~m}, 1 \mathrm{H}), 3.58(\mathrm{dd}, J=8.3,7.2 \mathrm{~Hz}, 2 \mathrm{H}), 3.08(\mathrm{dd}, J=8.2,7.2 \mathrm{~Hz}, 2 \mathrm{H}$ ).

${ }^{13} \mathrm{C}-\mathrm{NMR}\left(101 \mathrm{MHz}, \mathrm{CDCl}_{3}\right): \delta / \mathrm{ppm}=201.0,153.3,149.0,141.4,136.9,128.5,128.4,127.1$, 126.0, 121.8, 39.4, 29.9.

IR (Diamond-ATR, neat): $\tilde{v} / \mathrm{cm}^{-1}=3028,2926,1697,1583,1454,1437,1363,1305,1212$, 995, 980.

MS (El, $70 \mathrm{eV}): m / z(\%)=211(17), 184(10), 183$ (73), 183 (12), $182(89), 91(14), 79(100)$, 78 (18).

HRMS (EI): $m / z$ calc. for $\left[\mathrm{C}_{14} \mathrm{H}_{13} \mathrm{NO}\right]: 211.0997$; found 211.0990 .

\section{(2-(Dimethylamino)phenyl)(pyrazin-2-yl)methanone (10d)}<smiles>COc1ccccc1C(=O)c1cnccn1</smiles>

Following TP4, solutions of methyl pyrazine-2-carboxylate $9 \mathrm{~b}(0.32 \mathrm{M}, 1.5$ equiv, $0.32 \mathrm{mmol})$ and (2-(dimethylamino)phenyl)magnesium bromide $(0.21 \mathrm{M}, 1.0$ equiv, $0.21 \mathrm{mmol})$, prepared via TP1, were mixed in continuous flow (flowrate $A=1 \mathrm{~mL} \cdot \mathrm{min}^{-1}, \mathrm{Vol}^{R}=20 \mathrm{~mL}$, residence time: $\mathrm{t}=10 \mathrm{~min}, \mathrm{~T}=25^{\circ} \mathrm{C}$ ). Thereafter, the reaction mixture was quenched with $\mathrm{NH}_{4} \mathrm{Cl}$. After workup, the crude product was purified via column chromatography (isohexane:ethyl acetate $=7.0: 3.0)$ to give $10 \mathrm{~d}(30 \mathrm{mg}, 0.13 \mathrm{mmol}, 64 \%)$ as an orange oil. 
${ }^{1} \mathrm{H}-\mathrm{NMR}\left(400 \mathrm{MHz}, \mathrm{CDCl}_{3}\right): \delta / \mathrm{ppm}=9.04(\mathrm{~d}, J=1.5 \mathrm{~Hz}, 1 \mathrm{H}), 8.68(\mathrm{~d}, J=2.5 \mathrm{~Hz}, 1 \mathrm{H}), 8.62$ $(\mathrm{dd}, J=2.5,1.5 \mathrm{~Hz}, 1 \mathrm{H}), 7.52-7.44(\mathrm{~m}, 2 \mathrm{H}), 7.06$ (dt, $J=8.2,0.8 \mathrm{~Hz}, 1 \mathrm{H}), 7.01$ (td, $J=7.4$, $1.0 \mathrm{~Hz}, 1 \mathrm{H}), 2.62(\mathrm{~s}, 6 \mathrm{H})$.

${ }^{13} \mathrm{C}-\mathrm{NMR}\left(101 \mathrm{MHz}, \mathrm{CDCl}_{3}\right): \delta / \mathrm{ppm}=195.6,153.0,150.7,146.4,145.2,143.6,133.0,131.2$, 128.7, 120.3, 117.5, 43.9 .

IR (Diamond-ATR, neat): $\tilde{v} / \mathrm{cm}^{-1}=2927,2865,2360,1663,1596,1496,1454,1430$, 1306, 1268, 1156, 1050, 1017, 953, 933, 921, 752.

MS (El, $70 \mathrm{eV}): m / z(\%)=210$ (35), 209 (100), 195 (14), 156 (13), 148 (14), 130 (14), 120 (13), 118 (11), 104 (22), 94 (10), 91 (19), 77 (21).

HRMS (EI): $m / z$ calc. for $\left[\mathrm{C}_{13} \mathrm{H}_{13} \mathrm{~N}_{3} \mathrm{O}\right]: 227.1059$; found 227.1053.

(3-Chlorophenyl)(pyrazin-2-yl)methanone (10e)

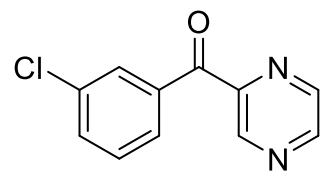

Following TP4, solutions of methyl pyrazine-2-carboxylate $9 \mathrm{~b}(0.9 \mathrm{M}, 1.2$ equiv, $1.08 \mathrm{mmol})$ and (3-chlorophenyl)magnesium bromide $(0.75 \mathrm{M}, 1.0$ equiv, $0.75 \mathrm{mmol})$, prepared via TP1, were mixed in continuous flow (flowrate $A=10 \mathrm{~mL} \cdot \mathrm{min}^{-1}$, Vol ${ }^{R}=20 \mathrm{~mL}$, residence time: $\mathrm{t}=2$ min , $\mathrm{T}=25^{\circ} \mathrm{C}$ ). Thereafter, the reaction mixture was quenched with $\mathrm{NH}_{4} \mathrm{Cl}$. After workup, the crude product was purified via column chromatography (isohexane:ethyl acetate $=(8.0: 2.0)$ to give $10 \mathrm{e}(210 \mathrm{mg}, 0.13 \mathrm{mmol}, 51 \%)$ as an orange oil.

${ }^{1} \mathrm{H}-\mathrm{NMR}\left(400 \mathrm{MHz}, \mathrm{CDCl}_{3}\right): \delta / \mathrm{ppm}=9.27(\mathrm{~d}, J=1.5 \mathrm{~Hz}, 1 \mathrm{H}), 8.80(\mathrm{~d}, J=2.5 \mathrm{~Hz}, 1 \mathrm{H}), 8.69$ (dd, $J=2.5,1.5 \mathrm{~Hz}, 1 \mathrm{H}), 8.10(\mathrm{t}, J=1.9 \mathrm{~Hz}, 1 \mathrm{H}), 8.05-7.93(\mathrm{~m}, 1 \mathrm{H}), 7.69-7.55(\mathrm{~m}, 1 \mathrm{H})$, $7.45(\mathrm{t}, J=7.9 \mathrm{~Hz}, 1 \mathrm{H})$.

${ }^{13} \mathrm{C}-\mathrm{NMR}\left(101 \mathrm{MHz}, \mathbf{C D C l}_{3}\right): \delta / \mathrm{ppm}=190.8,149.2,147.2,146.2,142.9,137.0,134.5,133.4$, 130.8, 129.7, 129.0 . 
IR (Diamond-ATR, neat): $\tilde{v} / \mathrm{cm}^{-1}=3068,1662,1566,1421,1401,1293,1267,1154,1016$, 949, 762, 703.

MS (El, $70 \mathrm{eV}): m / z(\%)=218$ (15), 183 (25), 141 (32), 139 (100), 111 (18), 75 (23).

HRMS (EI): $m / z$ calc. for $\left[\mathrm{C}_{11} \mathrm{H}_{7} \mathrm{CIN}_{2} \mathrm{O}\right]: 218.0247$; found 218.0238 .

4-(Pyrazine-2-carbonyl)benzonitrile (10f)<smiles>N#Cc1ccc(C(=O)c2cnccn2)cc1</smiles>

Following TP4, solutions of pyrazine-2-carboxylate $9 \mathrm{~b}(0.27 \mathrm{M}, 1.5$ equiv, $0.27 \mathrm{mmol})$ and (4cyanophenyl)magnesium chloride $(0.19 \mathrm{M}, 1.0$ equiv, $0.19 \mathrm{mmol})$, prepared via TP2 $\left(-30^{\circ} \mathrm{C}\right.$, $30 \mathrm{~min}$ ), were mixed in continuous flow (flowrate $A=1 \mathrm{~mL} \cdot \mathrm{min}^{-1}, \mathrm{Vol}^{R}=20 \mathrm{~mL}$, residence time: $\mathrm{t}=10 \mathrm{~min}, \mathrm{~T}=25^{\circ} \mathrm{C}$ ). Thereafter, the reaction mixture was quenched with $\mathrm{NH}_{4} \mathrm{Cl}$. After workup, the crude product was purified via column chromatography (isohexane:ethyl acetate $=9.0: 1.0)$ to give $10 f(21.0 \mathrm{mg}, 0.10 \mathrm{mmol}, 55 \%)$ as a yellow solid.

${ }^{1} \mathrm{H}-\mathrm{NMR}\left(400 \mathrm{MHz}, \mathrm{CDCl}_{3}\right): \delta / \mathrm{ppm}=9.34(\mathrm{~d}, J=1.5 \mathrm{~Hz}, 1 \mathrm{H}), 8.84(\mathrm{~d}, J=2.5 \mathrm{~Hz}, 1 \mathrm{H}), 8.69$ (dd, $J=2.5,1.5 \mathrm{~Hz}, 1 \mathrm{H}), 8.23-8.21(\mathrm{~m}, 2 \mathrm{H}), 7.82-7.80(\mathrm{~m}, 2 \mathrm{H})$.

${ }^{13} \mathrm{C}-\mathrm{NMR}\left(101 \mathrm{MHz}, \mathrm{CDCl}_{3}\right): \delta / \mathrm{ppm}=190.8,148.6,147.6,146.3,142.9,138.9,132.1,131.3$, 118.0, 116.5 .

IR (Diamond-ATR, neat): $\tilde{v} / \mathrm{cm}^{-1}=2064,2360,2234,1673,1406,1302,1153,1019$, 934, 770.

MS (El, $70 \mathrm{eV}): m / z(\%)=209$ (27), 181 (41), 130 (35), 130 (100), 102 (10).

HRMS (EI): $m / z$ calc. for $\left[\mathrm{C}_{12} \mathrm{H}_{7} \mathrm{~N}_{3} \mathrm{O}\right]: 209.0589$; found 209.0582.

M.p. $\left({ }^{\circ} \mathrm{C}\right): 127-128$.

(2,6-Dimethoxyphenyl)(pyrimidin-2-yl)methanone (10g)<smiles>COc1cccc(OC)c1C(=O)c1ncccn1</smiles> 
Following TP4, solutions of methyl pyrimidine-2-carboxylate $9 \mathrm{c}(0.27 \mathrm{M}, 1.5$ equiv, $0.27 \mathrm{mmol})$ and (2,6-dimethoxyphenyl)magnesium bromide (0.18 M, 1.0 equiv, $0.18 \mathrm{mmol})$, prepared via TP1, were mixed in continuous flow (flowrate $A=1 \mathrm{~mL} \cdot \mathrm{min}^{-1}, \mathrm{Vol}^{\mathrm{R}}=20 \mathrm{~mL}$, residence time: $\mathrm{t}$ $=40 \mathrm{~min}, \mathrm{~T}=25^{\circ} \mathrm{C}$ ). Thereafter, the reaction mixture was quenched with $\mathrm{NH}_{4} \mathrm{Cl}$. After workup, the crude product was purified via column chromatography (isohexane:ethyl acetate $=8.0: 2.0$ ) to give $10 \mathrm{~g}(51 \mathrm{mg}, 0.21 \mathrm{mmol}, 60 \%)$ as a yellow solid.

${ }^{1} \mathrm{H}-\mathrm{NMR}\left(400 \mathrm{MHz}, \mathrm{CDCl}_{3}\right): \delta / \mathrm{ppm}=8.91(\mathrm{~d}, J=4.8 \mathrm{~Hz}, 2 \mathrm{H}), 7.62-7.33(\mathrm{~m}, 2 \mathrm{H}), 6.62(\mathrm{~d}$, $J=8.4 \mathrm{~Hz}, 2 \mathrm{H}), 3.69(\mathrm{~s}, 6 \mathrm{H})$.

${ }^{13} \mathrm{C}-N M R\left(101 \mathrm{MHz}, \mathrm{CDCl}_{3}\right): \delta / \mathrm{ppm}=192.6,161.9,158.8,157.6,132.2,122.3,117.3,104.2$, 56.0 .

IR (Diamond-ATR, neat): $\tilde{v} / \mathrm{cm}^{-1}=2845,1705,1590,1473,1434,1407,1306,1286,1250$, $1104,943,784,759,740,702$

MS (El, $70 \mathrm{eV}): m / z(\%)=244$ (), 213 (46), 165 (100), 150 (25), 122 (15), 107 (23)

HRMS (EI): $m / z$ calc. for $\left[\mathrm{C}_{13} \mathrm{H}_{12} \mathrm{~N}_{2} \mathrm{O}_{3}\right]$ : 244.0848; found 244.0844 .

M.p. ( $\left.{ }^{\circ} \mathrm{C}\right): 127-128$.

Ethyl 2-(2-methoxyphenyl)-2-oxoacetate (12a)<smiles>CCOC(=O)c1ccccc1OC</smiles>

Following TP4, solutions of diethyl oxalate (11) (0.53 M, 1.2 equiv, $1.32 \mathrm{mmol})$ and (2methoxyphenyl)magnesium bromide $(0.44 \mathrm{M}, 1.0$ equiv, $1.10 \mathrm{mmol})$, prepared via TP1, were mixed in continuous flow (flowrate $A=1 \mathrm{~mL} \cdot \mathrm{min}^{-1}, \mathrm{Vol}^{\mathrm{R}}=20 \mathrm{~mL}$, residence time: $\mathrm{t}=10 \mathrm{~min}$, $\mathrm{T}=25^{\circ} \mathrm{C}$ ). Thereafter, the reaction mixture was quenched with $\mathrm{NH}_{4} \mathrm{Cl}$. After workup, the crude product was purified via column chromatography (isohexane:ethyl acetate $=9.0: 1.0$ ) to give 12a (190 mg, $0.91 \mathrm{mmol}, 83 \%$ ) as a yellow oil.

${ }^{1} \mathrm{H}-\mathrm{NMR}\left(400 \mathrm{MHz}, \mathrm{CDCl}_{3}\right.$ ): $\delta / \mathrm{ppm}=7.80(\mathrm{ddd}, J=7.8,1.9,1.1 \mathrm{~Hz}, 1 \mathrm{H}), 7.52$ (dddt, $J=8.0$, 7.3, 1.6, $0.8 \mathrm{~Hz}, 1 \mathrm{H}), 7.00$ (tt, $J=7.3,0.9 \mathrm{~Hz}, 1 \mathrm{H}), 6.93(\mathrm{~d}, J=8.4 \mathrm{~Hz}, 1 \mathrm{H}), 4.35-4.30(\mathrm{~m}$, 2H), 3.80 (t, $J=0.8 \mathrm{~Hz}, 3 \mathrm{H}), 1.32$ (tt, $J=7.2,0.8 \mathrm{~Hz}, 3 \mathrm{H})$.

${ }^{13} \mathrm{C}-\mathrm{NMR}\left(101 \mathrm{MHz}, \mathrm{CDCl}_{3}\right): \delta / \mathrm{ppm}=186.6,165.3,160.3,136.4,130.6,122.7,121.3,112.1$, $61.8,56.0,14.1$. 
The spectra matched those of the literature. ${ }^{6}$

\section{Ethyl 2-(benzo[d][1,3]dioxol-5-yl)-2-oxoacetate (12b)}<smiles>CCOC(=O)c1ccc2c(c1)OCO2</smiles>

Following TP4, solutions of diethyl oxalate (11) $(0.60 \mathrm{M}, 1.2$ equiv, $0.60 \mathrm{mmol})$ and benzo[d][1,3]dioxol-5-ylmagnesium bromide $(0.50 \mathrm{M}, 1.0$ equiv, $0.50 \mathrm{mmol})$, prepared via TP1, were mixed in continuous flow (flowrate $A=1 \mathrm{~mL} \cdot \mathrm{min}^{-1}, \mathrm{Vol}^{\mathrm{R}}=20 \mathrm{~mL}$, residence time: $\mathrm{t}$ $=10 \mathrm{~min}, \mathrm{~T}=25^{\circ} \mathrm{C}$ ). Thereafter, the reaction mixture was quenched with $\mathrm{NH}_{4} \mathrm{Cl}$. After workup, the crude product was purified via column chromatography (isohexane:ethyl acetate $=8.5: 1.5$ ) to give $\mathbf{1 2 b}(70.0 \mathrm{mg}, 0.31 \mathrm{mmol}, 63 \%)$ as an orange oil.

${ }^{1} \mathrm{H}-\mathrm{NMR}\left(400 \mathrm{MHz}, \mathrm{CDCl}_{3}\right): \delta / \mathrm{ppm}=7.61(\mathrm{dt}, J=8.2,1.8 \mathrm{~Hz}, 1 \mathrm{H}), 7.47(\mathrm{t}, J=1.8 \mathrm{~Hz}, 1 \mathrm{H})$, 6.89 (dd, $J=8.2,1.8 \mathrm{~Hz}, 1 \mathrm{H}$ ), 6.08 (d, $J=1.8 \mathrm{~Hz}, 2 \mathrm{H}$ ), 4.42 (qd, $J=7.1,1.7 \mathrm{~Hz}, 2 \mathrm{H}$ ), 1.41 (td, $J=7.2,1.8 \mathrm{~Hz}, 3 \mathrm{H})$.

${ }^{13} \mathrm{C}-\mathrm{NMR}\left(101 \mathrm{MHz}, \mathrm{CDCl}_{3}\right): \delta / \mathrm{ppm}=184.6,164.0,153.5,148.5,127.9,127.2,108.7,108.3$, 102.2, 62.3, 14.1 .

The spectra matched those of the literature. ${ }^{4}$

\section{Ethyl 2-(3-fluoro-4-methoxyphenyl)-2-oxoacetate (12c)}<smiles>CCOC(=O)c1ccc(OC)c(F)c1</smiles>

Following TP4, solutions of diethyl oxalate (11) (0.32 M, 1.4 equiv, $0.32 \mathrm{mmol}$ ) and (3-fluoro4-methoxyphenyl)magnesium bromide $(0.23 \mathrm{M}, 1.0$ equiv, $0.23 \mathrm{mmol})$, prepared via TP1, were mixed in continuous flow (flowrate $A=1 \mathrm{~mL} \cdot \mathrm{min}^{-1}, V_{0}{ }^{R}=20 \mathrm{~mL}$, residence time: $t=10$

${ }^{6}$ Y. Kumar, Y. Jaiswal, A. Kumar, J. Org. Chem. 2016, 81,12247-12257. 
min , $\mathrm{T}=25^{\circ} \mathrm{C}$ ). Thereafter, the reaction mixture was quenched with $\mathrm{NH}_{4} \mathrm{Cl}$. After workup, the crude product was purified via column chromatography (isohexane:ethyl acetate $=9.0: 1.0$ ) to give 12c (36.0 mg, $0.16 \mathrm{mmol}, 69 \%)$ as a yellow oil.

${ }^{1} \mathrm{H}-\mathrm{NMR}\left(400 \mathrm{MHz}, \mathrm{CDCl}_{3}\right): \delta / \mathrm{ppm}=7.84(\mathrm{ddd}, J=8.6,2.1,1.1 \mathrm{~Hz}, 1 \mathrm{H}), 7.78(\mathrm{dd}, J=11.5$, $2.1 \mathrm{~Hz}, 1 \mathrm{H}$ ), 7.03 (t, $J=8.3 \mathrm{~Hz}, 1 \mathrm{H}), 4.43$ (q, $J=7.1 \mathrm{~Hz}, 2 \mathrm{H}), 3.97(\mathrm{~s}, 3 \mathrm{H}), 1.41$ (t, $J=7.1 \mathrm{~Hz}$, $3 \mathrm{H})$.

${ }^{13} \mathrm{C}-N M R\left(101 \mathrm{MHz}, \mathrm{CDCl}_{3}\right): \delta / \mathrm{ppm}=183.9,163.4,153.4(\mathrm{~d}, J=32.0 \mathrm{~Hz}), 150.8,128.3$, 125.7, 117.2 (d, $J=19.5 \mathrm{~Hz}), 112.6,62.4,56.4,14.1$.

IR (Diamond-ATR, neat): $\tilde{v} / \mathrm{cm}^{-1}=2984,2941,2360,1731,1679,1608,1516,1439$, $1284,1253,1221,1161,1118,1015,896,762$.

MS (El, $70 \mathrm{eV}): m / z(\%)=153(100)$.

HRMS (EI): $m / z$ calc. for $\left[\mathrm{C}_{11} \mathrm{H}_{11} \mathrm{FO}_{4}\right]: 226.0641$; found 226.0643 .

Ethyl 2-(2-fluoro-[1,1'-biphenyl]-4-yl)-2-oxoacetate (12d)<smiles>CCOC(=O)c1ccc(-c2ccccc2)c(F)c1</smiles>

Following TP4, solutions of diethyl oxalate (11) $(0.30 \mathrm{M}, 1.5$ equiv, $0.30 \mathrm{mmol})$ and (2-fluoro[1,1'-biphenyl]-4-yl)magnesium bromide $(0.20 \mathrm{M}, 1.0$ equiv, $0.20 \mathrm{mmol})$, prepared via TP1, were mixed in continuous flow (flowrate $A=1 \mathrm{~mL} \cdot \mathrm{min}^{-1}$, Vol ${ }^{R}=20 \mathrm{~mL}$, residence time: $t=10$ $\min , \mathrm{T}=25^{\circ} \mathrm{C}$ ). Thereafter, the reaction mixture was quenched with $\mathrm{NH}_{4} \mathrm{Cl}$. After workup, the crude product was purified via column chromatography (isohexane:ethyl acetate $=9.0: 1.0$ ) to give $12 \mathrm{~d}$ (45.0 mg, $0.17 \mathrm{mmol}, 79 \%)$ as a colorless oil.

${ }^{1} \mathrm{H}-\mathrm{NMR}\left(400 \mathrm{MHz}, \mathrm{CDCl}_{3}\right): \delta / \mathrm{ppm}=7.92-7.82(\mathrm{~m}, 2 \mathrm{H}), 7.63-7.56(\mathrm{~m}, 3 \mathrm{H}), 7.52-7.38$ (m, 3H), 4.48 (q, J=7.2 Hz, 2H), 1.45 (t, $J=7.2 \mathrm{~Hz}, 3 \mathrm{H})$.

${ }^{13} \mathrm{C}-N M R\left(101 \mathrm{MHz}, \mathrm{CDCl}_{3}\right): \delta / \mathrm{ppm}=184.6,163.1,160.9,158.4,135.8(\mathrm{~d}, J=13.7 \mathrm{~Hz})$, $134.3(\mathrm{~d}, J=1.5 \mathrm{~Hz}), 133.1$ (d, $J=7.0 \mathrm{~Hz}), 131.3$ (d, $J=3.5 \mathrm{~Hz}), 129.1$ (d, $J=3.2 \mathrm{~Hz}), 128.7$, $126.3(\mathrm{~d}, J=3.5 \mathrm{~Hz}), 117.5(\mathrm{~d}, J=24.9 \mathrm{~Hz}), 62.6,14.1$. 
IR (Diamond-ATR, neat): $\tilde{v} / \mathrm{cm}^{-1}=3452,2984,1731,1687,1612,1408,1300,1248,1215$, 1154, 1132, 1121, 1025, 1010, 892, 765, 720, 696.

MS (El, $70 \mathrm{eV}): m / z(\%)=200(11), 199$ (100), 171 (18), 170 (47).

HRMS (EI): $m / z$ calc. for $\left[\mathrm{C}_{16} \mathrm{H}_{13} \mathrm{O}_{3} \mathrm{~F}\right]: 272.0849$; found 272.0837.

Ethyl 2-oxo-2-(4-(trifluoromethyl)phenyl)acetate (12e)<smiles>CCOC(=O)c1ccc(C(F)(F)F)cc1</smiles>

Following TP4, solutions of diethyl oxalate (11) $(0.30 \mathrm{M}, 1.2$ equiv, $0.30 \mathrm{mmol})$ and (4(trifluoromethyl)phenyl)magnesium bromide (0.24 M, 1.0 equiv, $0.24 \mathrm{mmol})$, prepared via TP1, were mixed in continuous flow (flowrate $A=1 \mathrm{~mL} \cdot \mathrm{min}^{-1}$, Vol ${ }^{R}=20 \mathrm{~mL}$, residence time: $\mathrm{t}=10$ $\min , \mathrm{T}=25^{\circ} \mathrm{C}$ ). Thereafter, the reaction mixture was quenched with $\mathrm{NH}_{4} \mathrm{Cl}$. After workup, the crude product was purified via column chromatography (isohexane:ethyl acetate $=8.0: 2.0$ ) to give $12 \mathrm{e}(41.0 \mathrm{mg}, 0.17 \mathrm{mmol}, 71 \%)$ as a colorless oil.

${ }^{1} \mathrm{H}-\mathrm{NMR}\left(400 \mathrm{MHz}, \mathrm{CDCl}_{3}\right): \delta / \mathrm{ppm}=8.17-8.15(\mathrm{~m}, 2 \mathrm{H}), 7.78(\mathrm{~d}, J=8.2 \mathrm{~Hz}, 2 \mathrm{H}), 4.47$ (q, $J=7.1 \mathrm{~Hz}, 2 \mathrm{H}), 1.43$ (t, $J=7.2 \mathrm{~Hz}, 3 \mathrm{H}$ ).

${ }^{13} \mathrm{C}-N M R\left(101 \mathrm{MHz}, \mathbf{C D C l}_{3}\right): \delta / \mathrm{ppm}=\delta 185.0,162.8,135.9(\mathrm{q}, J=32.9 \mathrm{~Hz}), 135.3$ (d, $J=$ $1.3 \mathrm{~Hz}), 130.4,125.9,62.8,14.1$.

${ }^{19} \mathrm{~F}-\mathrm{NMR}\left(377 \mathrm{MHz}, \mathrm{CDCl}_{3}\right): \delta / \mathrm{ppm}=-63.4$.

IR (Diamond-ATR, neat): $\tilde{v} / \mathrm{cm}^{-1}=2987,1735,1697,1412,1323,1203,1166,1125$, 1112, 1065, 1013, 981, 847.

MS (EI, $70 \mathrm{eV}): m / z(\%)=173(100), 145(35)$.

HRMS (EI): $m / z$ calc. for $\left[\mathrm{C}_{11} \mathrm{H}_{9} \mathrm{O}_{3} \mathrm{~F}_{3}\right]: 246.0504$; found 246.0489 .

(2-Methoxyphenyl)(phenyl)methanone (16a)<smiles>COc1ccccc1C(=O)c1ccccc1</smiles> 
Following TP4, solutions of alkoxide $14 a(0.60 \mathrm{M}, 1.2$ equiv, $0.60 \mathrm{mmol})$, prepared via TP3, and (2-methoxyphenyl)magnesium bromide $(0.48 \mathrm{M}, 1.0$ equiv, $0.48 \mathrm{mmol})$, prepared via TP1, were mixed in continuous flow (flowrate $A=1 \mathrm{~mL} \cdot \mathrm{min}^{-1}, \mathrm{Vol}^{\mathrm{R}}=20 \mathrm{~mL}$, residence time: $\mathrm{t}=10$ min , $\mathrm{T}=25^{\circ} \mathrm{C}$ ). Thereafter, the reaction mixture was quenched with $\mathrm{NH}_{4} \mathrm{Cl}$. After workup, the crude product was purified via column chromatography (isohexane:ethyl acetate $=9.0: 1.0$ ) to give 16 a $(80.0 \mathrm{mg}, 0.38 \mathrm{mmol}, 78 \%)$ as an orange oil.

${ }^{1} \mathrm{H}-\mathrm{NMR}\left(400 \mathrm{MHz}, \mathrm{CDCl}_{3}\right): \delta / \mathrm{ppm}=7.84-7.80(\mathrm{~m}, 2 \mathrm{H}), 7.58-7.53(\mathrm{~m}, 1 \mathrm{H}), 7.50-7.41$ (m, 3H), 7.36 (dd, $J=7.5,1.8 \mathrm{~Hz}, 1 \mathrm{H}), 7.07-6.98(\mathrm{~m}, 2 \mathrm{H}), 3.73(\mathrm{~s}, 3 \mathrm{H})$.

${ }^{13} \mathrm{C}-N M R\left(101 \mathrm{MHz}, \mathbf{C D C l}_{3}\right): \delta / \mathrm{ppm}=196.5,157.4,137.8,132.9,131.9,129.8,129.6,128.9$, 128.2, 120.5, 111.5, 55.6.

The spectra matched those of the literature. ${ }^{7}$

\section{Benzo[d][1,3]dioxol-5-yl(phenyl)methanone (16b)}

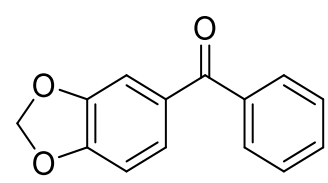

Following TP4, solutions of alkoxide $14 a(0.27 \mathrm{M}, 1.2$ equiv, $0.27 \mathrm{mmol})$, prepared via TP3, and benzo[d][1,3]dioxol-5-ylmagnesium bromide ( $0.23 \mathrm{M}, 1.0$ equiv, $0.23 \mathrm{mmol})$, prepared via TP1, were mixed in continuous flow (flowrate $A=1 \mathrm{~mL} \cdot \mathrm{min}^{-1}, \mathrm{Vol}^{\mathrm{R}}=20 \mathrm{~mL}$, residence time: $\mathrm{t}$ $=10 \mathrm{~min}, \mathrm{~T}=25^{\circ} \mathrm{C}$ ). Thereafter, the reaction mixture was quenched with $\mathrm{NH}_{4} \mathrm{Cl}$. After workup, the crude product was purified via column chromatography (isohexane:ethyl acetate $=9.0: 1.0$ ) to give $16 \mathrm{~b}$ (32.0 $\mathrm{mg}, 0.14 \mathrm{mmol}, 62 \%$ ) as a yellow oil.

${ }^{1} \mathrm{H}-\mathrm{NMR}\left(400 \mathrm{MHz}, \mathrm{CDCl}_{3}\right): \delta / \mathrm{ppm}=7.76-7.73(\mathrm{~m}, 2 \mathrm{H}), 7.56(\mathrm{ddt}, J=8.7,7.0,1.3 \mathrm{~Hz}, 1 \mathrm{H})$, $7.49-7.45(\mathrm{~m}, 2 \mathrm{H}), 7.39-7.35(\mathrm{~m}, 2 \mathrm{H}), 6.86(\mathrm{~d}, J=8.0 \mathrm{~Hz}, 1 \mathrm{H}), 6.06(\mathrm{~s}, 2 \mathrm{H})$.

${ }^{13} \mathrm{C}-N M R\left(101 \mathrm{MHz}, \mathrm{CDCl}_{3}\right): \delta / \mathrm{ppm}=195.2,151.5,148.0,138.1,132.0,131.9,129.7,128.2$, 126.9, 109.9, 107.7, 101.9.

\footnotetext{
${ }^{7}$ H. Neumann, A. Brennführer, M- Beller, Chem. Eur. J., 2008, 14, 3645-3652.
} 
The spectra matched those of the literature. ${ }^{8}$

(4-Chlorophenyl)(3,5-dimethoxyphenyl)methanone (16c)<smiles>COc1cc(OC)cc(C(=O)c2ccc(Cl)cc2)c1</smiles>

Following TP4, solutions of alkoxide $14 \mathrm{~b}(0.84 \mathrm{M}, 1.2$ equiv, $0.84 \mathrm{mmol})$, prepared via TP3, and (3,5-dimethoxyphenyl)magnesium bromide ( $0.70 \mathrm{M}, 1.0$ equiv, $0.70 \mathrm{mmol})$, prepared via TP1, were mixed in continuous flow (flowrate $A=1 \mathrm{~mL} \cdot \mathrm{min}^{-1}, \mathrm{Vol}^{\mathrm{R}}=20 \mathrm{~mL}$, residence time: $\mathrm{t}$ $=10 \mathrm{~min}, \mathrm{~T}=25^{\circ} \mathrm{C}$ ). Thereafter, the reaction mixture was quenched with $\mathrm{NH}_{4} \mathrm{Cl}$. After workup, the crude product was purified via column chromatography (isohexane:ethyl acetate $=9.5: 0.5$ ) to give $16 \mathrm{c}$ (120 $\mathrm{mg}, 0.43 \mathrm{mmol}, 62 \%$ ) as a colorless oil.

${ }^{1} \mathrm{H}-\mathrm{NMR}\left(400 \mathrm{MHz}, \mathrm{CDCl}_{3}\right): \delta / \mathrm{ppm}=7.76(\mathrm{~d}, J=8.5 \mathrm{~Hz}, 2 \mathrm{H}), 7.45(\mathrm{~d}, J=8.5 \mathrm{~Hz}, 2 \mathrm{H}), 6.88$ (d, $J=2.3 \mathrm{~Hz}, 2 \mathrm{H}), 6.68$ (t, $J=2.3 \mathrm{~Hz}, 1 \mathrm{H}), 3.83(\mathrm{~s}, 6 \mathrm{H})$.

${ }^{13} \mathrm{C}-\mathrm{NMR}\left(101 \mathrm{MHz}, \mathrm{CDCl}_{3}\right): \delta / \mathrm{ppm}=195.1,160.6,139.1,138.9,135.8,131.4,128.6,107.8$, 104.9, 55.6.

IR (Diamond-ATR, neat): $\tilde{v} / \mathrm{cm}^{-1}=2938,2839,2362,1659,1587,1455,1425,1352,1324$, 1300, 1205, 1156, 1090, 1065, 990, 842, 812, 759.

MS (El, $70 \mathrm{eV}): m / z(\%)=278$ (32), 277 (16), 276 (100), 241 (26), 226 (15), 165 (85), 140 (28), 139 (13), 139 (85), 137 (31), 122 (15), 111 (12).

HRMS (EI): $m / z$ calc. for $\left[\mathrm{C}_{15} \mathrm{H}_{13} \mathrm{O}_{3} \mathrm{Cl}\right]: 276.0553$; found 276.0545 .

(4-(Tert-butyl)phenyl)(4-chlorophenyl)methanone (16d)<smiles>CC(C)(C)c1ccc(C(=O)c2ccc(Cl)cc2)cc1</smiles>

${ }^{8}$ A. M. Echavarren, J. K. Stille, J. Am. Chem. Soc. 1988, 110, 1557-1565. 
Following TP4, solutions of alkoxide $14 \mathrm{~b}(0.60 \mathrm{M}, 1.2$ equiv, $0.60 \mathrm{mmol})$, prepared via TP3, and (4-(tert-butyl)phenyl)magnesium bromide $(0.45 \mathrm{M}, 1.0$ equiv, $0.45 \mathrm{mmol})$, prepared via TP1, were mixed in continuous flow (flowrate $A=1 \mathrm{~mL} \cdot \mathrm{min}^{-1}, \mathrm{Vol}^{\mathrm{R}}=20 \mathrm{~mL}$, residence time: $\mathrm{t}$ $=10 \mathrm{~min}, \mathrm{~T}=25^{\circ} \mathrm{C}$ ). Thereafter, the reaction mixture was quenched with $\mathrm{NH}_{4} \mathrm{Cl}$. After workup, the crude product was purified via column chromatography (isohexane:ethyl acetate $=9.0: 1.0$ ) to give $\mathbf{1 6 d}$ (91.0 mg, $0.33 \mathrm{mmol}, 74 \%$ ) as a white solid.

${ }^{1} \mathrm{H}-\mathrm{NMR}\left(400 \mathrm{MHz}, \mathrm{CDCl}_{3}\right): \delta / \mathrm{ppm}=7.77-7.74(\mathrm{~m}, 2 \mathrm{H}), 7.74-7.72(\mathrm{~m}, 2 \mathrm{H}), 7.52-7.49$ $(\mathrm{m}, 2 \mathrm{H}), 7.47-7.44(\mathrm{~m}, 2 \mathrm{H}), 1.37(\mathrm{~d}, J=1.0 \mathrm{~Hz}, 9 \mathrm{H})$.

${ }^{13} \mathrm{C}-N M R\left(101 \mathrm{MHz}, \mathrm{CDCl}_{3}\right): \delta / \mathrm{ppm}=195.2,156.5,138.6,136.2,134.4,131.4,130.0,128.5$, 125.4, 35.1, 31.1.

The spectra matched those of the literature. ${ }^{9}$

\section{Benzo[d][1,3]dioxol-5-yl(3,4-difluorophenyl)methanone (16e)}<smiles>O=C(c1ccc(F)c(F)c1)c1ccc2c(c1)OCO2</smiles>

Following TP4, solutions of alkoxide $14 \mathrm{c}(0.27 \mathrm{M}, 1.2$ equiv, $0.27 \mathrm{mmol})$, prepared via TP3, and benzo[d][1,3]dioxol-5-ylmagnesium bromide ( $0.23 \mathrm{M}, 1.0$ equiv, $0.23 \mathrm{mmol})$, prepared via TP1, were mixed in continuous flow (flowrate $A=1 \mathrm{~mL} \cdot \mathrm{min}^{-1}, \mathrm{Vol}^{\mathrm{R}}=20 \mathrm{~mL}$, residence time: $\mathrm{t}$ $=10 \mathrm{~min}, \mathrm{~T}=25^{\circ} \mathrm{C}$ ). Thereafter, the reaction mixture was quenched with $\mathrm{NH}_{4} \mathrm{Cl}$. After workup, the crude product was purified via column chromatography (isohexane:ethyl acetate $=9.0: 1.0$ ) to give $16 \mathrm{e}$ ( $38.0 \mathrm{mg}, 0.14 \mathrm{mmol}, 63 \%$ ) as a white solid.

${ }^{1} \mathrm{H}-N M R\left(400 \mathrm{MHz}, \mathrm{CDCl}_{3}\right): \delta / \mathrm{ppm}=7.56$ (ddd, $\left.J=10.5,7.7,2.1 \mathrm{~Hz}, 1 \mathrm{H}\right), 7.53-7.47(\mathrm{~m}$, $1 \mathrm{H}), 7.28-7.25(\mathrm{~m}, 2 \mathrm{H}), 7.26-7.19(\mathrm{~m}, 1 \mathrm{H}), 6.82(\mathrm{dd}, J=7.8,0.6 \mathrm{~Hz}, 1 \mathrm{H}), 6.02(\mathrm{~s}, 2 \mathrm{H})$.

${ }^{13} \mathrm{C}-N M R\left(101 \mathrm{MHz}, \mathrm{CDCl}_{3}\right): \delta / \mathrm{ppm}=192.4,151.9,151.5(\mathrm{qd}, J=251.3,35.6,12.9 \mathrm{~Hz})$, 148.16, 134.94 (d, J = 3.9 Hz), 131.14, 126.72, 126.70 - 126.59 (m), 119.19, 117.12, 109.73, 107.86, 102.02.

IR (Diamond-ATR, neat): $\tilde{v} / \mathrm{cm}^{-1}=1656,1603,1515,1442,1305,1291,1249,1110$,

\footnotetext{
${ }^{9}$ H. Li, Y. Xu, E. Shi, W. Wei, X. Suo, X. Wan, Chem. Commun. 2011, 47, 7880-7882.
} 
1040, 904, 724.

MS (El, $70 \mathrm{eV}): m / z(\%)=262$ (41), 149 (100), 141 (48), 121 (18),

HRMS (EI): $m / z$ calc. for $\left[\mathrm{C}_{14} \mathrm{H}_{8} \mathrm{~F}_{2} \mathrm{O}_{3}\right]: 262.0442$; found 262.0435 .

M.p. $\left({ }^{\circ} \mathrm{C}\right):$ 77-78.

\section{(3,4-Difluorophenyl)(1-methyl-1H-indol-5-yl)methanone (16f)}

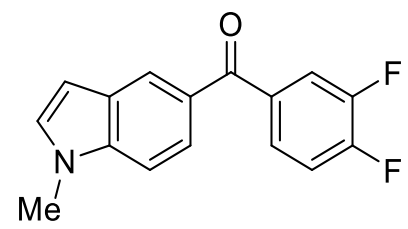

Following TP4, solutions of alkoxide $14 \mathrm{c}(0.33 \mathrm{M}, 1.5$ equiv, $0.33 \mathrm{mmol})$, prepared via TP3, and (1-methyl-1H-indol-5-yl)magnesium bromide ( $0.22 \mathrm{M}, 1.0$ equiv, $0.22 \mathrm{mmol})$, prepared via TP1, were mixed in continuous flow (flowrate $A=1 \mathrm{~mL} \cdot \mathrm{min}^{-1}$, Vol ${ }^{R}=20 \mathrm{~mL}$, residence time: $\mathrm{t}$ $=10 \mathrm{~min}, \mathrm{~T}=25^{\circ} \mathrm{C}$ ). Thereafter, the reaction mixture was quenched with $\mathrm{NH}_{4} \mathrm{Cl}$. After workup, the crude product was purified via column chromatography (isohexane:ethyl acetate $=9.0: 1.0$ ) to give $\mathbf{1 6 f}(47.0 \mathrm{mg}, 0.17 \mathrm{mmol}, 79 \%$ ) as a red solid.

${ }^{1} \mathrm{H}-\mathrm{NMR}\left(400 \mathrm{MHz}, \mathrm{CDCl}_{3}\right): \delta / \mathrm{ppm}=8.07(\mathrm{dd}, J=1.7,0.7 \mathrm{~Hz}, 1 \mathrm{H}), 7.77(\mathrm{dd}, J=8.6,1.7 \mathrm{~Hz}$, $1 \mathrm{H}$ ), $7.71-7.64(\mathrm{~m}, 1 \mathrm{H}), 7.59$ (dddd, $J=8.5,4.4,2.1,1.3 \mathrm{~Hz}, 1 \mathrm{H}), 7.40$ (dt, $J=8.7,0.8 \mathrm{~Hz}$, $1 \mathrm{H}), 7.31-7.23(\mathrm{~m}, 1 \mathrm{H}), 7.16(\mathrm{~d}, J=3.2 \mathrm{~Hz}, 1 \mathrm{H}), 6.60$ (dd, J=3.2, $0.9 \mathrm{~Hz}, 1 \mathrm{H}), 3.86(\mathrm{~s}, 3 \mathrm{H})$. ${ }^{13} \mathrm{C}-N M R\left(101 \mathrm{MHz}, \mathrm{CDCl}_{3}\right): \delta / \mathrm{ppm}=194.6,154.5-148.3(\mathrm{~m}), 139.1,136.0(\mathrm{t}, J=4.1 \mathrm{~Hz})$, 130.7, 128.4, 127.7, 126.8 (dd, $J=7.1,3.7 \mathrm{~Hz}$ ), 125.2, 123.6, 119.2 (dd, $J=18.0,1.5 \mathrm{~Hz}$ ), 117.0 (d, $J=17.7 \mathrm{~Hz}), 109.3,103.1,33.1$.

IR (Diamond-ATR, neat): $\tilde{v} / \mathrm{cm}^{-1}=2924,2360,1647,1600,1511,1422,1341,1313$, 1279, 1172, 1108, 1089,773, 742, 732.

MS (El, $70 \mathrm{eV}): m / z(\%)=272$ (10), 217 (64), 159 (10), 158 (100), 130 (25).

HRMS (EI): $m / z$ calc. for [ $\left.\mathrm{C}_{16} \mathrm{H}_{11} \mathrm{~F}_{2} \mathrm{NO}\right]: 271.0809$; found 271.0804 .

M.p. $\left({ }^{\circ} \mathrm{C}\right): 104-105$.

(4-Bromophenyl)(o-tolyl)methanone (16g) 
<smiles>Cc1ccccc1C(=O)c1ccc(Br)cc1</smiles>

Following TP4, solutions of alkoxide $14 \mathbf{d}(0.33 \mathrm{M}, 1.2$ equiv, $0.33 \mathrm{mmol})$, prepared via TP3, and o-tolylmagnesium bromide ( $0.27 \mathrm{M}, 1.0$ equiv, $0.27 \mathrm{mmol})$, prepared via TP1, were mixed in continuous flow (flowrate $A=1 \mathrm{~mL} \cdot \mathrm{min}^{-1}$, Vol ${ }^{R}=20 \mathrm{~mL}$, residence time: $\mathrm{t}=10 \mathrm{~min}, \mathrm{~T}=$ $25^{\circ} \mathrm{C}$ ). Thereafter, the reaction mixture was quenched with $\mathrm{NH}_{4} \mathrm{Cl}$. After workup, the crude product was purified via column chromatography (isohexane:ethyl acetate $=9.9: 0.1$ ) to give $\mathbf{1 6 g}(60.0 \mathrm{mg}, 0.22 \mathrm{mmol}, 81 \%)$ as a colorless oil.

${ }^{1} \mathrm{H}-\mathrm{NMR}\left(400 \mathrm{MHz}, \mathrm{CDCl}_{3}\right)$ : $\delta / \mathrm{ppm}=7.68-7.65(\mathrm{~m}, 2 \mathrm{H}), 7.61-7.59(\mathrm{~m}, 2 \mathrm{H}), 7.40$ (td, $\mathrm{J}=$ 7.3, $1.9 \mathrm{~Hz}, 1 \mathrm{H}), 7.31-7.25(\mathrm{~m}, 3 \mathrm{H}), 2.33(\mathrm{~s}, 3 \mathrm{H})$.

${ }^{13} \mathrm{C}-\mathrm{NMR}\left(101 \mathrm{MHz}, \mathrm{CDCl}_{3}\right): \delta / \mathrm{ppm}=197.5,138.0,136.8,136.5,131.8,131.6,131.2,130.5$, 128.5, 128.4, 125.3, 20.0.

IR (Diamond-ATR, neat): $\tilde{v} / \mathrm{cm}^{-1}=3063,2926,2362,1665,1584,1480,1454,1395,1265$, 1068, 1011, 924, 845, 743.

MS (El, $70 \mathrm{eV}): m / z(\%)=196$ (16), 195 (100), 194 (36), 177 (24), 165 (14), 91 (13).

HRMS (EI): $m / z$ calc. for $\left[\mathrm{C}_{14} \mathrm{H}_{10} \mathrm{OBr}\right]: 272.9921$; found $272.9909\left(\mathrm{M}^{+}-\mathrm{H}\right)$.

\section{4-(2-Methylbenzoyl)benzonitrile (16h)}

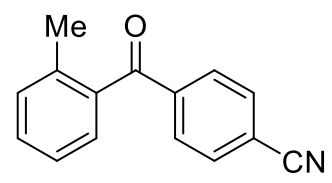

Following TP4, solutions of alkoxide $14 \mathrm{e}(0.33 \mathrm{M}, 1.2$ equiv, $0.33 \mathrm{mmol})$, prepared via TP3, and $o$-tolylmagnesium bromide $(0.27 \mathrm{M}, 1.0$ equiv, $0.27 \mathrm{mmol})$, prepared via TP1, were mixed in continuous flow (flowrate $A=1 \mathrm{~mL} \cdot \mathrm{min}^{-1}$, Vol ${ }^{R}=20 \mathrm{~mL}$, residence time: $\mathrm{t}=10 \mathrm{~min}, \mathrm{~T}=$ $25^{\circ} \mathrm{C}$ ). Thereafter, the reaction mixture was quenched with $\mathrm{NH}_{4} \mathrm{Cl}$. After workup, the crude product was purified via column chromatography (isohexane:ethyl acetate $=9.5: 0.5$ ) to give 16h (40.0 mg, $0.18 \mathrm{mmol}, 68 \%)$ as a colorless oil.

${ }^{1} \mathrm{H}-N M R\left(400 \mathrm{MHz}, \mathrm{CDCl}_{3}\right): \delta / \mathrm{ppm}=7.90-7.86(\mathrm{~m}, 2 \mathrm{H}), 7.79-7.74(\mathrm{~m}, 2 \mathrm{H}), 7.44$ (ddd, $\mathrm{J}$ $=7.6,6.3,2.4 \mathrm{~Hz}, 1 \mathrm{H}), 7.33(\mathrm{dq}, J=7.1,0.7 \mathrm{~Hz}, 1 \mathrm{H}), 7.29-7.26(\mathrm{~m}, 2 \mathrm{H}), 2.36(\mathrm{~d}, J=0.7 \mathrm{~Hz}$, $3 \mathrm{H})$.

${ }^{13} \mathrm{C}-\mathrm{NMR}\left(101 \mathrm{MHz}, \mathrm{CDCl}_{3}\right): \delta / \mathrm{ppm}=196.9,141.2,137.5,137.0,132.3,131.5,131.2,130.4$, 129.0, 125.5, 118.0, 116.2, 20.2. 
IR (Diamond-ATR, neat): $\tilde{v} / \mathrm{cm}^{-1}=2065,2937,1665,1405,1310,1294,1264,927,857$, 749.

MS (El, $70 \mathrm{eV}): m / z(\%)=221$ (26), 220 (100), 203 (11), 119 (10), 91 (15).

HRMS (EI): $m / z$ calc. for [ $\left.\mathrm{C}_{15} \mathrm{H}_{11} \mathrm{NO}\right]: 221.0841$; found 221.0835 .

(6-Chloropyridin-3-yl)(3-methoxyphenyl)methanone (16i)

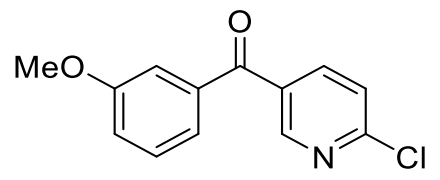

Following TP4, solutions of alkoxide $14 \mathrm{f}(0.35 \mathrm{M}, 1.5$ equiv, $0.35 \mathrm{mmol})$, prepared via TP3, and (3-methoxyphenyl)magnesium (0.23 M, 1.0 equiv, $0.23 \mathrm{mmol})$, prepared via TP1, were mixed in continuous flow (flowrate $A=1 \mathrm{~mL} \cdot \mathrm{min}^{-1}, \mathrm{Vol}^{\mathrm{R}}=20 \mathrm{~mL}$, residence time: $\mathrm{t}=10 \mathrm{~min}$, $\mathrm{T}=25^{\circ} \mathrm{C}$ ). Thereafter, the reaction mixture was quenched with $\mathrm{NH}_{4} \mathrm{Cl}$. After workup, the crude product was purified via column chromatography (isohexane:ethyl acetate $=9.0: 1.0$ ) to give $16 \mathbf{i}(37.0 \mathrm{mg}, 0.15 \mathrm{mmol}, 63 \%)$ as a colorless oil.

${ }^{1} \mathrm{H}-\mathrm{NMR}\left(400 \mathrm{MHz}, \mathrm{CDCl}_{3}\right.$ ): $\delta / \mathrm{ppm}=8.77$ (dd, $\left.J=2.4,0.8 \mathrm{~Hz}, 1 \mathrm{H}\right), 8.09$ (dd, $J=8.3,2.4 \mathrm{~Hz}$, $1 \mathrm{H}$ ), 7.48 (dd, $J=8.2,0.8 \mathrm{~Hz}, 1 \mathrm{H}$ ), 7.42 (ddd, $J=8.1,7.5,0.4 \mathrm{~Hz}, 1 \mathrm{H}), 7.35-7.28(\mathrm{~m}, 2 \mathrm{H}$ ), 7.18 (ddd, $J=8.2,2.7,1.0 \mathrm{~Hz}, 1 \mathrm{H}), 3.87$ (s, 3H).

${ }^{13} \mathrm{C}-\mathrm{NMR}\left(101 \mathrm{MHz}, \mathbf{C D C l}_{3}\right): \delta / \mathrm{ppm}=193.4,159.9,155.0,151.2,139.8,137.7,132.0,129.7$, 124.3, 122.7, 119.9, 114.1, 55.6.

IR (Diamond-ATR, neat): $\tilde{v} / \mathrm{cm}^{-1}=2964,2837,1662,1579,1485,1450,1427,1359,1280$, 1240, 1104, 759 .

MS (EI, $70 \mathrm{eV}): m / z(\%)=249$ (23), 247 (69), 246 (34), 218 (33), 216 (50), $212(61), 139$ (38), 135 (100), 111 (39), 107 (45), 77 (28).

HRMS (EI): $m / z$ calc. for $\left[\mathrm{C}_{13} \mathrm{H}_{10} \mathrm{O}_{2} \mathrm{NCl}\right]: 247.0400$; found 247.0394 . 
Condition Screening Results for the Reaction of p-Anisylmagnesium Bromide (2a) with Ethyl Trifluoroacetate (7)

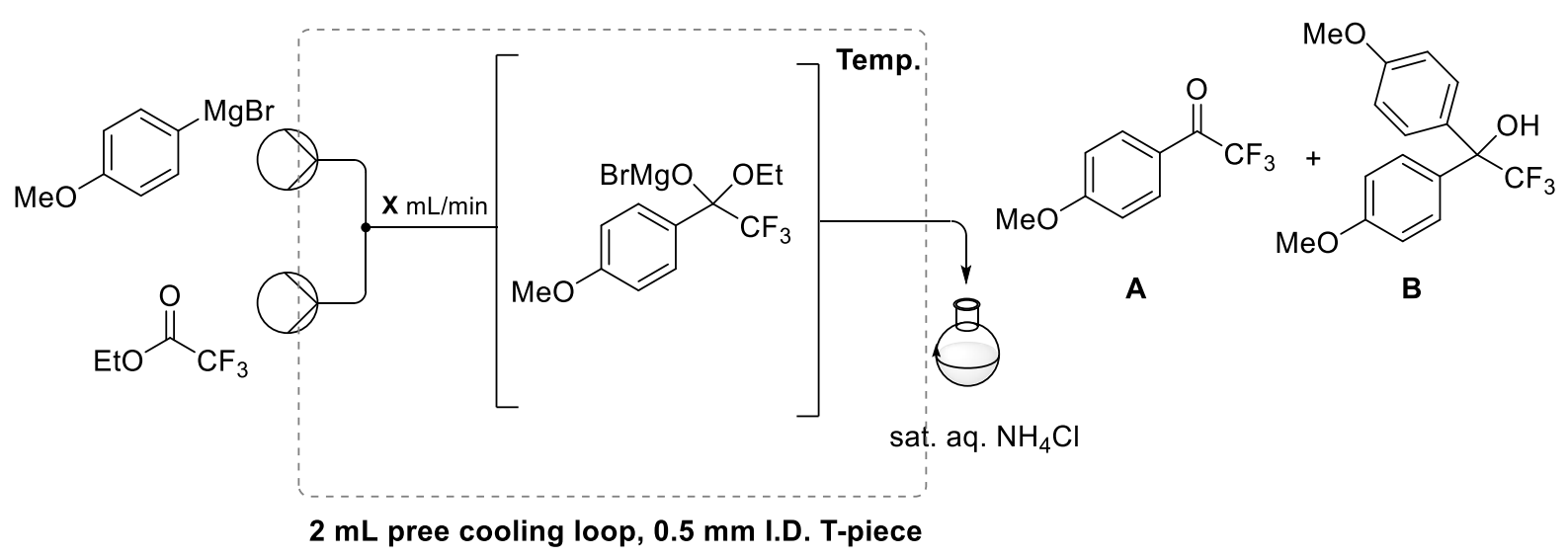

Graph 1. Temperature optimization for the reaction of $p$-anisylmagnesium bromide (2a) with ethyl trifluoroacetate (7).

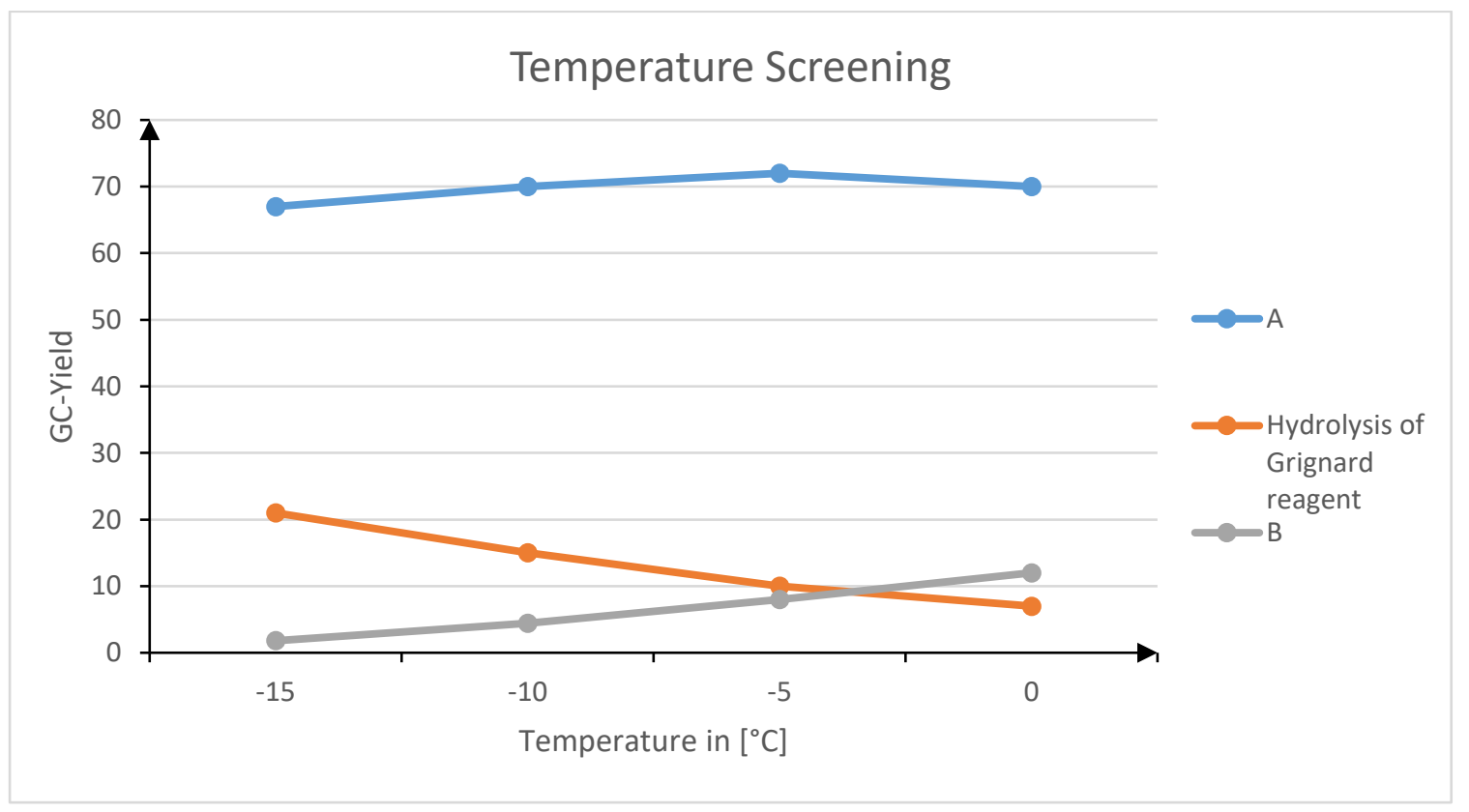

Temperature has a significant impact on the reaction selectivity towards ketone. Even variations of $5^{\circ} \mathrm{C}$ can impact conversion and selectivity. For this example $-5^{\circ} \mathrm{C}$ was found as optimum (Graph 1).

Residence time was optimized at $-5^{\circ} \mathrm{C}$. It was found, that the ketone formation is fast in the first minute and small improvements can be achieved with prolonging residence time to $2 \mathrm{~min}$. Nevertheless, further prolongation of the residence time did not give any benefits to the yield of the desired product $\mathbf{A}$ (Graph 2). 
Graph 2. Residence time optimization for the reaction of $p$-anisylmagnesium bromide (2a) with ethyl trifluoroacetate (7).

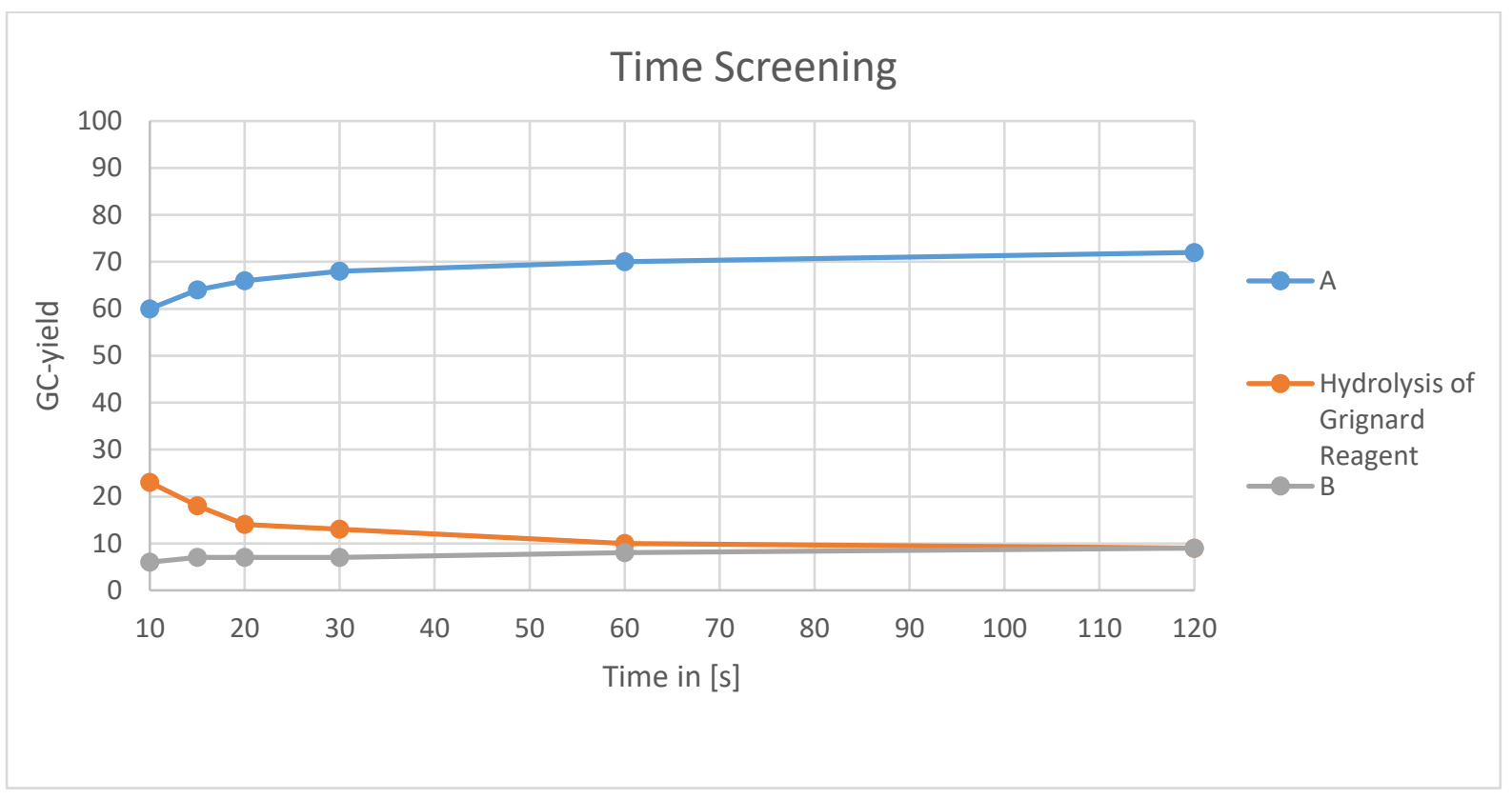

Graph 3. Ester equivalents optimization for the reaction of $p$-anisylmagnesium bromide (2a) with ethyl trifluoroacetate (7).

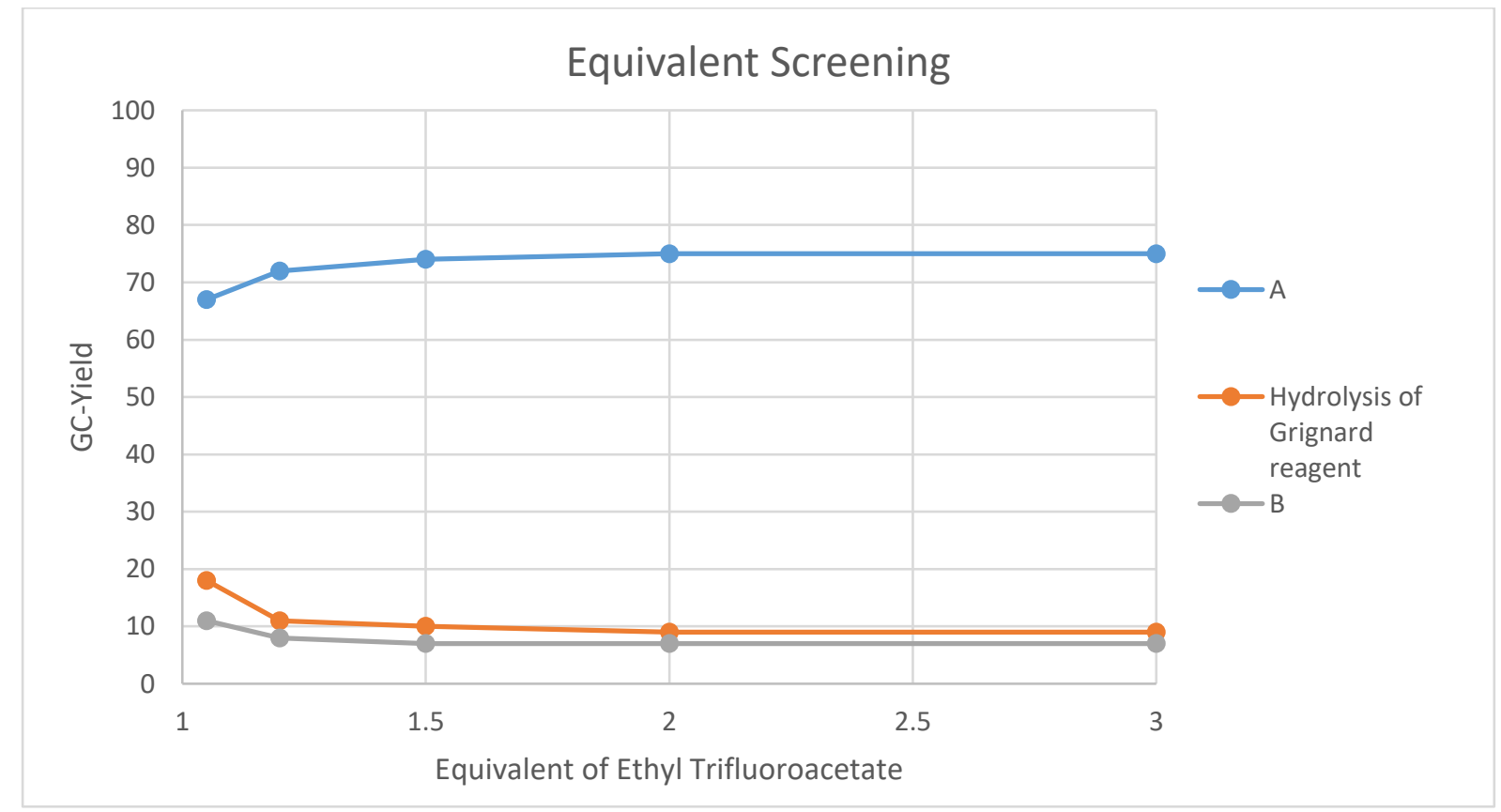

Superior mixing in flow enabled the use of nearly stoichiometric amounts of both reactants. Even increase from 1.2 equiv to 1.5 equiv gave really small improvements ( $72 \%$ to $74 \%$ calibrated GC yield of A, Graph 3). 
Table 1: Flowrate optimization optimization for the reaction of $p$-anisylmagnesium bromide (2a) with ethyl trifluoroacetate (7).

\begin{tabular}{cccccc} 
entry & $\begin{array}{c}\text { Flowrate } \\
{[\mathrm{mL} / \mathrm{min}]}\end{array}$ & $\mathrm{T}\left[{ }^{\circ} \mathrm{C}\right]$ & $\mathrm{t}[\mathrm{min}]$ & $\begin{array}{c}\text { yield } \mathbf{A} \\
{[\mathrm{GC}-\%]}\end{array}$ & $\begin{array}{c}\text { yield } \mathbf{B} \\
{[\mathrm{GC}-\%]}\end{array}$ \\
\hline 1 & 2 & -5 & 2 & 56 & 13 \\
2 & 5 & -5 & 2 & 65 & 10 \\
3 & 10 & -5 & 2 & 72 & 8 \\
4 & 20 & -5 & 2 & 72 & 8
\end{tabular}

Increasing flow rate at constant residence time $(2 \mathrm{~min})$ showed positive impact on ketone/tertiary alcohol ratio. By comparing entry 1 and entry 3 it can be seen that selectivity increased is more than two-fold. However, further increase of flow rate caused pressure shutdown problems and did not bring any benefits in selectivity or conversion. 
${ }^{1} \mathrm{H}-\mathrm{NMR},{ }^{19} \mathrm{~F}-\mathrm{NMR}$ and ${ }^{13} \mathrm{C}$-NMR Spectra of Products

2,2,2-Trifluoro-1-(4-methoxyphenyl)ethan-1-one (6a)<smiles>COc1ccc(C(=O)C(F)(F)F)cc1</smiles>
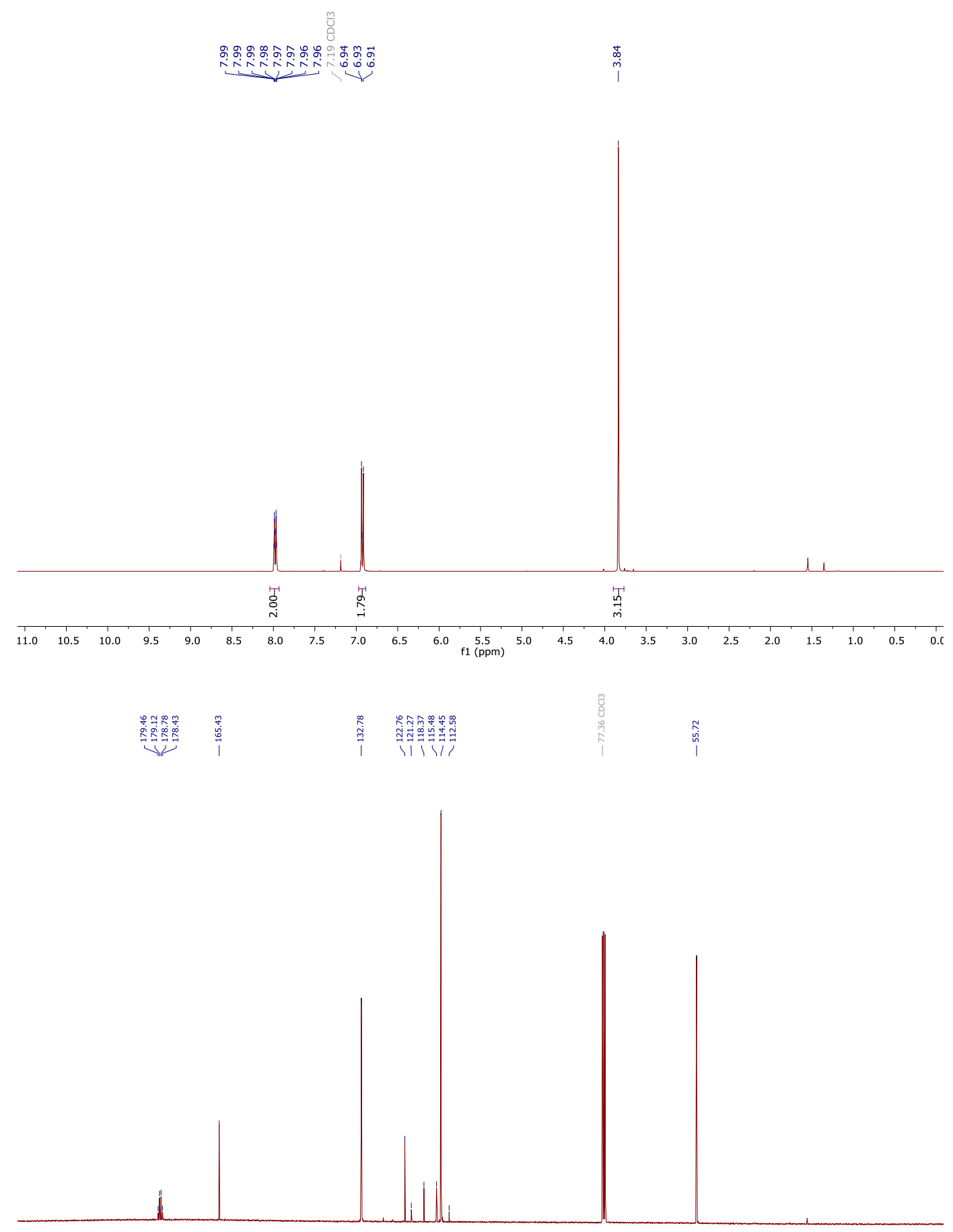

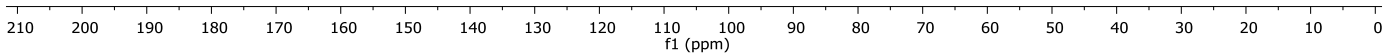


2,2,2-Trifluoro-1-(3-methoxyphenyl)ethan-1-one (6b)<smiles>COc1cccc(C(=O)C(F)(F)F)c1</smiles>

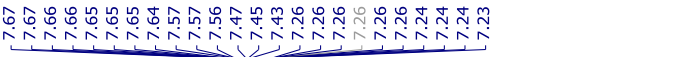

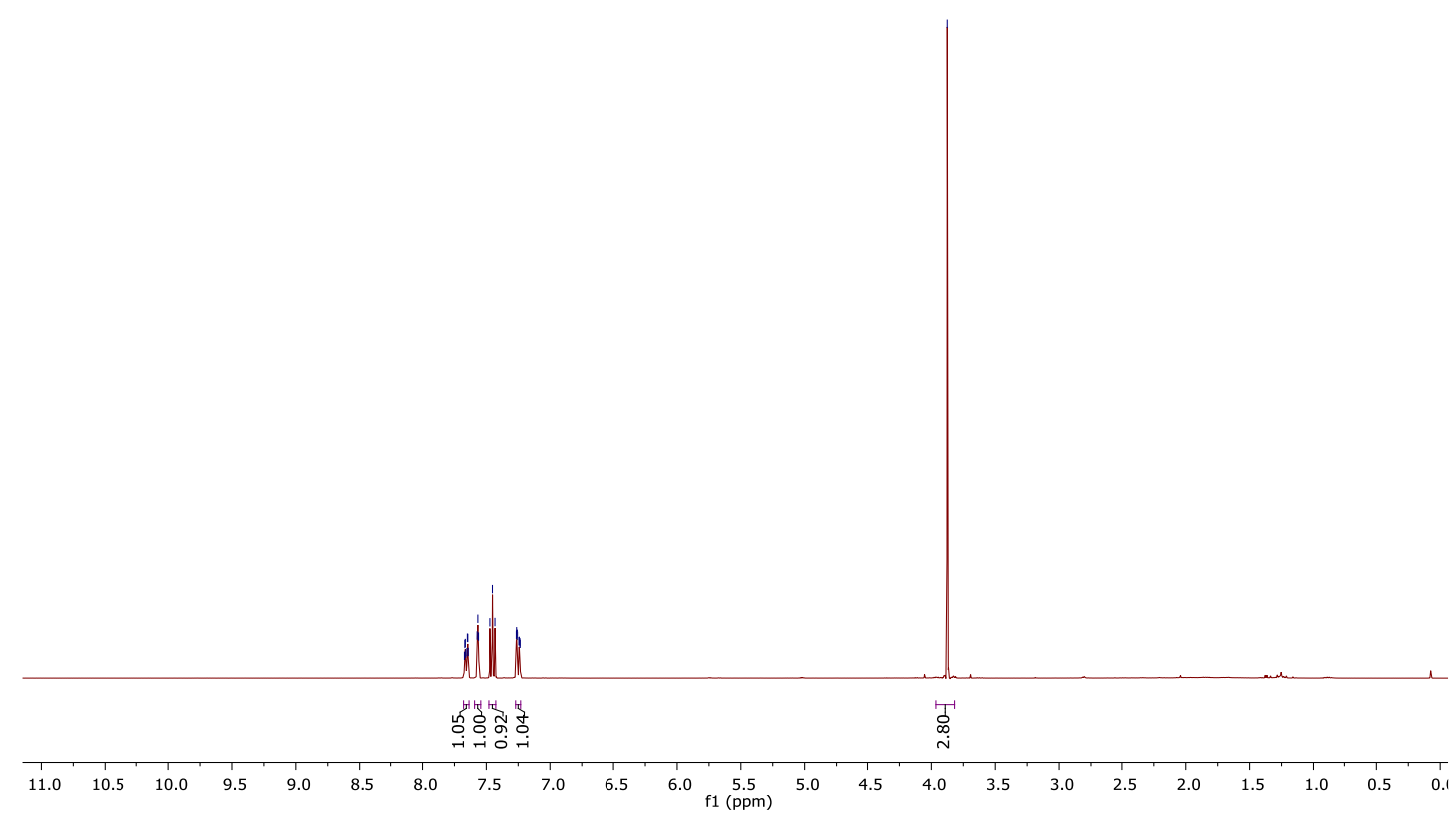

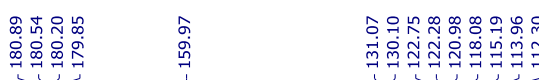

负

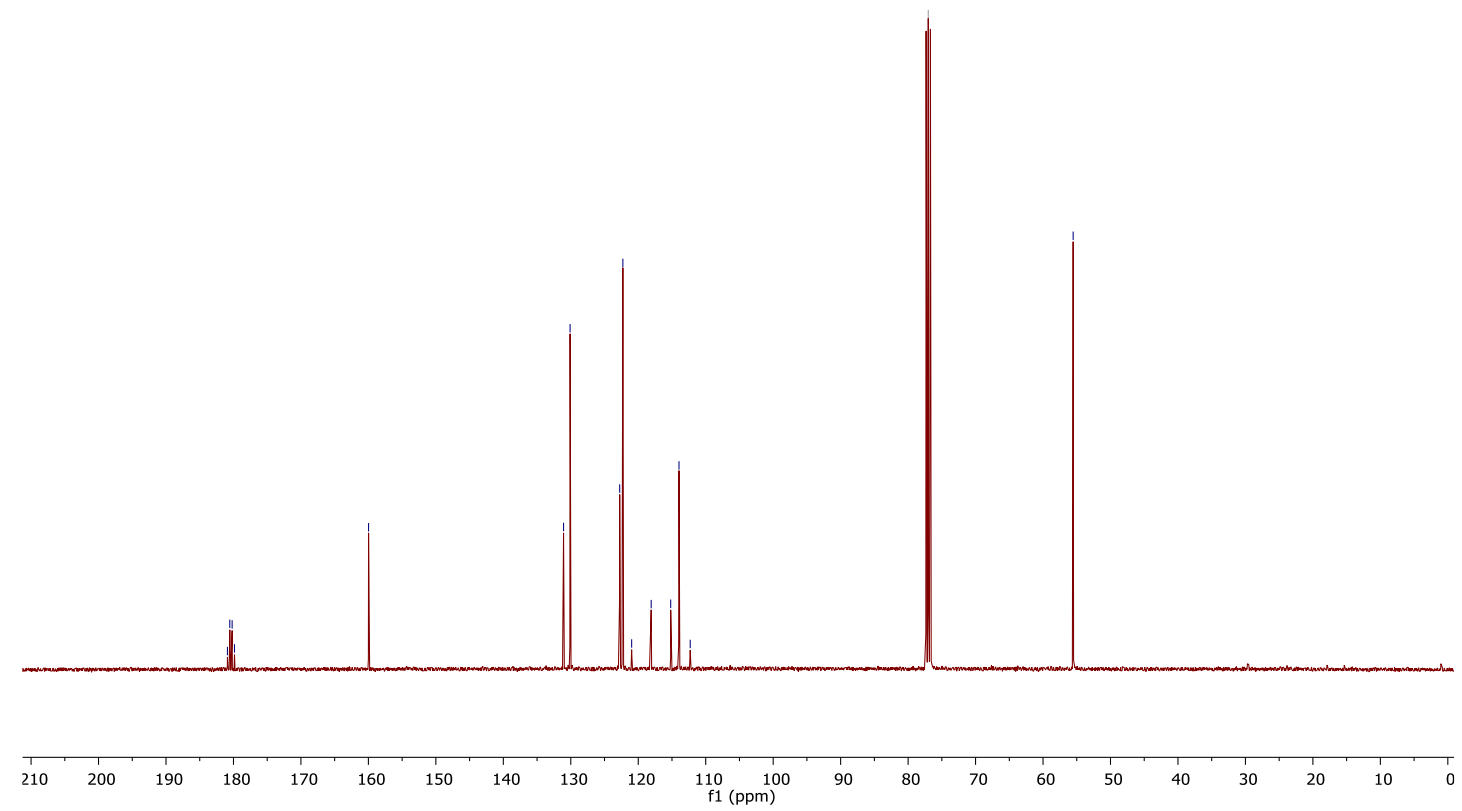


2,2,2-Trifluoro-1-(2-methoxyphenyl)ethan-1-one (6c)

$\mathrm{CF}_{3}$
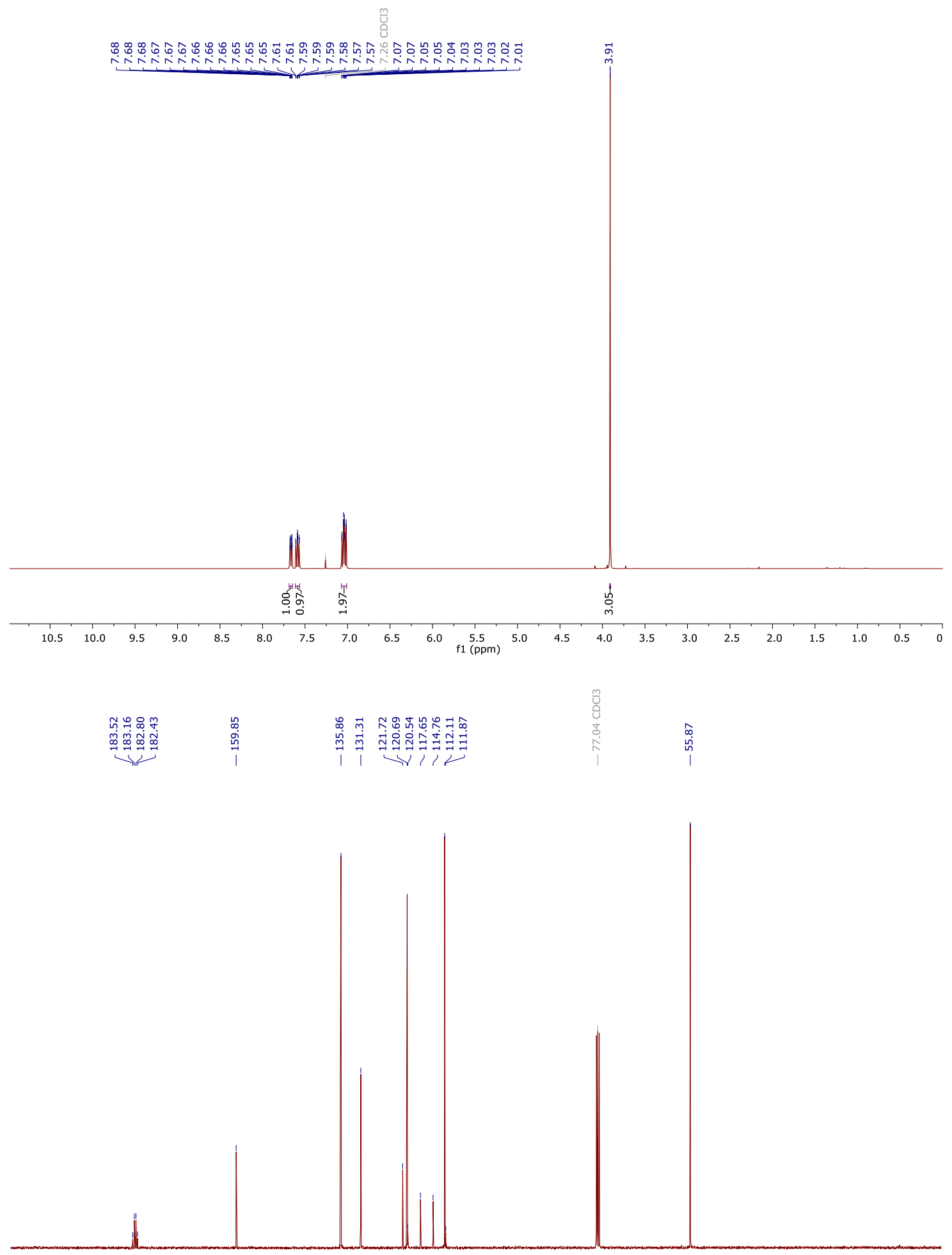

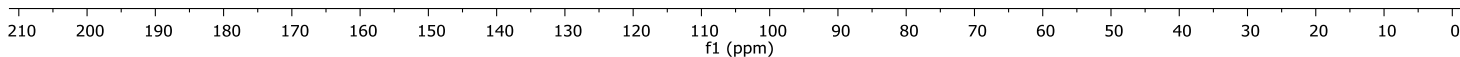




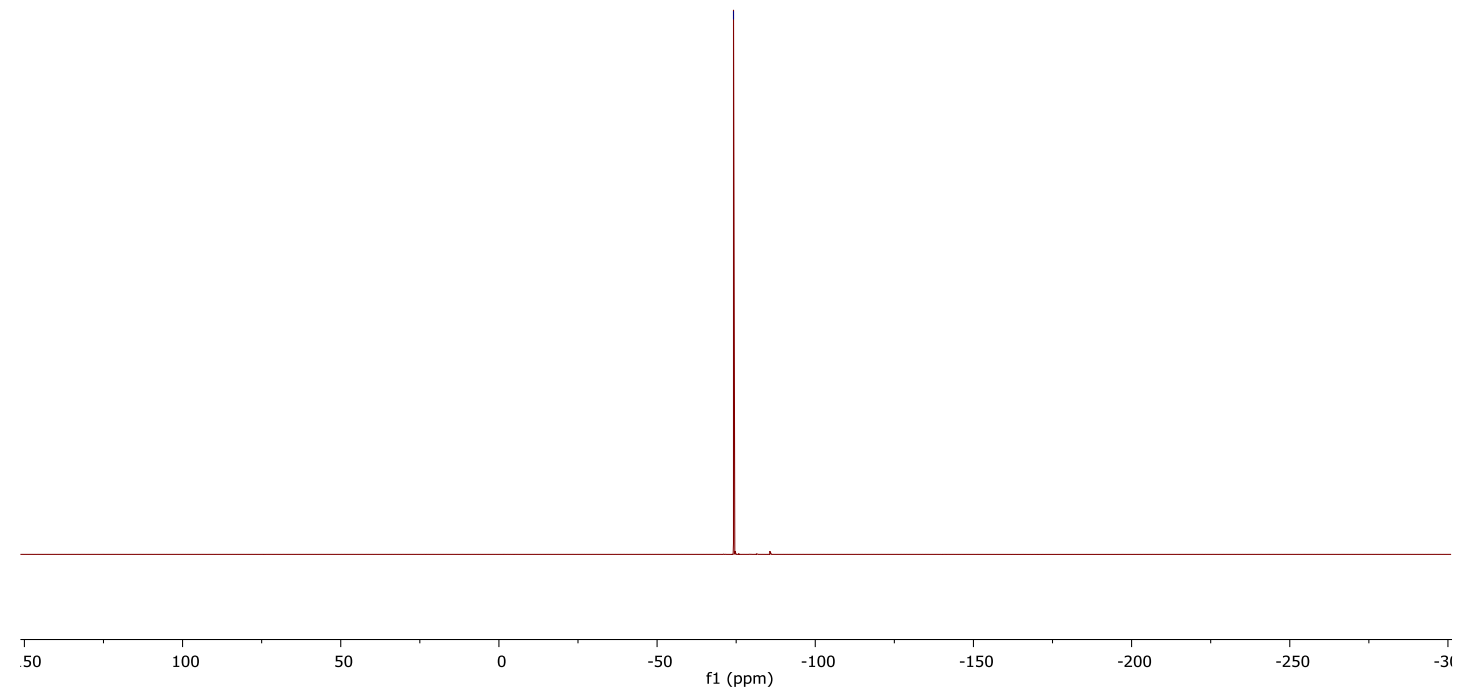


1-(Benzo[d][1,3]dioxol-5-yl)-2,2,2-trifluoroethan-1-one (6d)<smiles>O=C(c1ccc2c(c1)OCO2)C(F)(F)F</smiles>

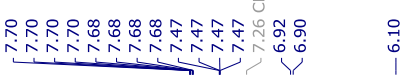
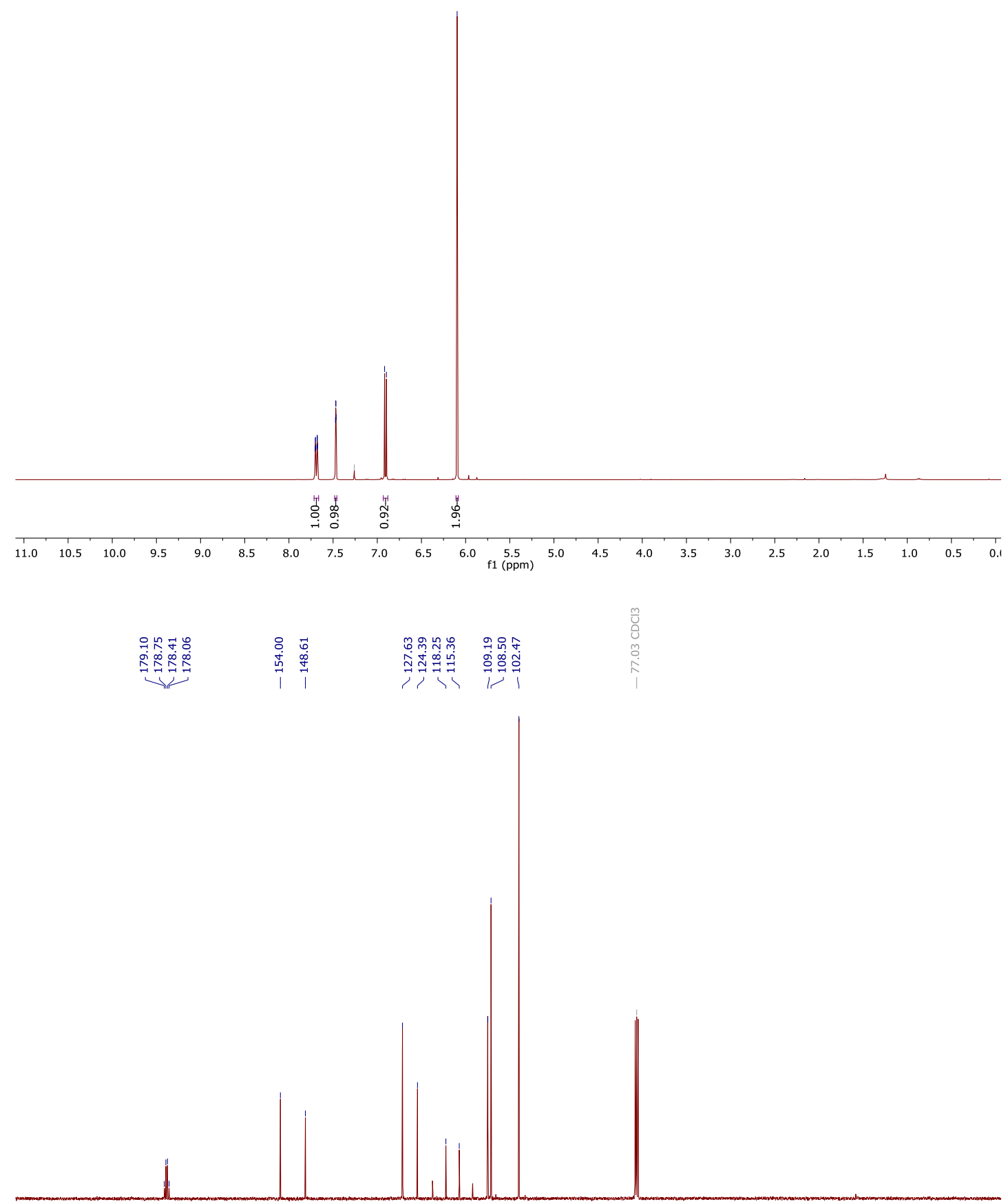

$\begin{array}{lllllllllll}210 & 200 & 190 & 180 & 170 & 160 & 150 & 140 & 130 & 120 & 110 \\ \mathrm{f} 1(\mathrm{ppm}) & 100\end{array}$ 
2,2,2-Trifluoro-1-(6-methoxynaphthalen-2-yl)ethan-1-one (6e)<smiles>COc1ccc2cc(C(=O)C(F)(F)C(F)(F)F)ccc2c1</smiles>
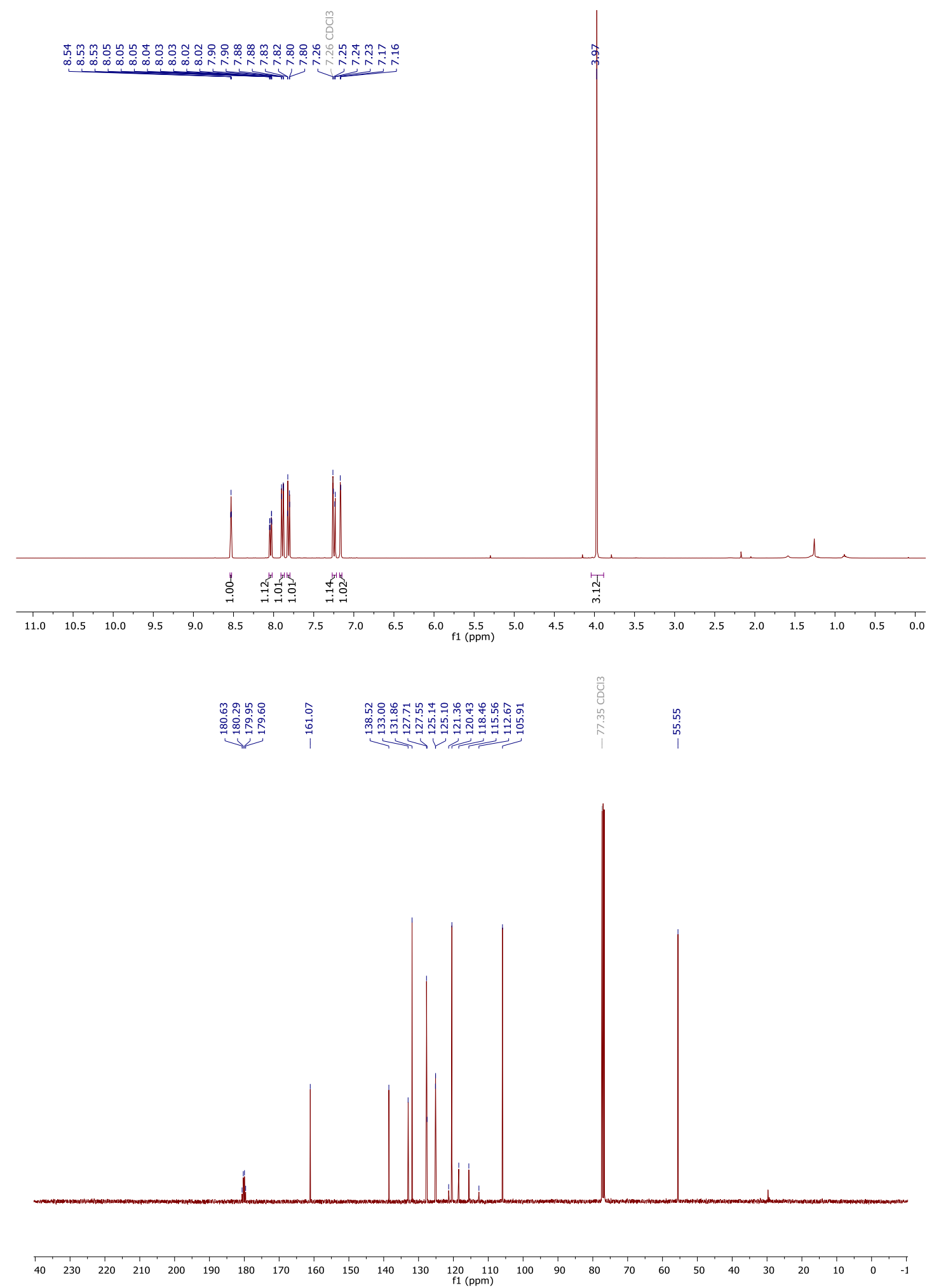


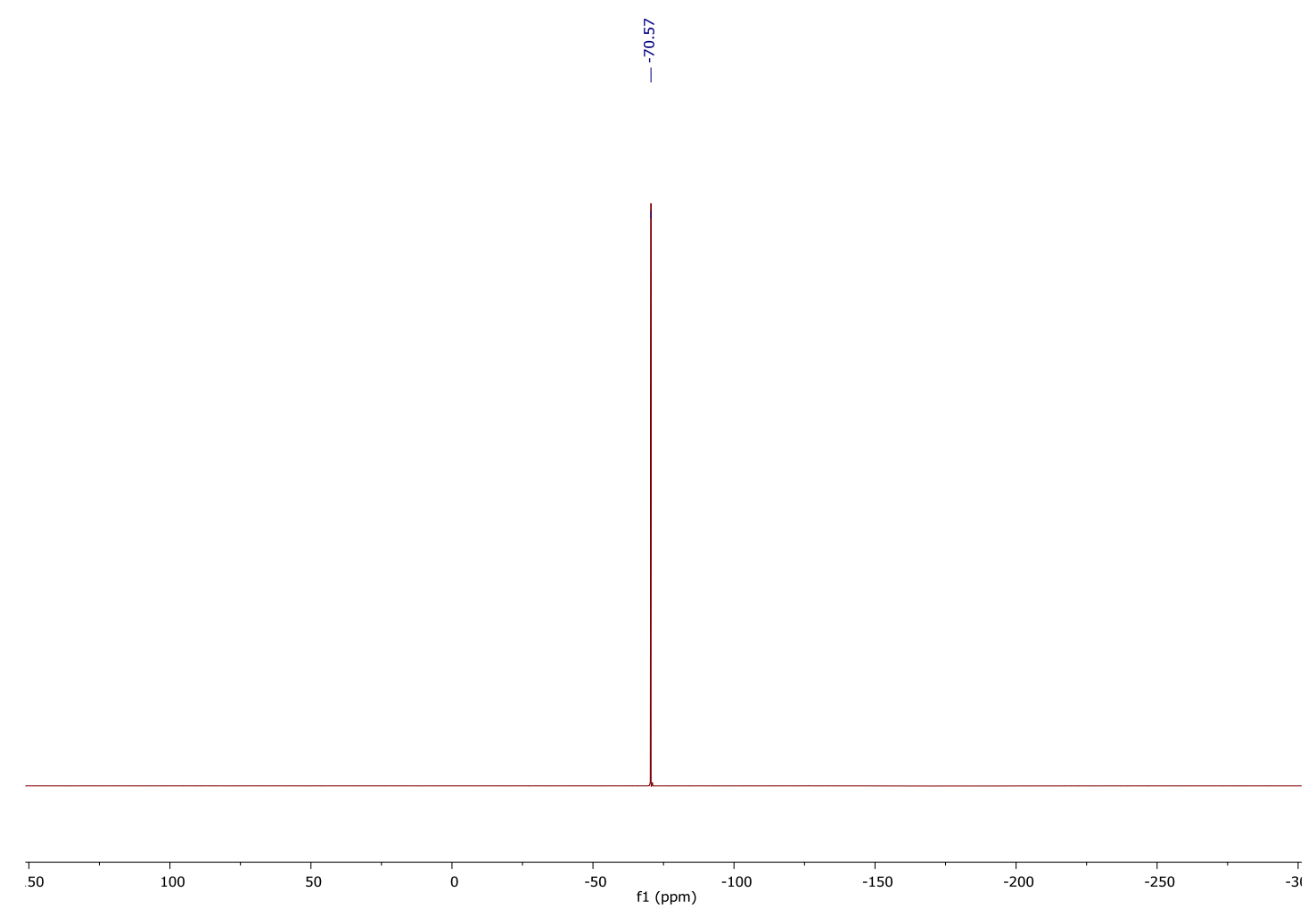


1-(3,5-Di-tert-butylphenyl)-2,2,2-trifluoroethan-1-one (6f)<smiles>CC(C)(C)c1cc(C(=O)C(F)(F)F)cc(C(C)(C)C)c1</smiles>
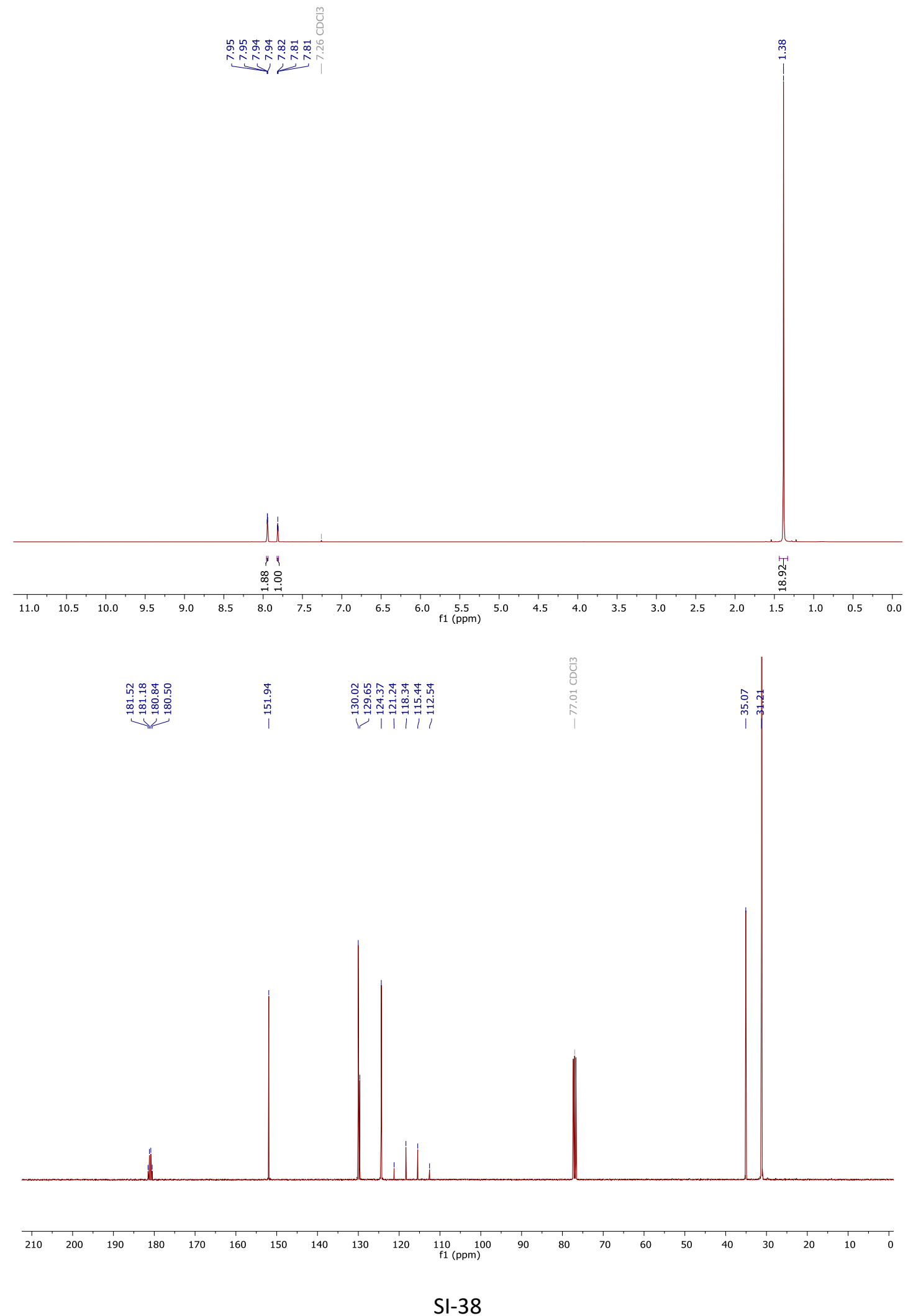


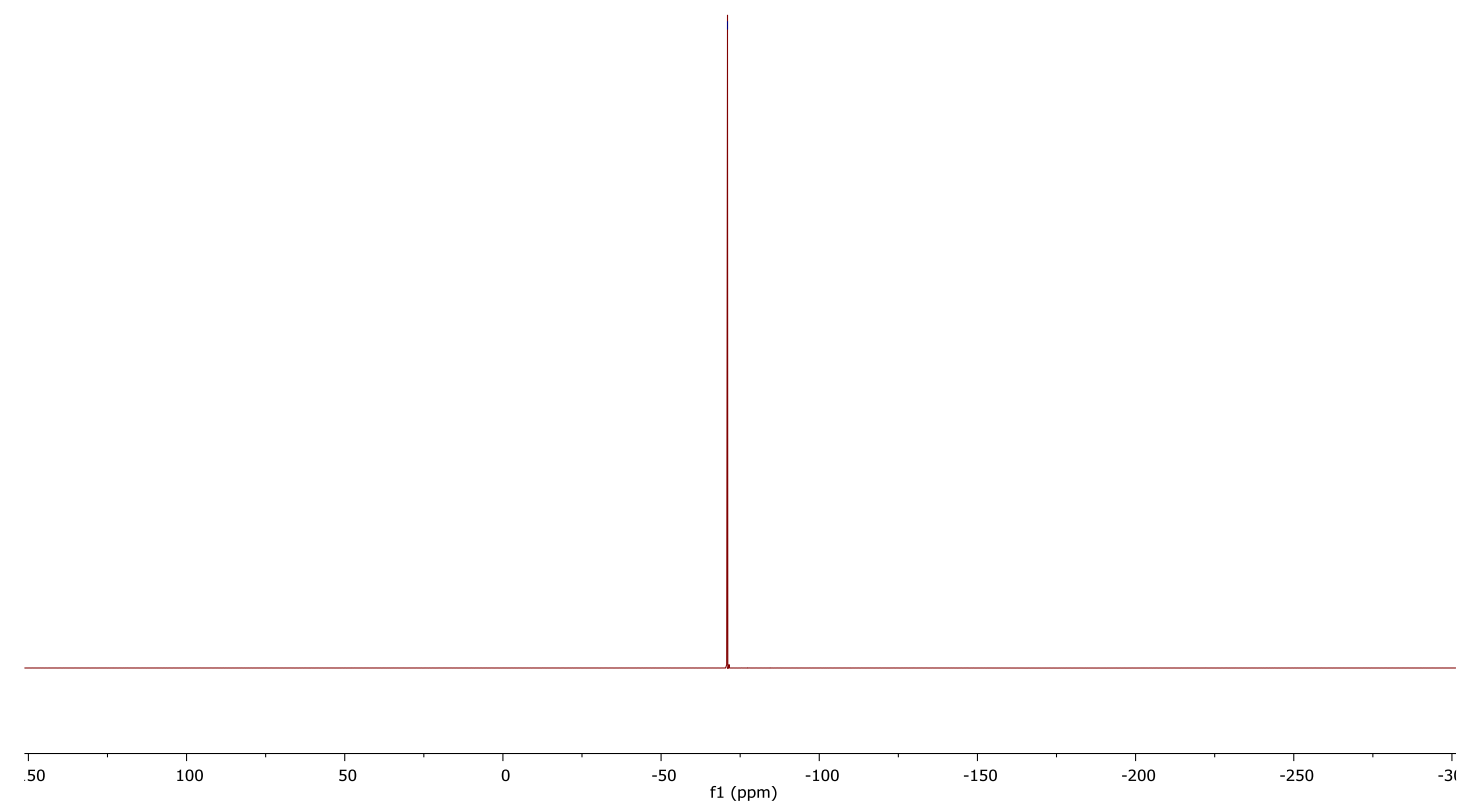


1-(Benzo[b]thiophen-2-yl)-2,2,2-trifluoroethan-1-one (6g)<smiles>O=C(c1cc2ccccc2s1)C(F)(F)F</smiles>

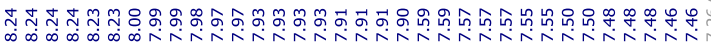

年
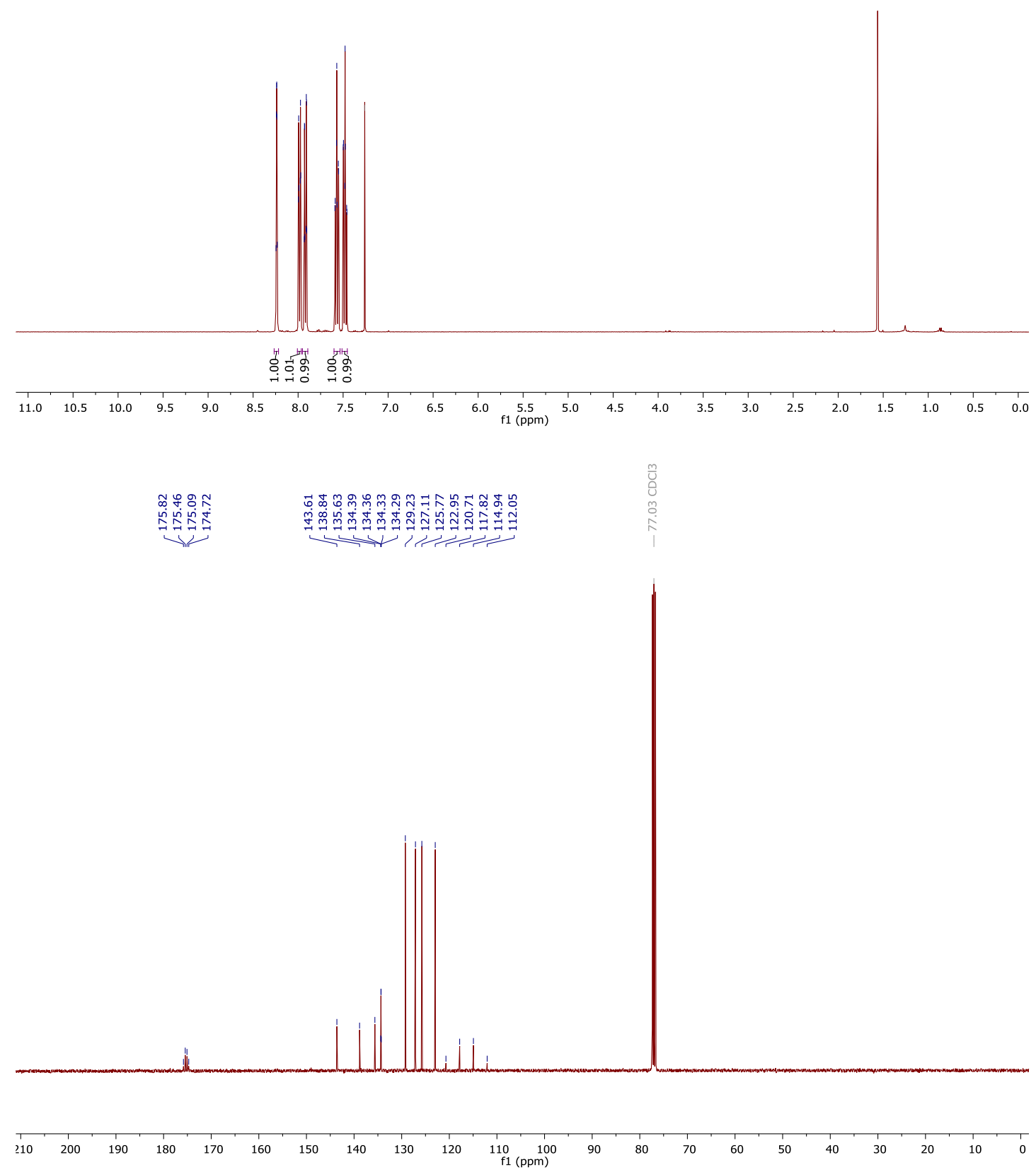


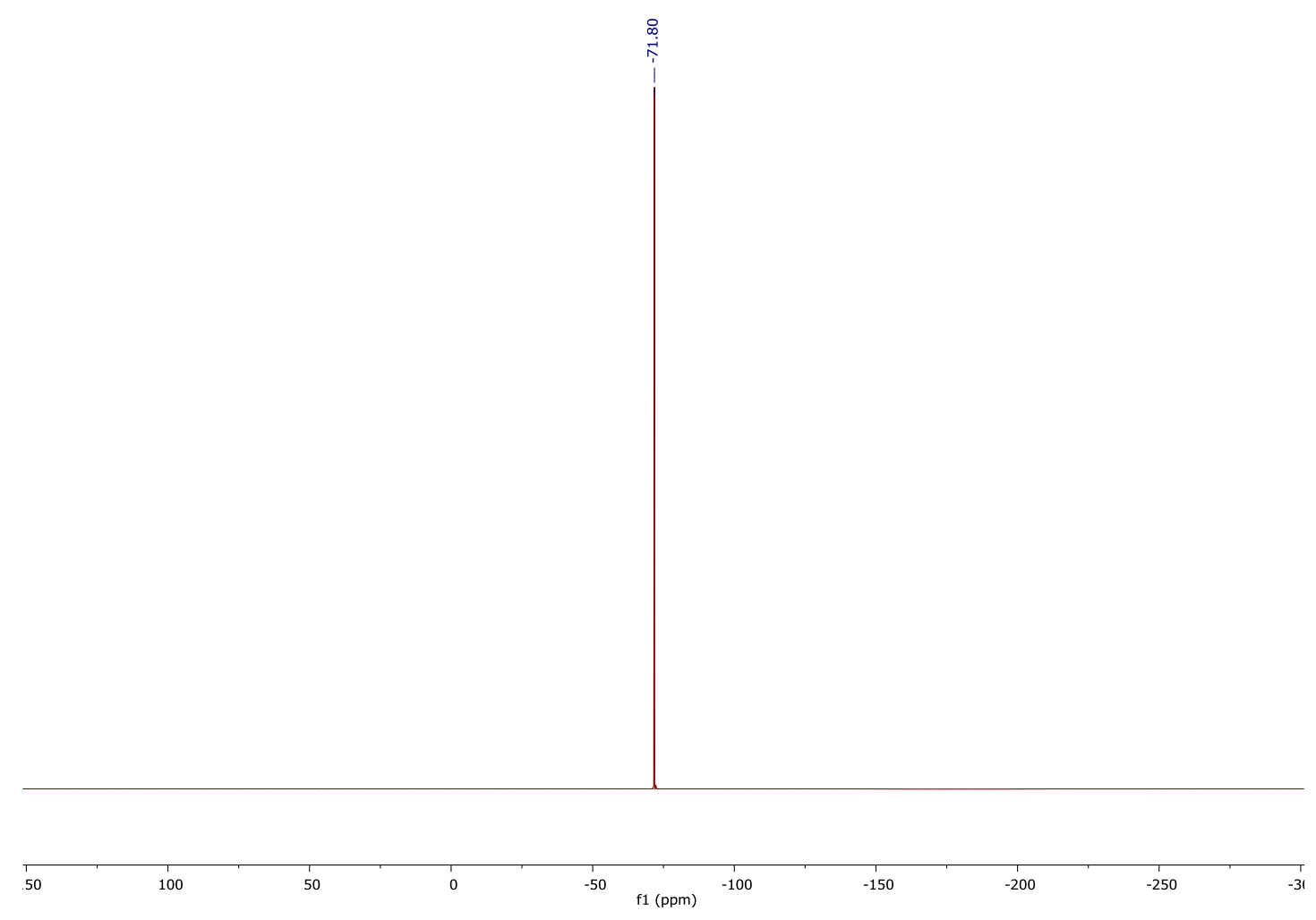


2,2,2-Trifluoro-1-(1-methyl-1H-indol-5-yl)ethan-1-one (6h)<smiles>Cn1ccc2cc(C(=O)C(F)(F)F)ccc21</smiles>

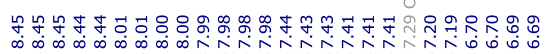

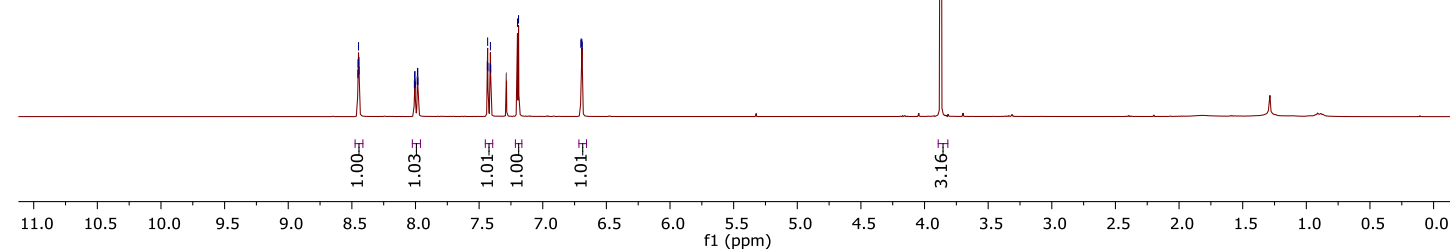




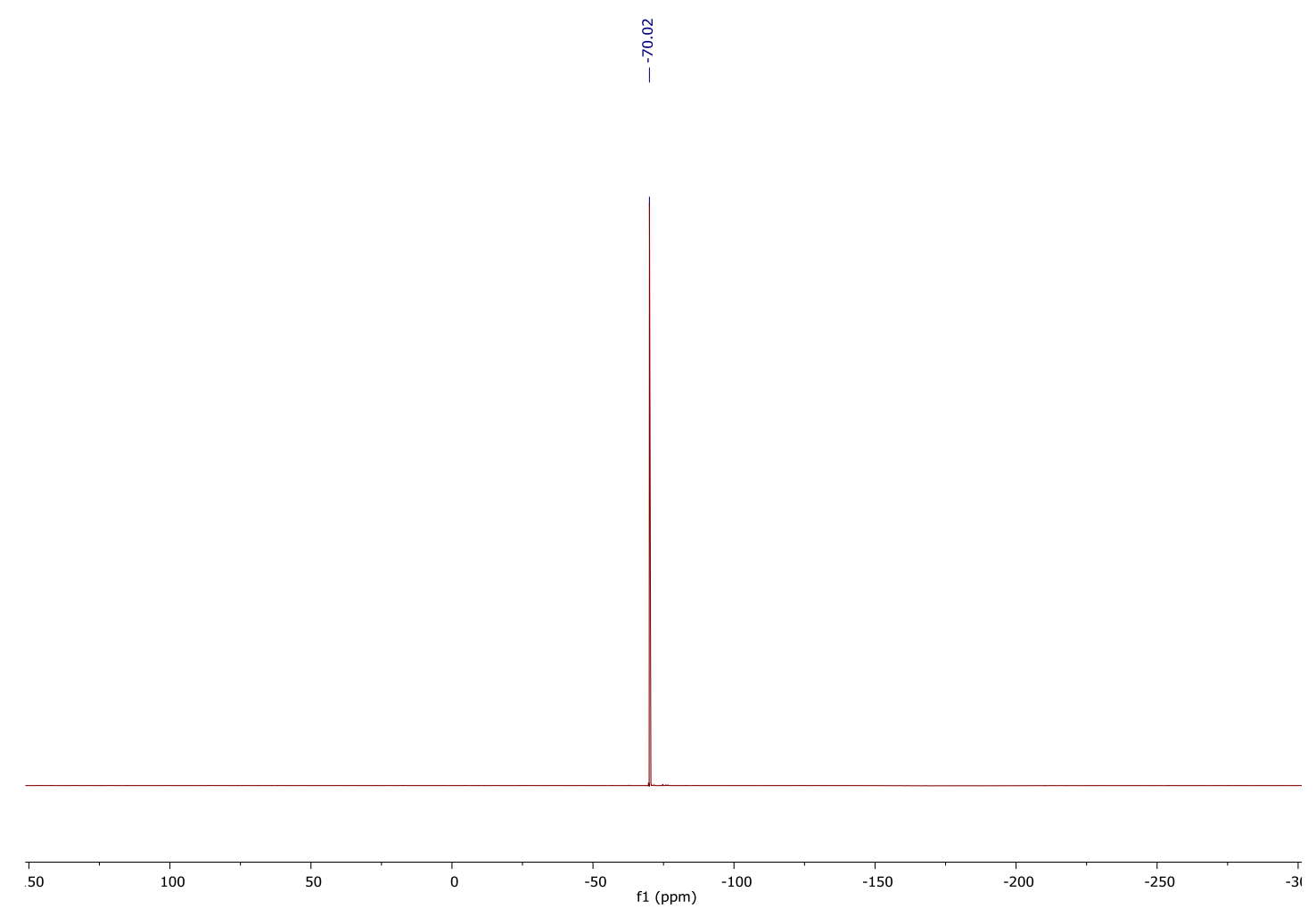


Ethyl 4-(2,2,2-trifluoroacetyl)benzoate (6i)<smiles>CCOC(=O)c1ccc(C(=O)C(F)(F)F)cc1</smiles>

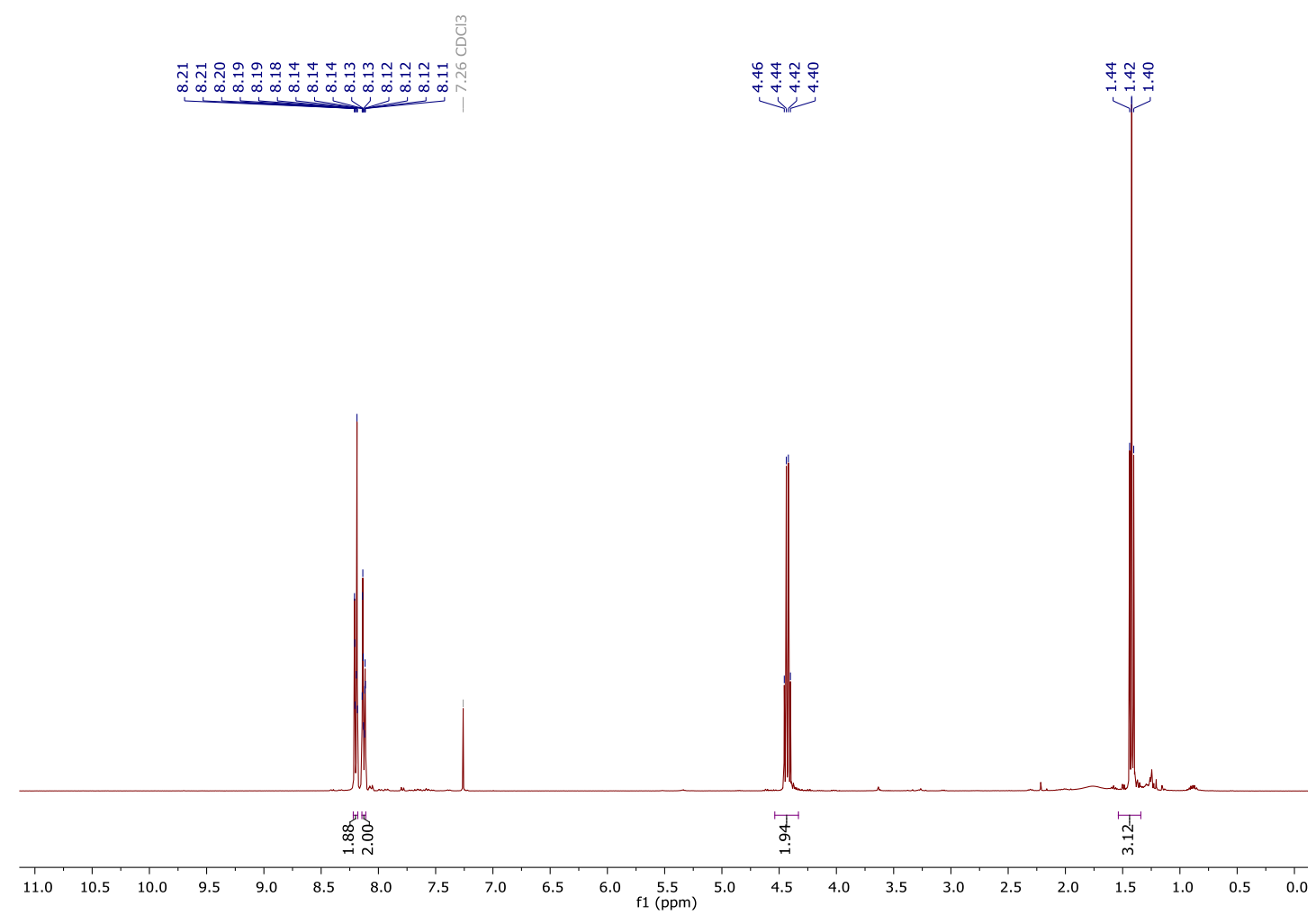

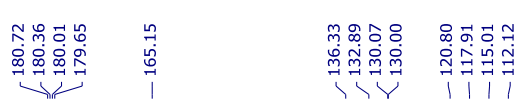

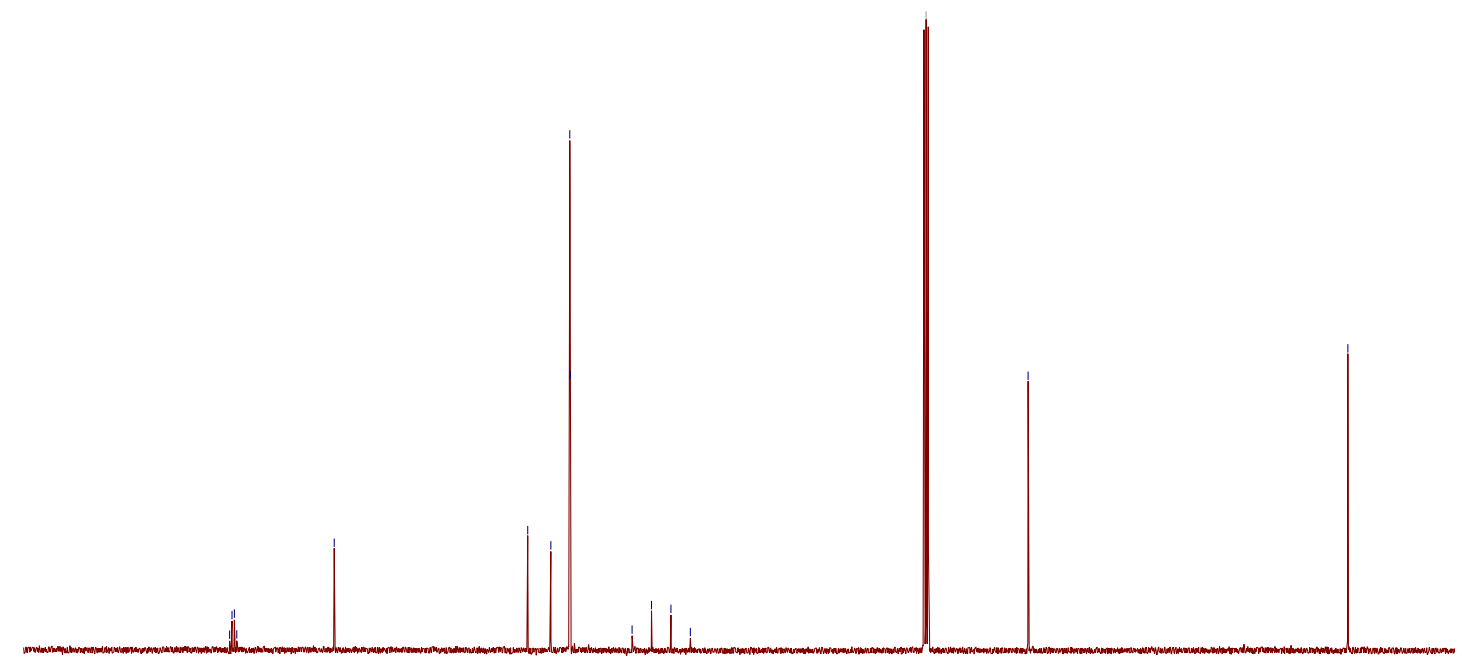

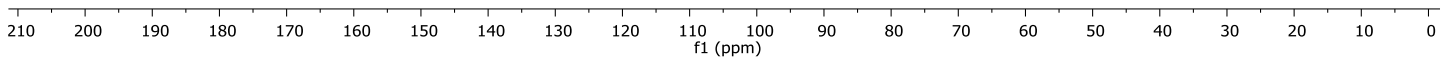




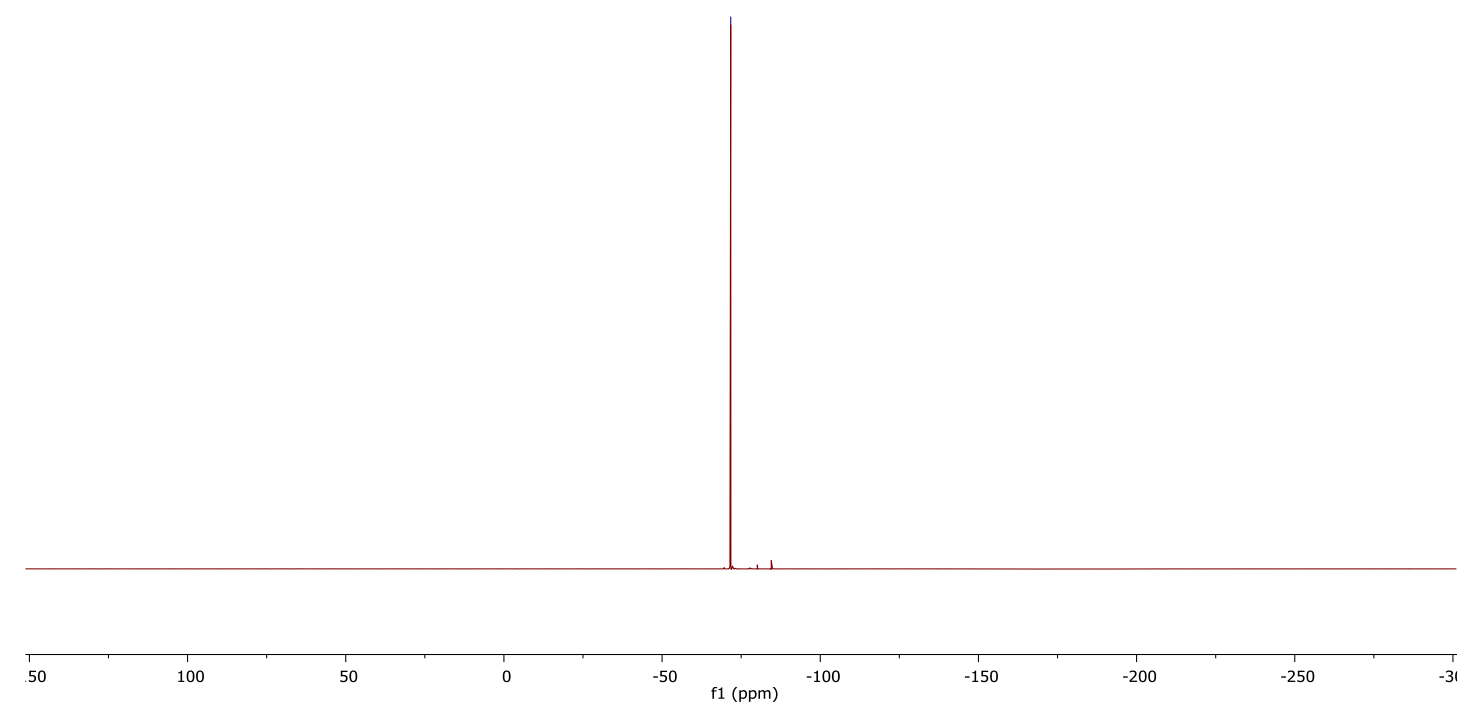


(3-Methoxyphenyl)(pyridin-2-yl)methanone (10a)<smiles>COc1cccc(C(=O)c2ccccn2)c1</smiles>

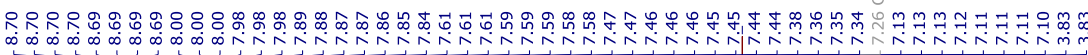

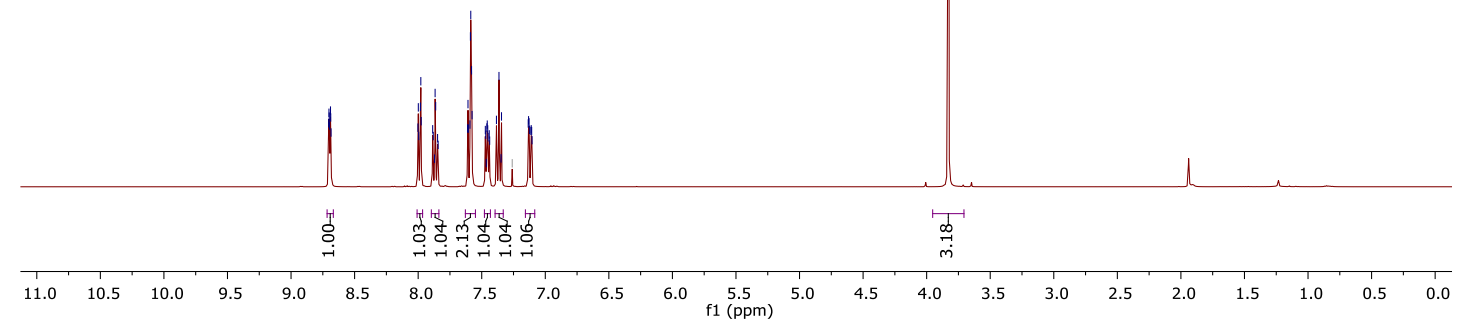

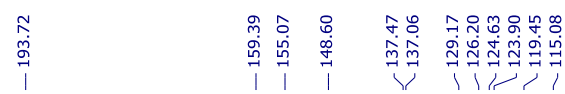

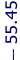

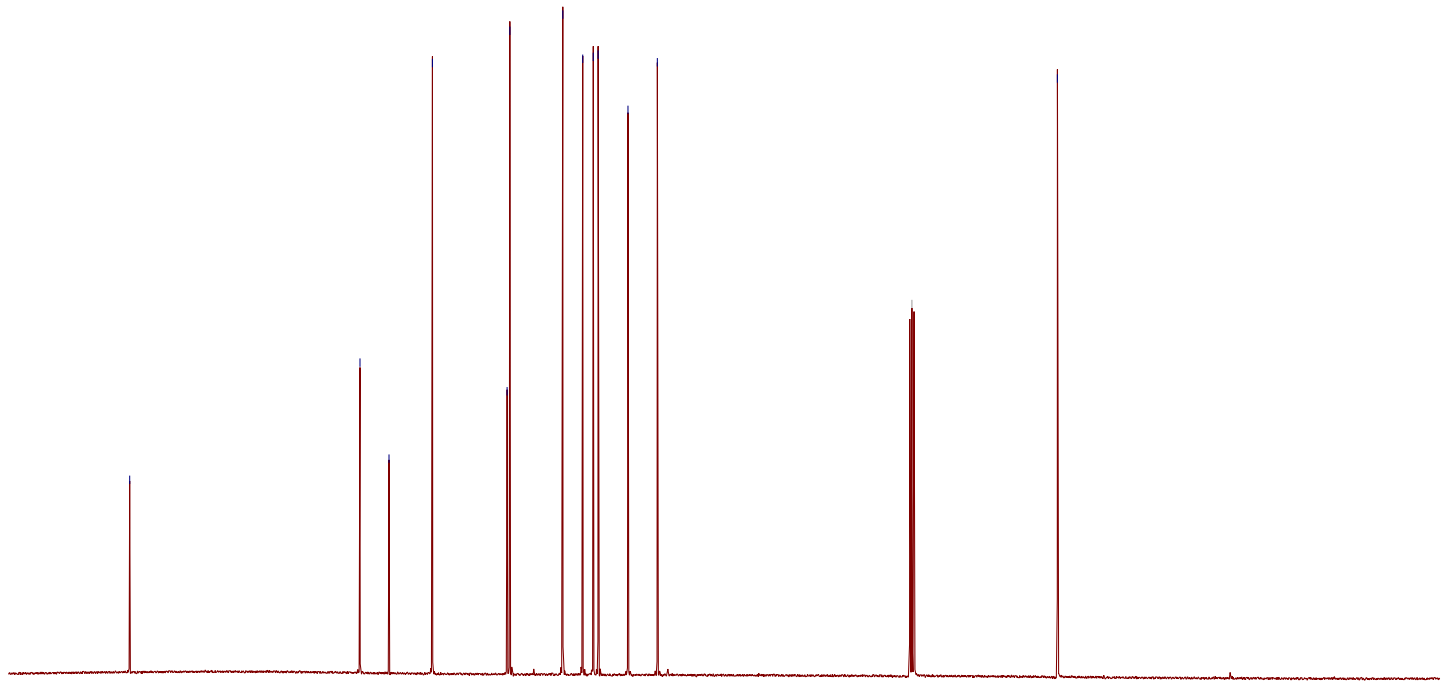

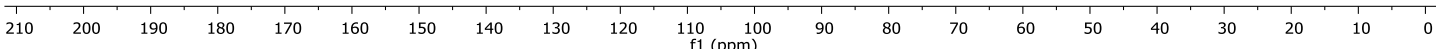




\section{4-Picolinoylbenzonitrile (10b)}<smiles>N#Cc1ccc(C(=O)c2ccccn2)cc1</smiles>

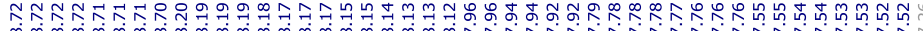

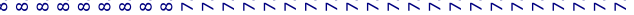

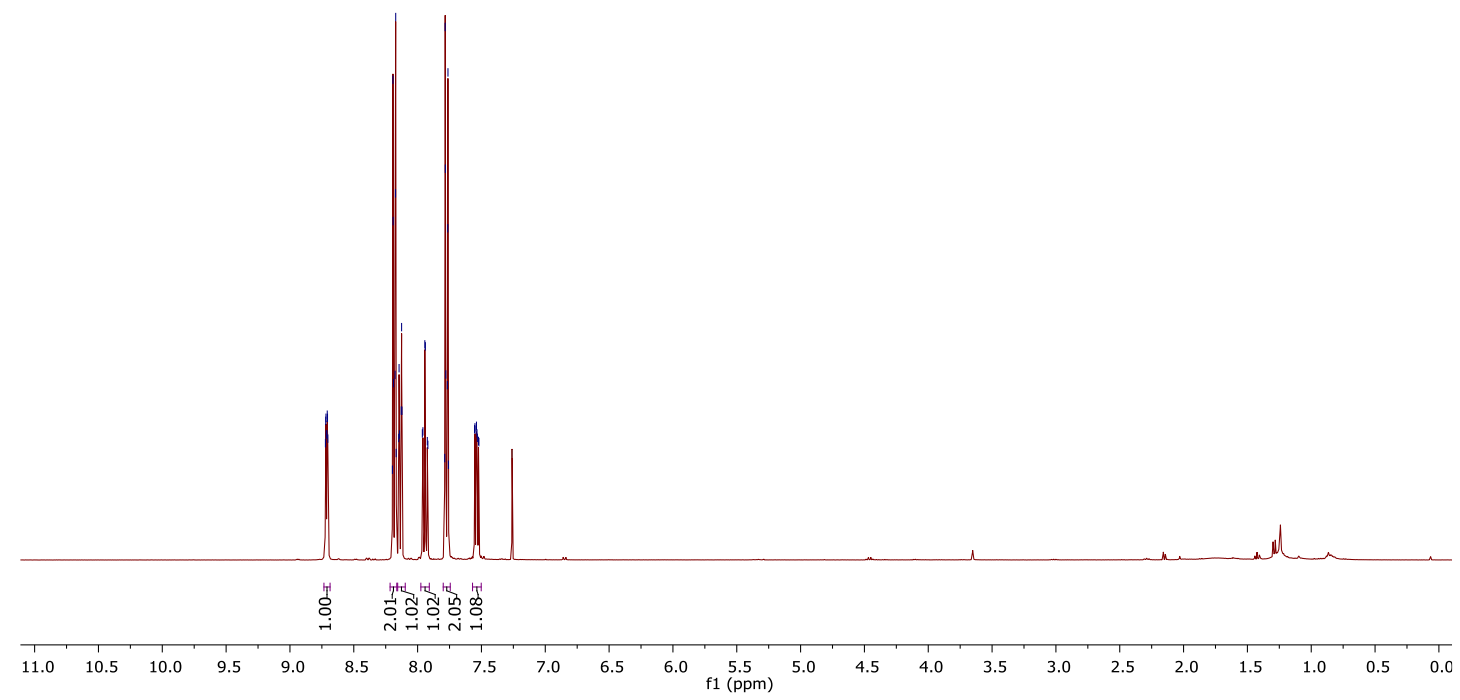

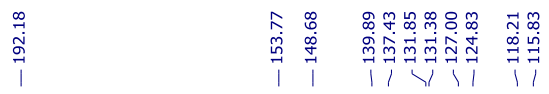

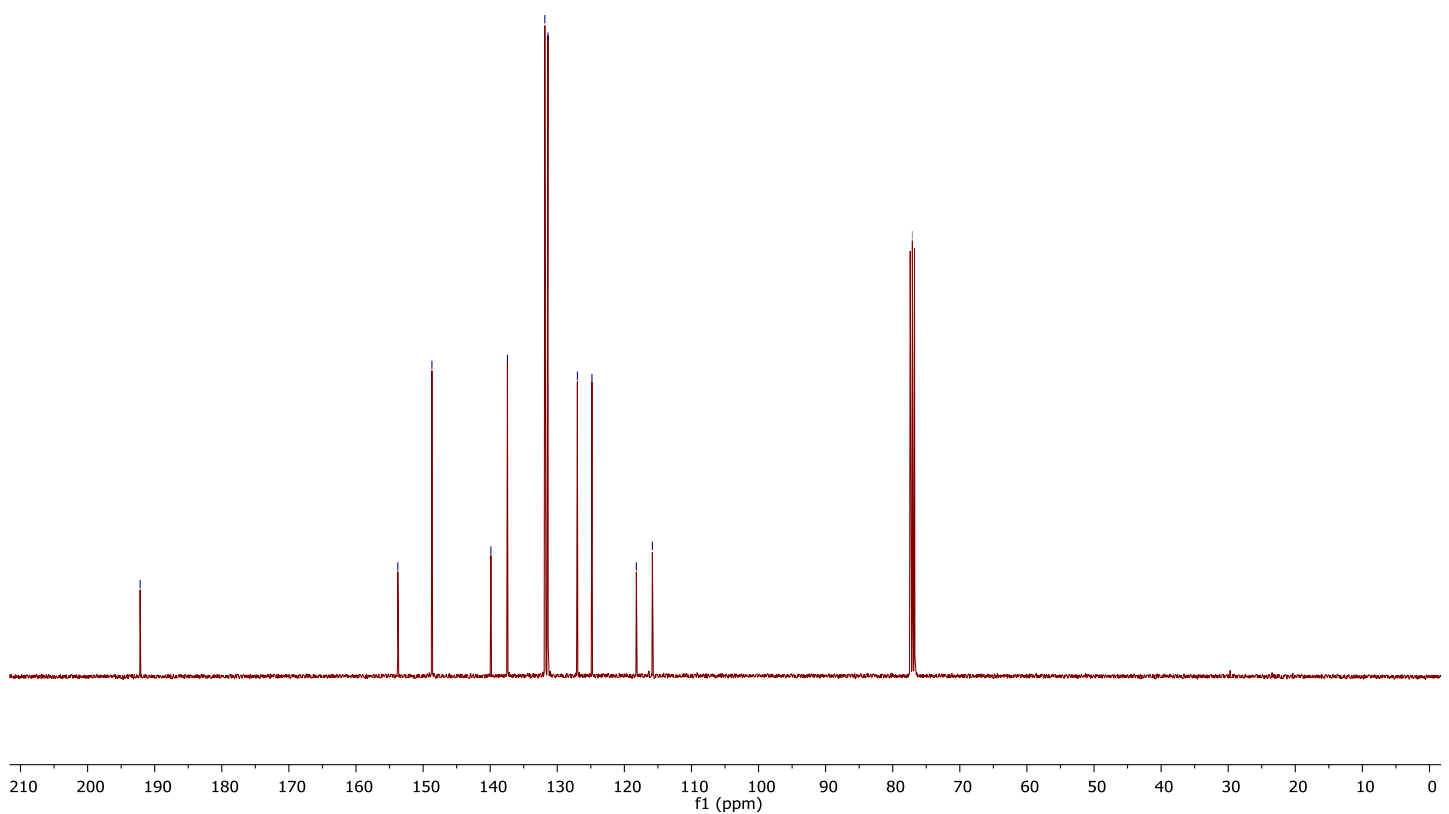


3-Phenyl-1-(pyridin-2-yl)propan-1-one (10c)<smiles>O=C(CCc1ccccc1)c1ccccn1</smiles>
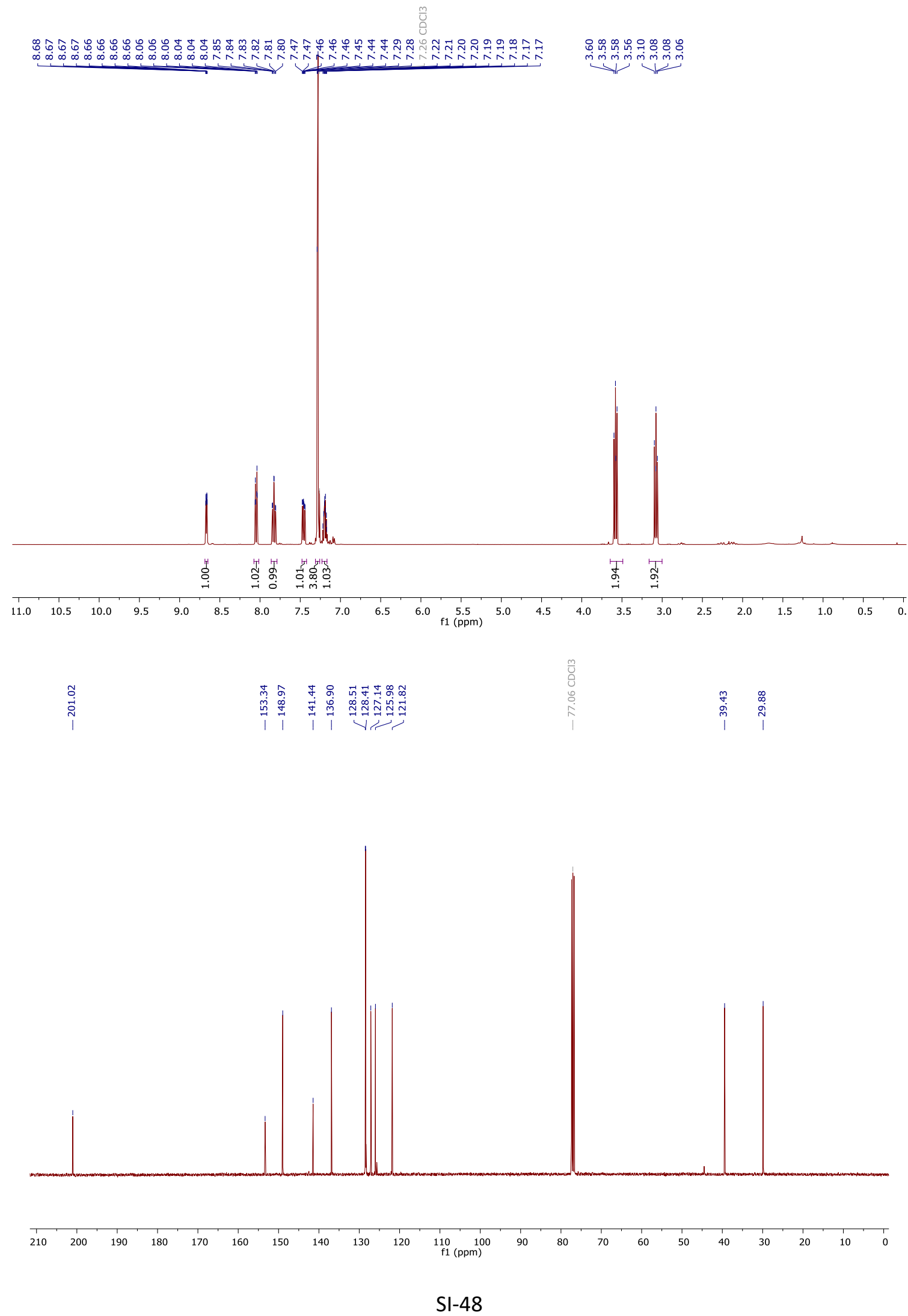
(2-(dimethylamino)phenyl)(pyrazin-2-yl)methanone (10d)<smiles>CN(C)c1ccccc1C(=O)c1cnccn1</smiles>

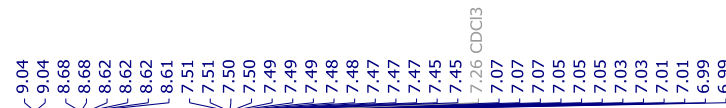

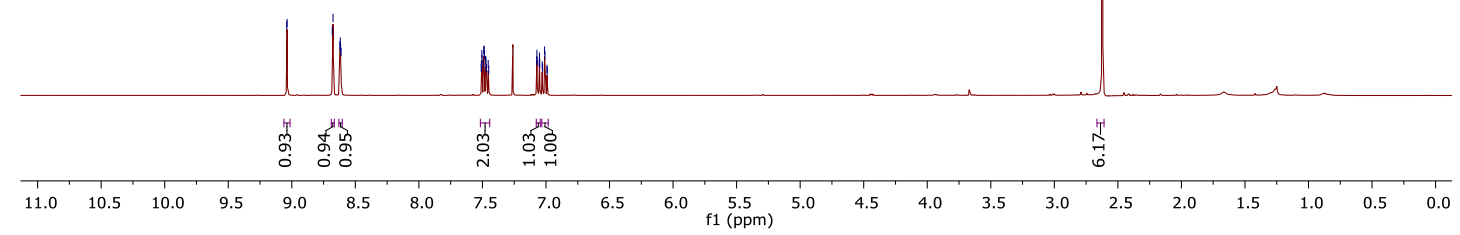

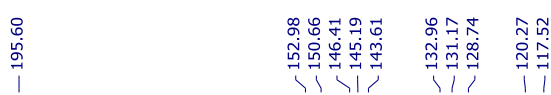

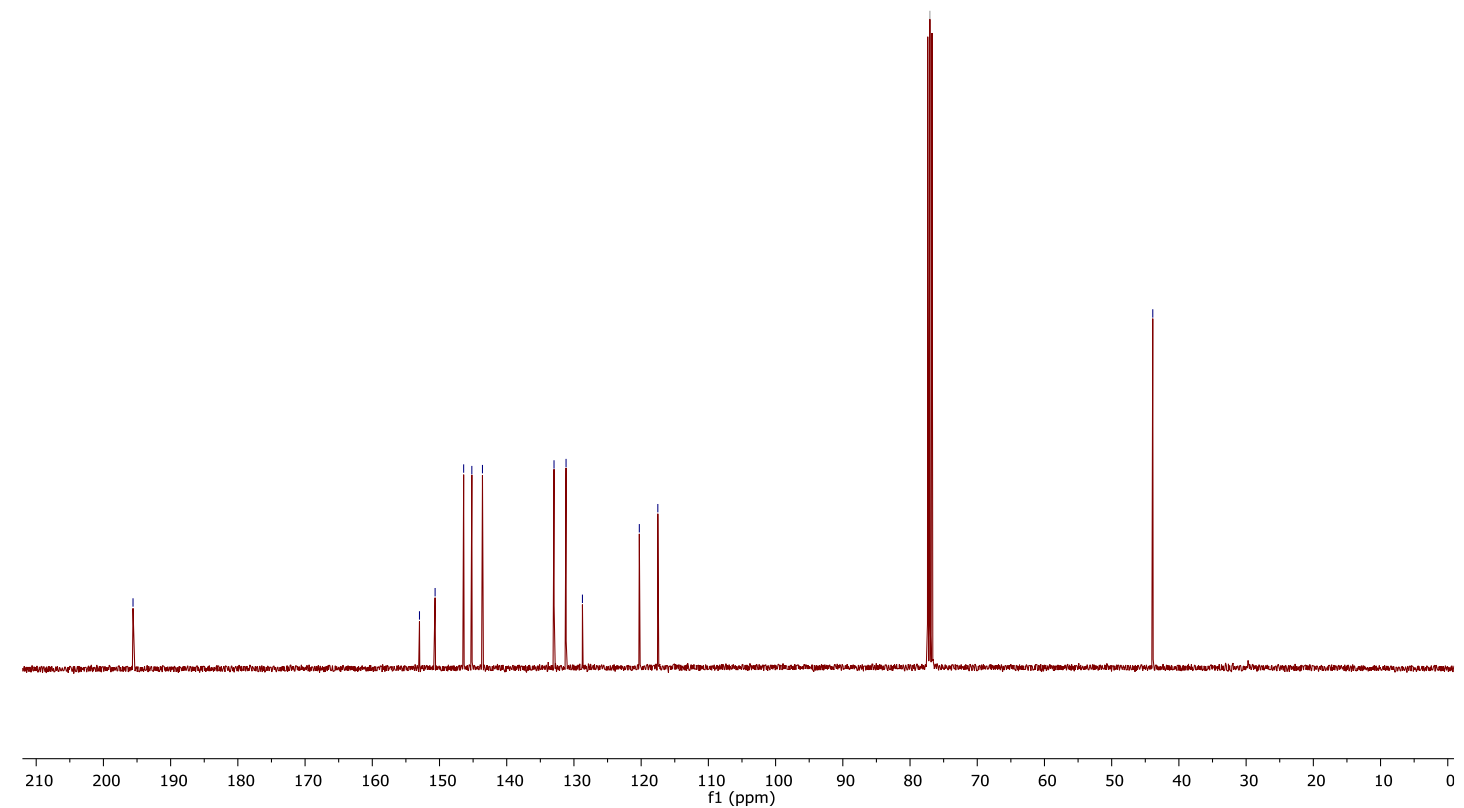


(3-Chlorophenyl)(pyrazin-2-yl)methanone (10e)<smiles>O=C(c1cccc(Cl)c1)c1cnccn1</smiles>

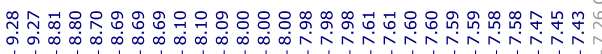

U.

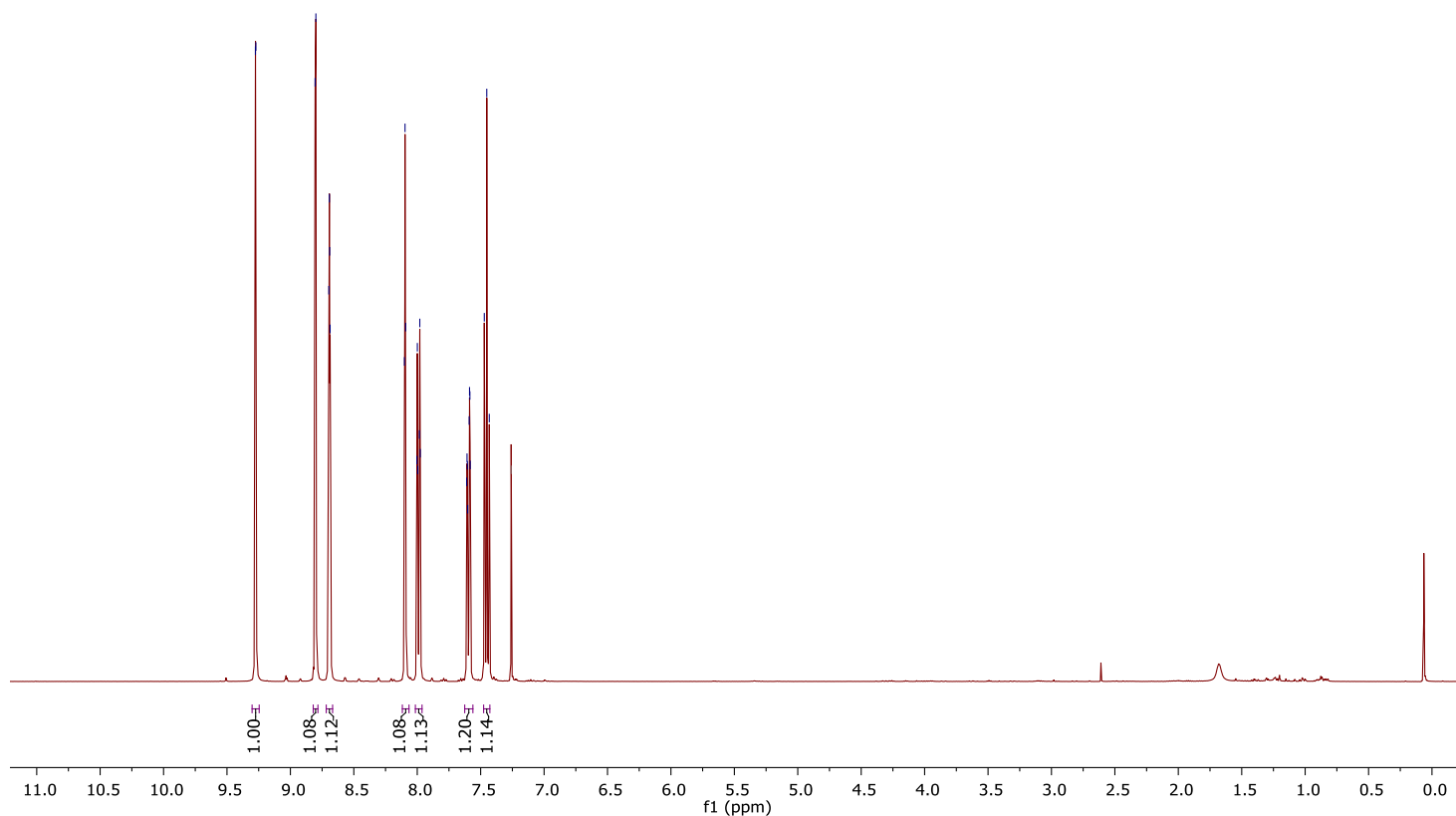

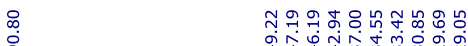

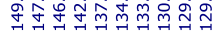

पो। सो।

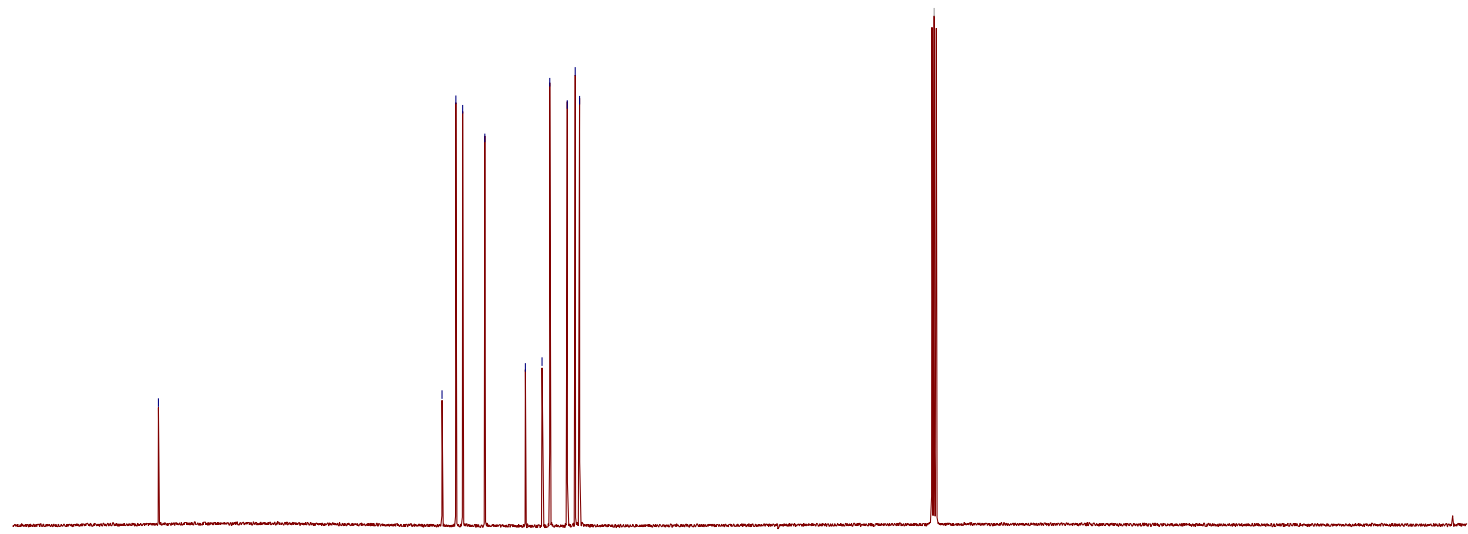

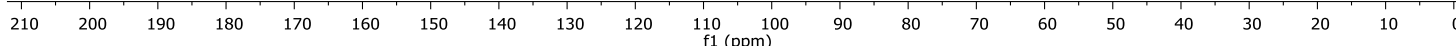


4-(pyrazine-2-carbonyl)benzonitrile (10f)<smiles>N#Cc1ccc(C(=O)c2cnccn2)cc1</smiles>

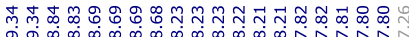

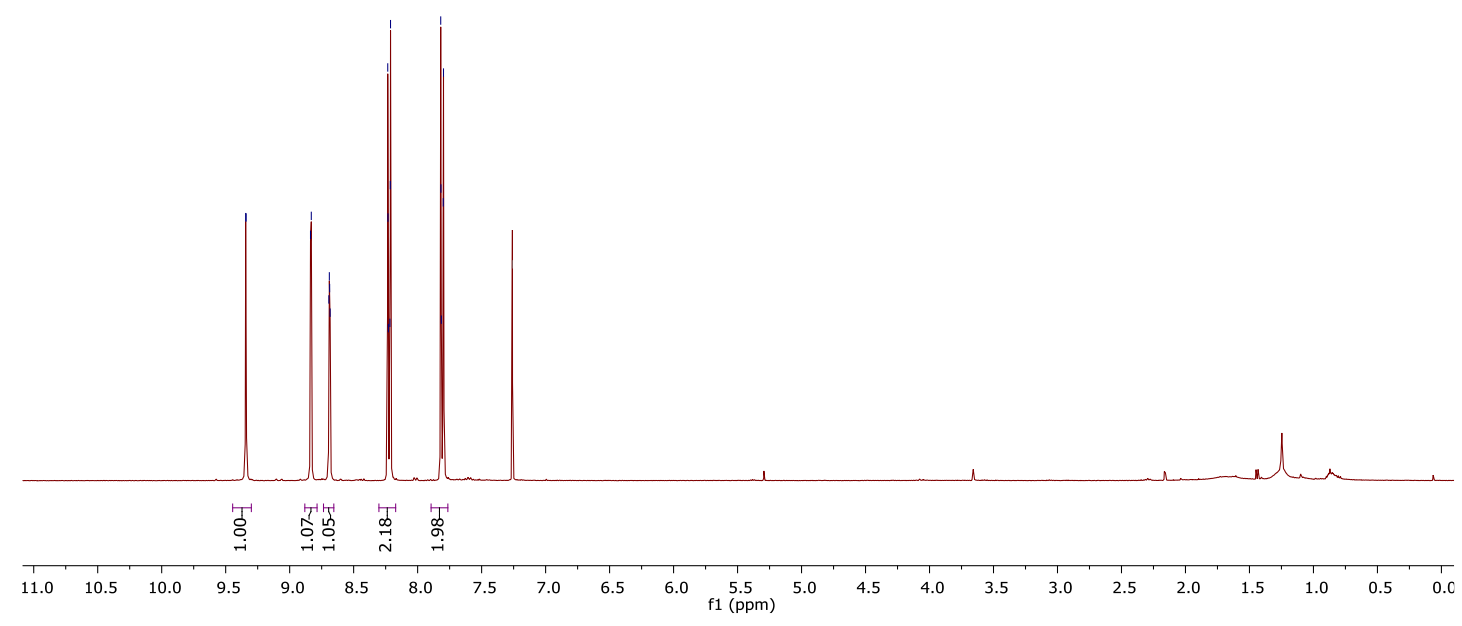

$\hat{0}$
0
0
1

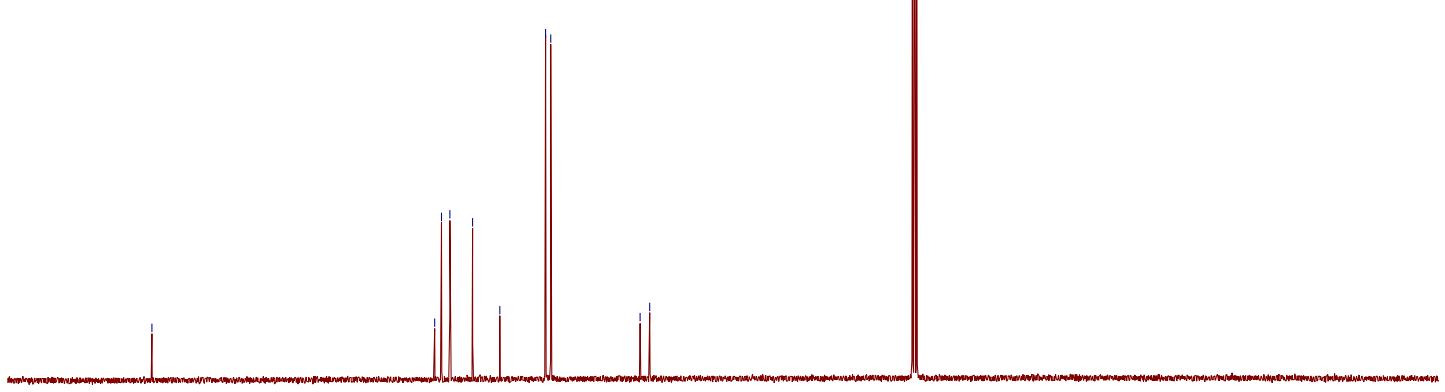

\begin{tabular}{llllllllllllllllllllllllll}
\hline 210 & 200 & 190 & 180 & 170 & 160 & 150 & 140 & 130 & 120 & 110 & 100 & 90 & 80 & 70 & 60 & 50 & 40 & 30 & 20 & 10 & 0
\end{tabular} 
(2,6-dimethoxyphenyl)(pyrimidin-2-yl)methanone (10g)<smiles>COc1cccc(OC)c1C(=O)c1ncccn1</smiles>

Y.

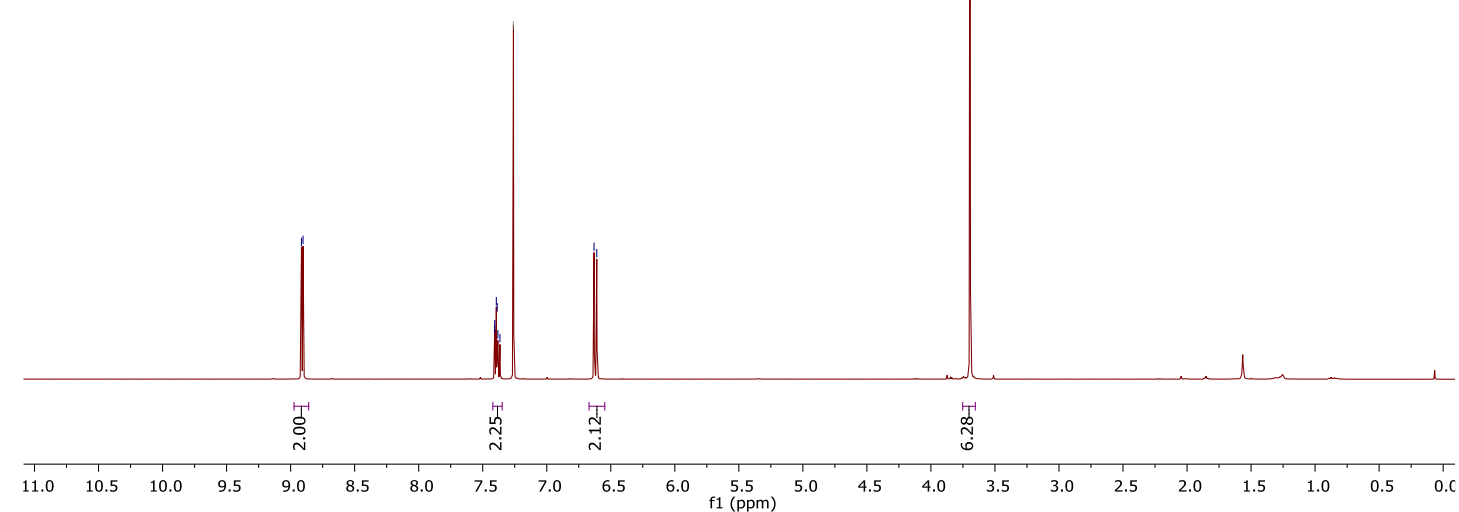

\begin{tabular}{|c|c|c|c|c|}
\hline 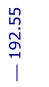 & 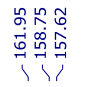 & 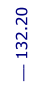 & 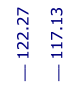 & $\begin{array}{l}0 \\
\stackrel{0}{+} \\
\stackrel{+}{0} \\
1\end{array}$ \\
\hline
\end{tabular}

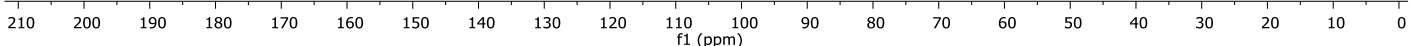


Ethyl 2-(2-methoxyphenyl)-2-oxoacetate (12a)<smiles>CCOC(=O)c1ccccc1OC</smiles>

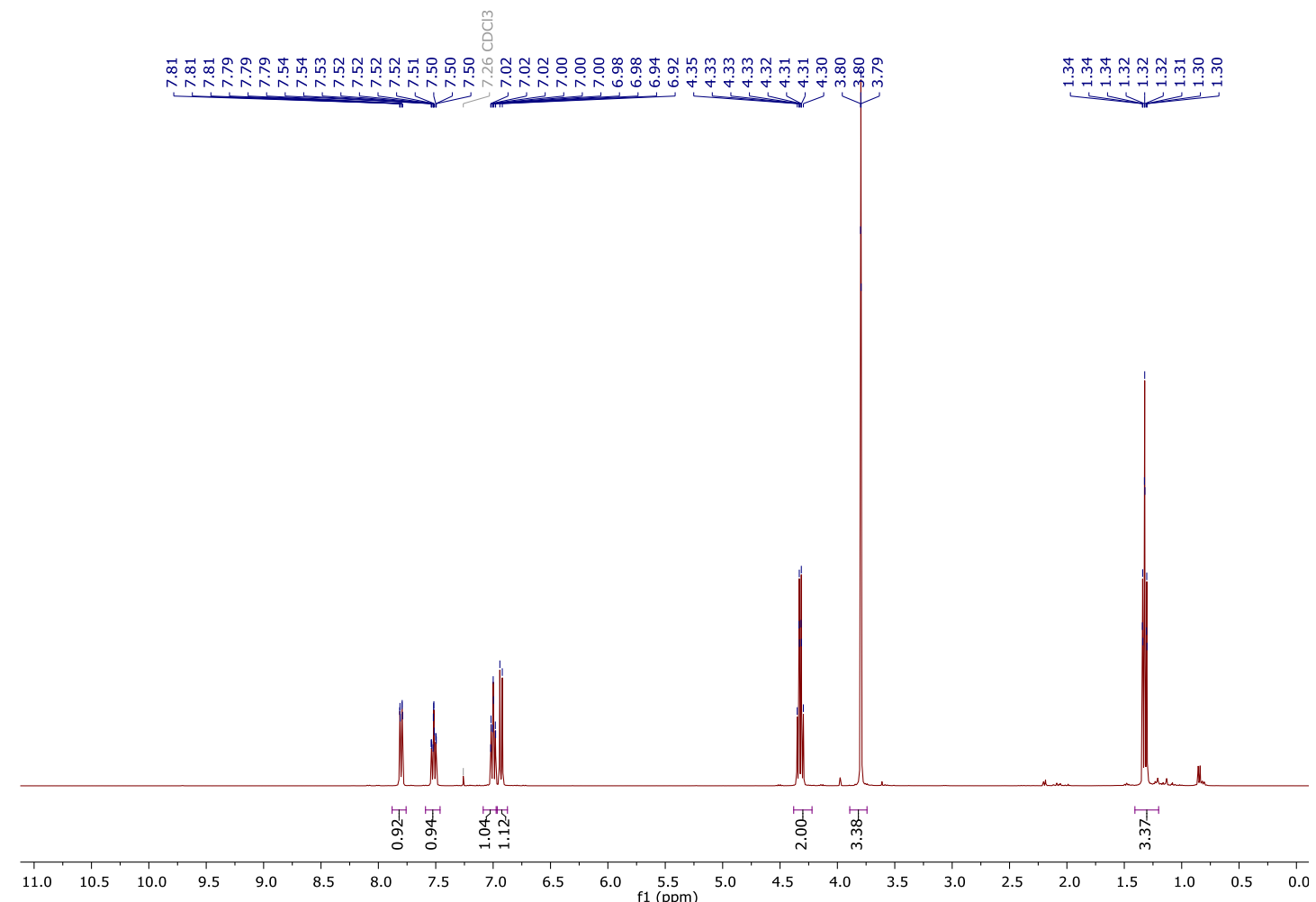

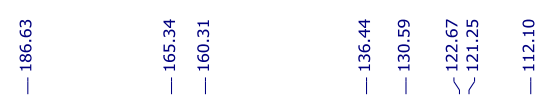

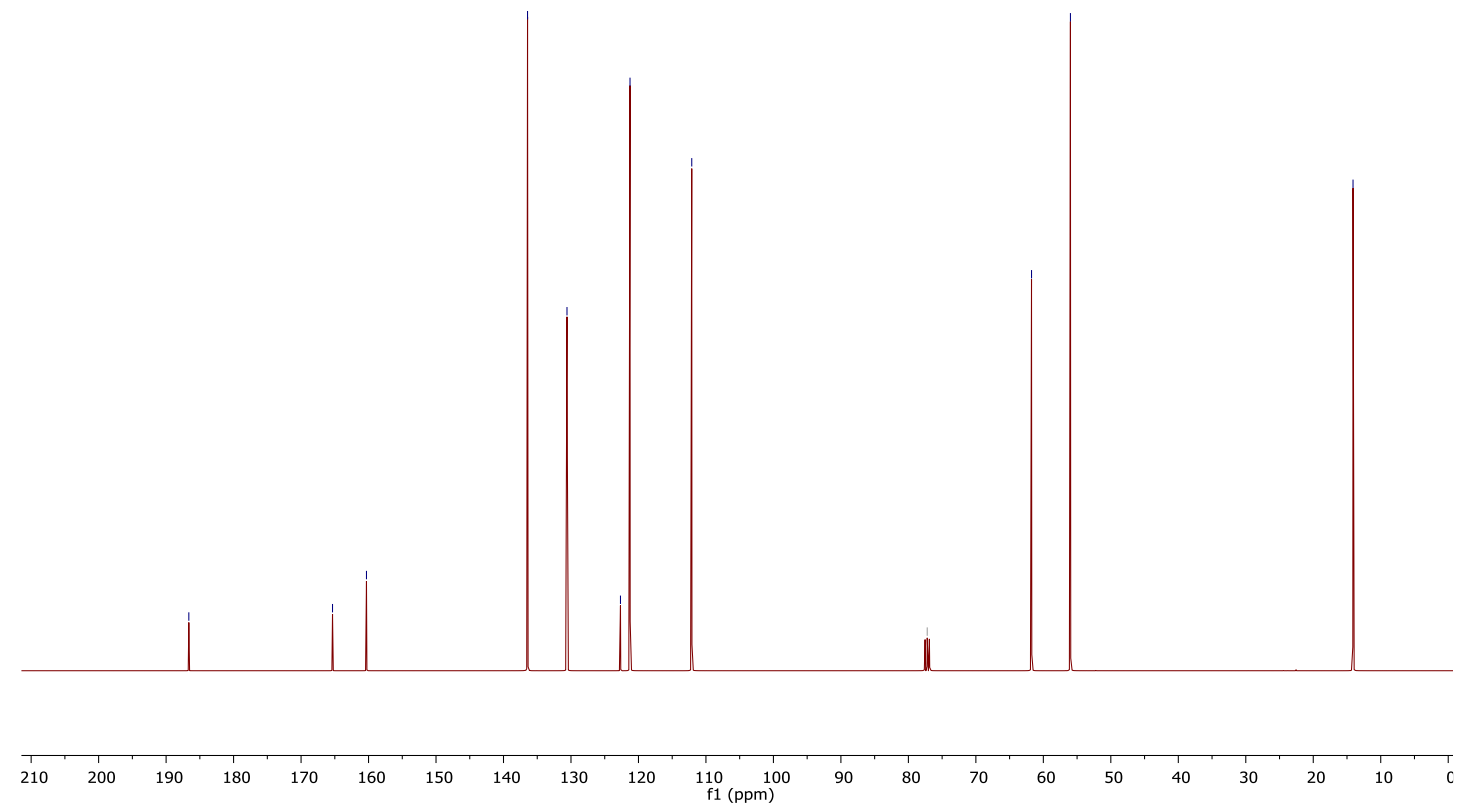


Ethyl 2-(benzo[d][1,3]dioxol-5-yl)-2-oxoacetate (12b)<smiles>CCOC(=O)c1ccc2c(c1)OCO2</smiles>

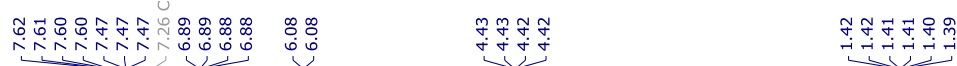

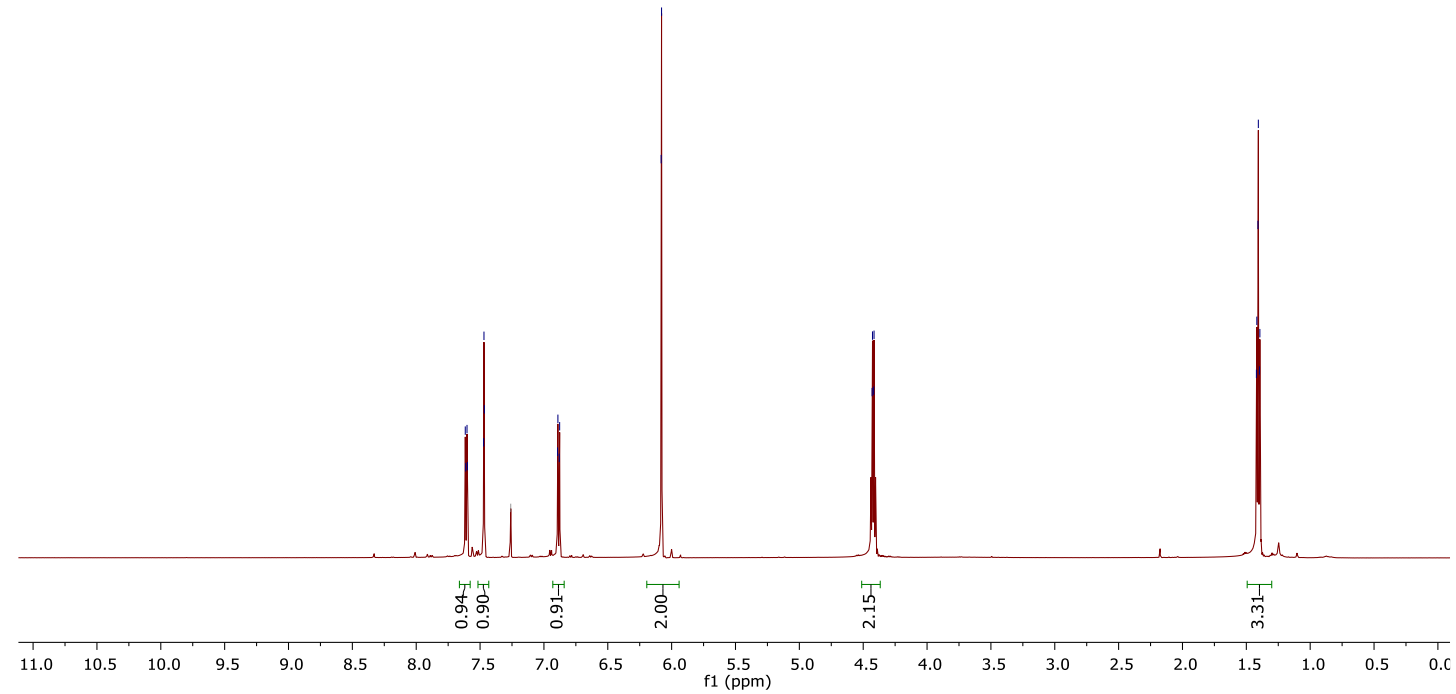

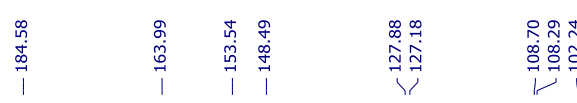
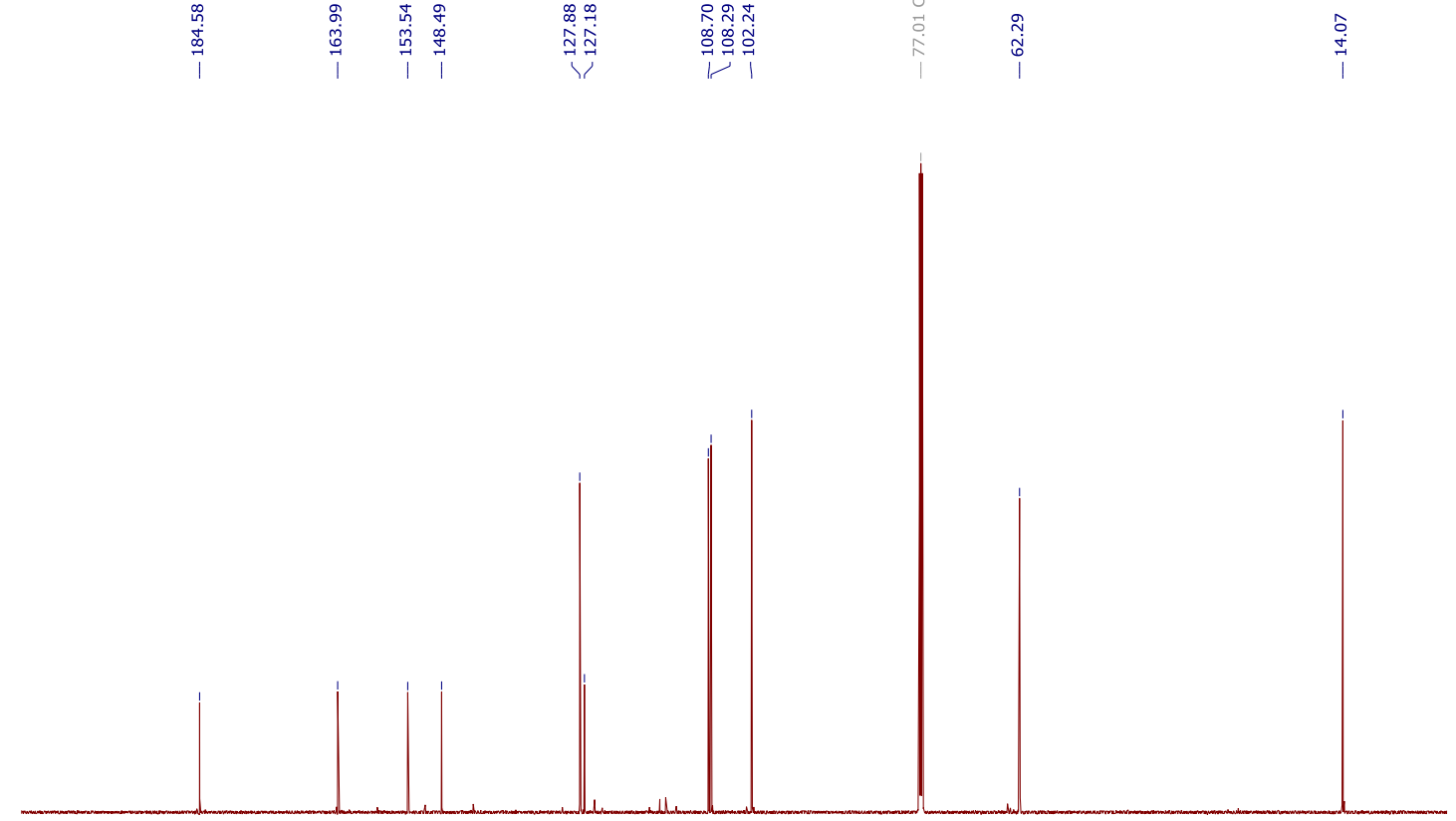

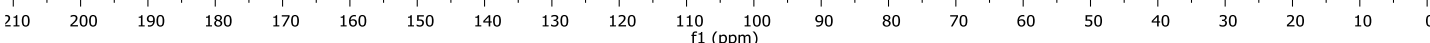


Ethyl 2-(3-fluoro-4-methoxyphenyl)-2-oxoacetate (12c)<smiles>CCOC(=O)c1ccc(OC)c(F)c1</smiles>

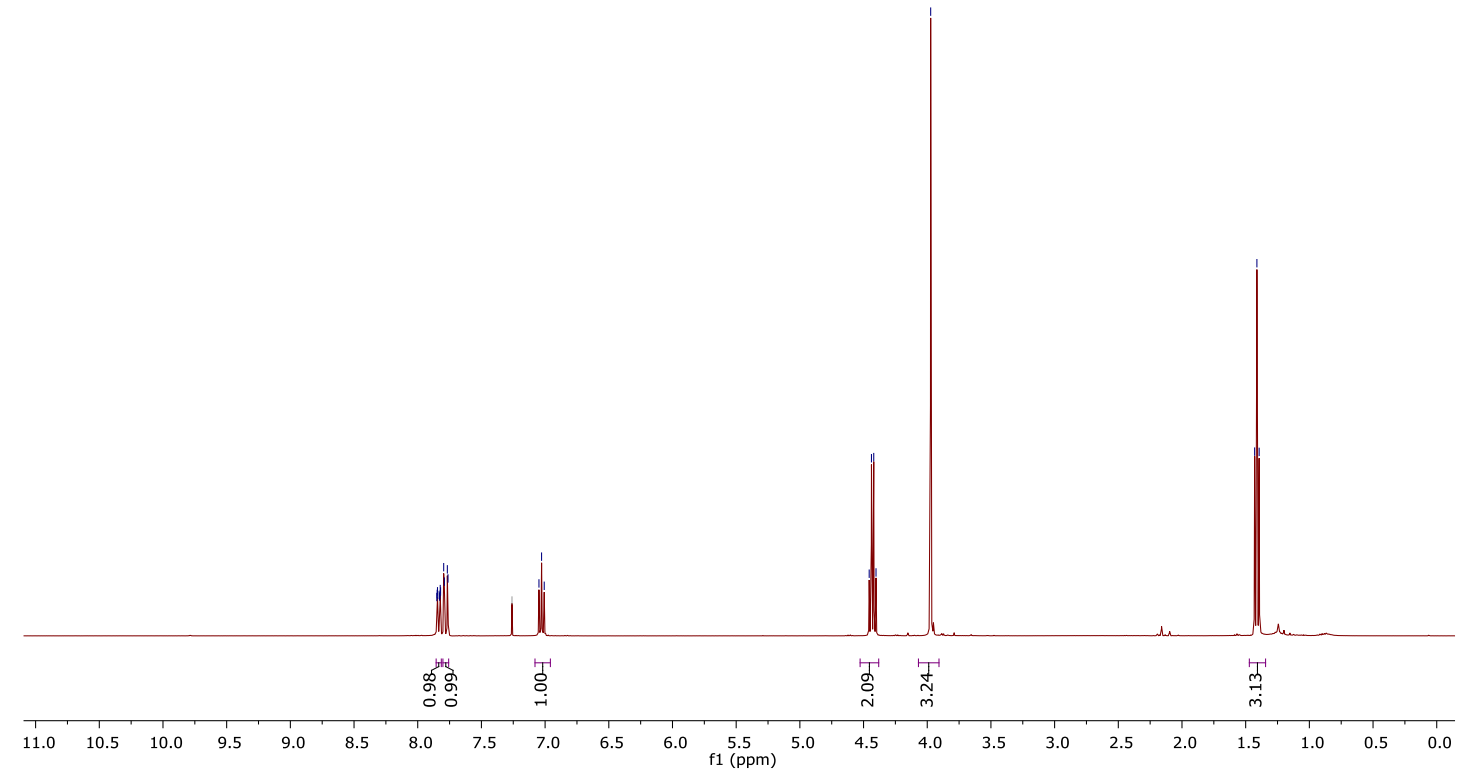

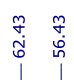

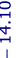

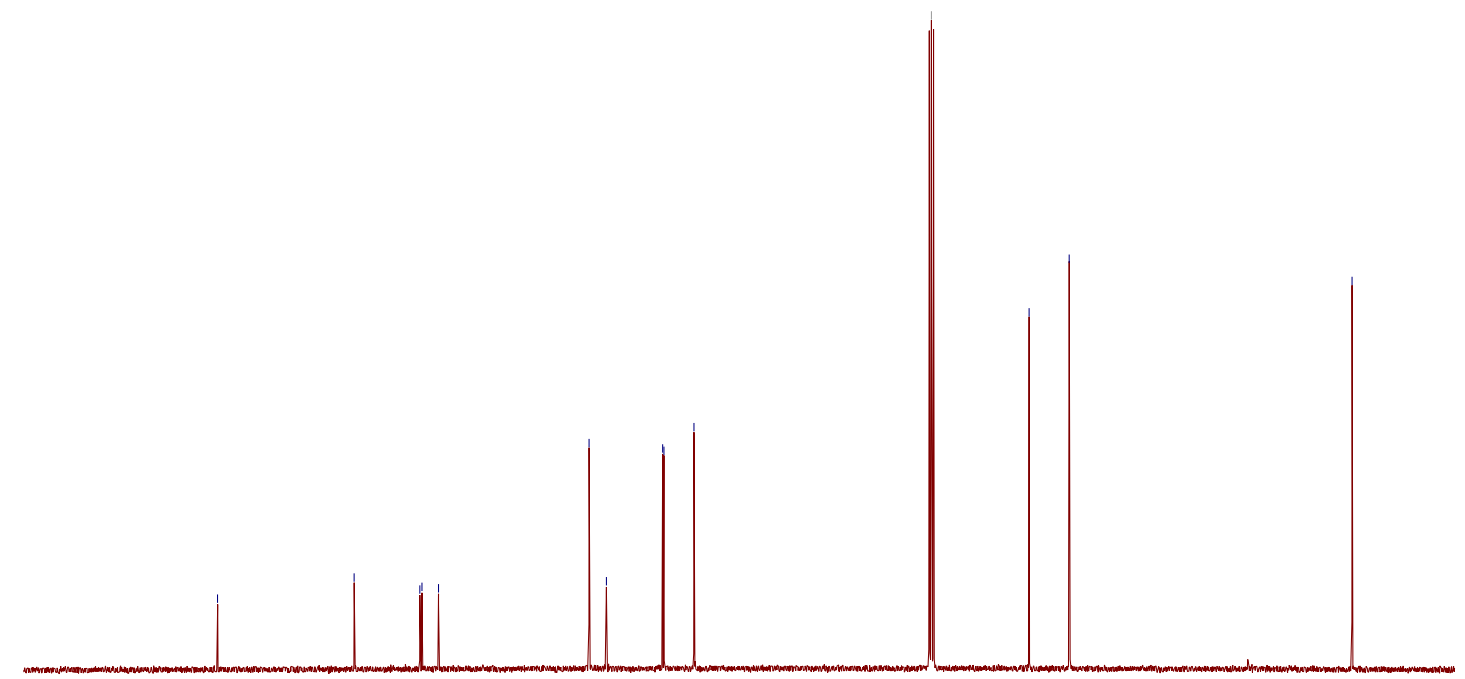

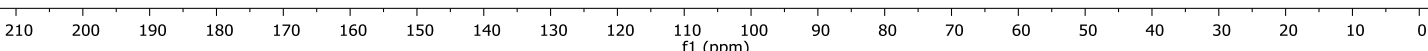


Ethyl 2-(2-fluoro-[1,1'-biphenyl]-4-yl)-2-oxoacetate (12d)<smiles>CCOC(=O)c1ccc(-c2ccccc2)c(F)c1</smiles>

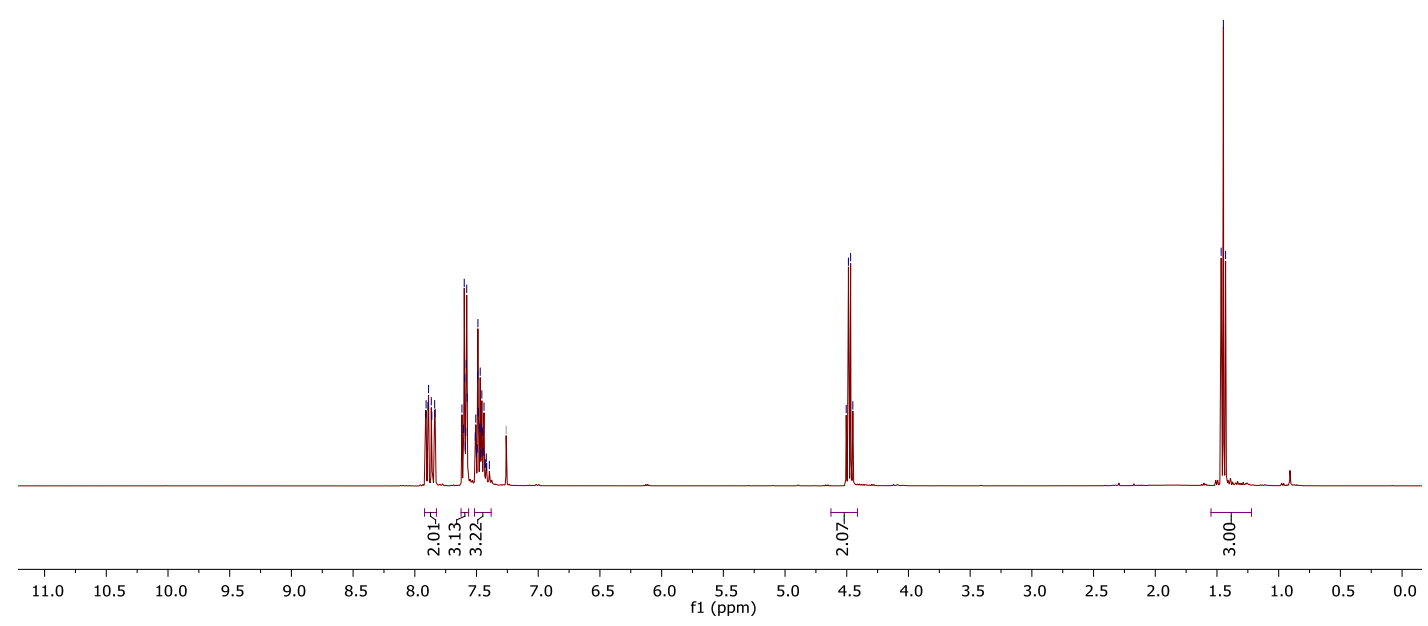

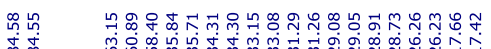

证
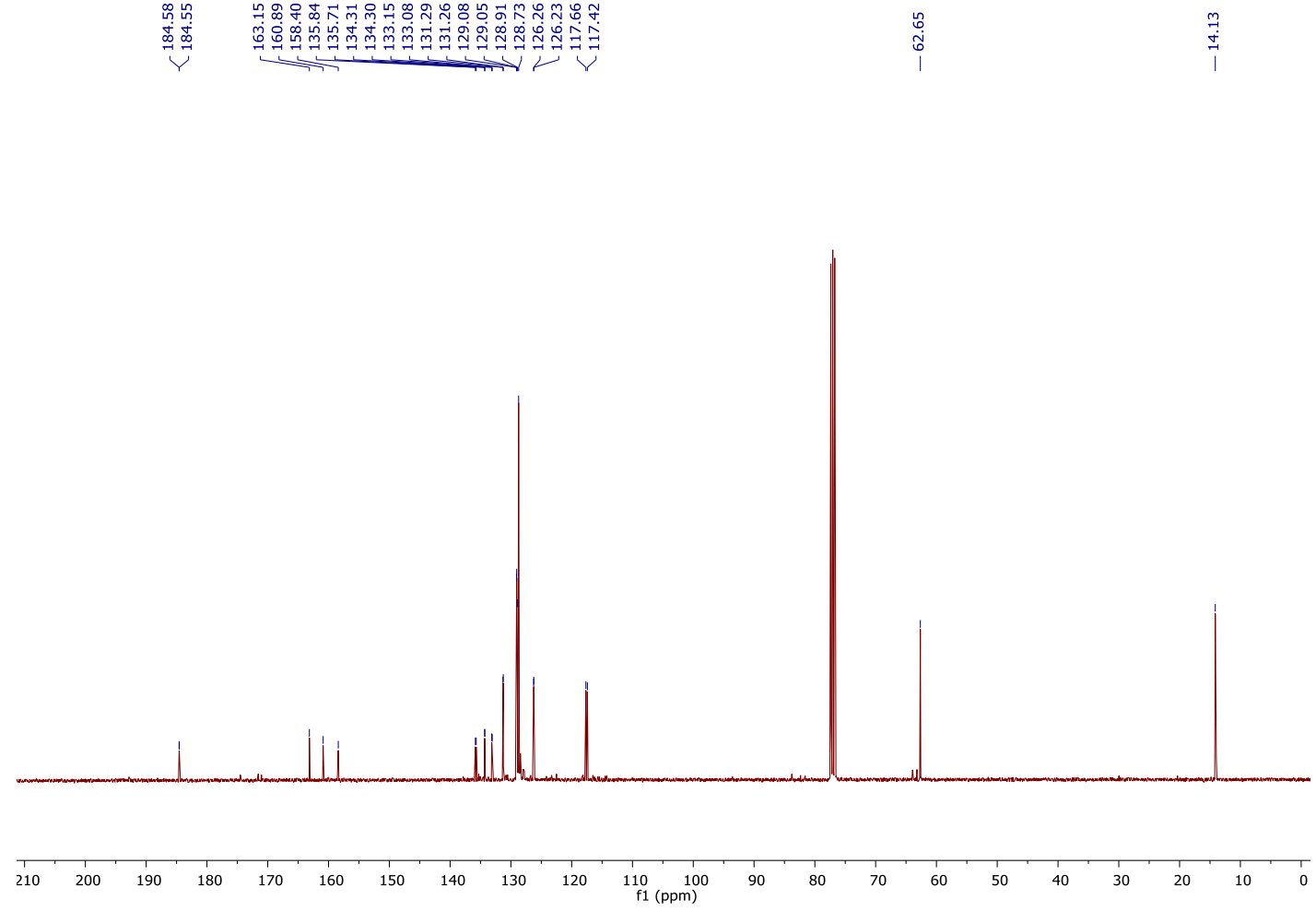
Ethyl 2-oxo-2-(4-(trifluoromethyl)phenyl)acetate (12e)<smiles>CCOC(=O)c1ccc(C(F)(F)F)cc1</smiles>
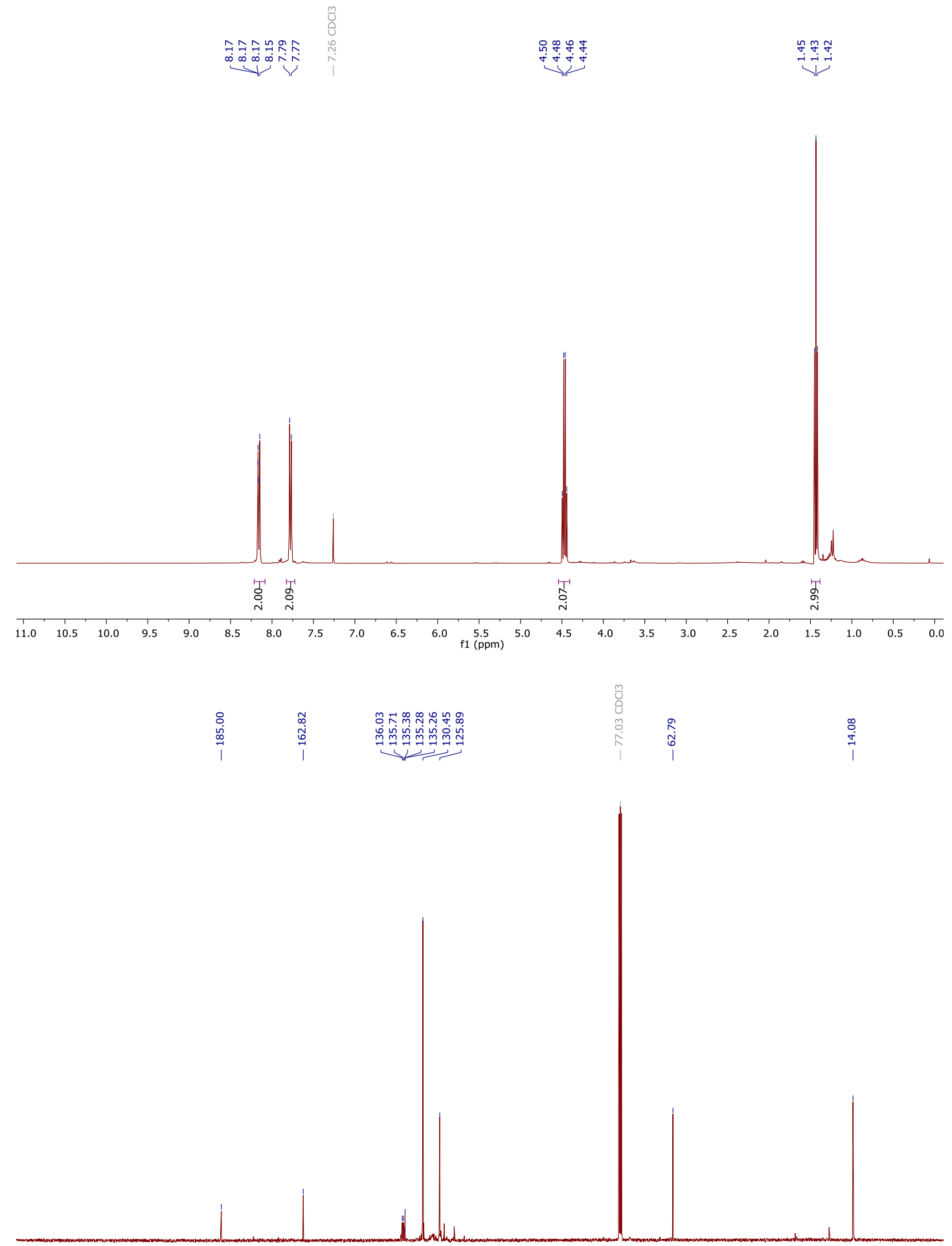

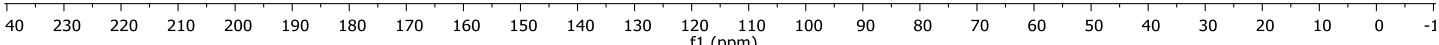




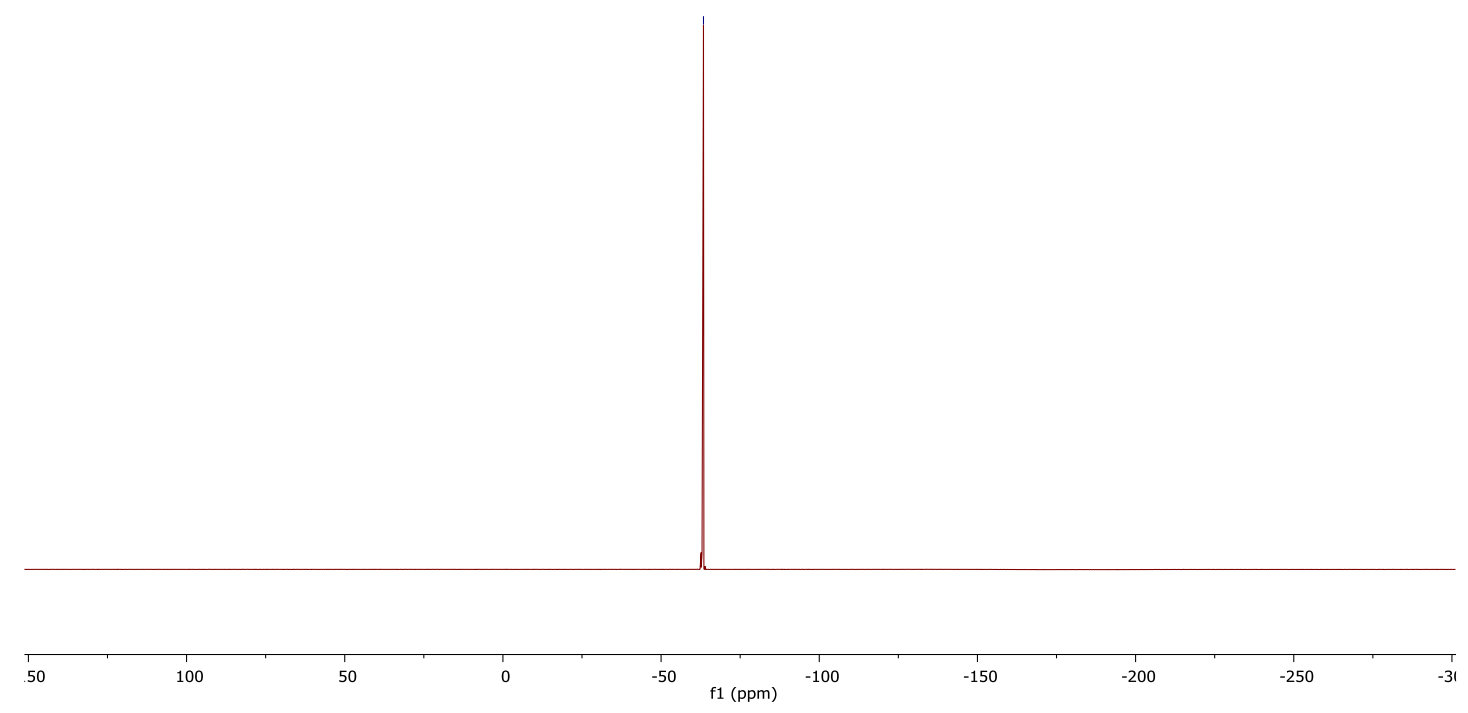


(2-Methoxyphenyl)(phenyl)methanone (16a)<smiles>COc1ccccc1C(=O)c1ccccc1</smiles>

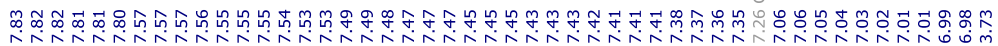
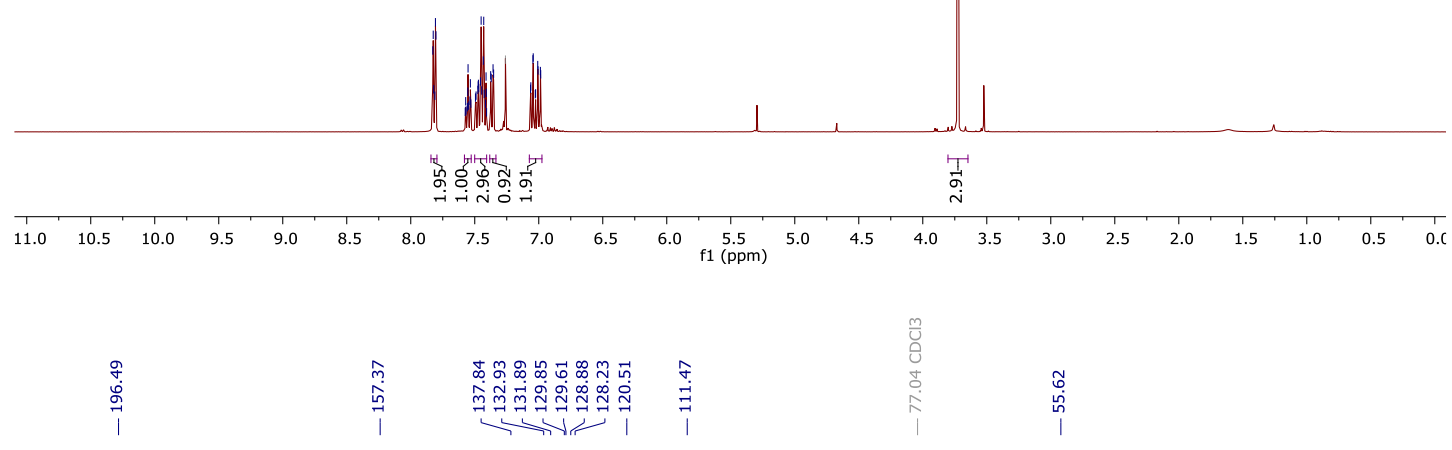

0
0
0
0
0
0

总
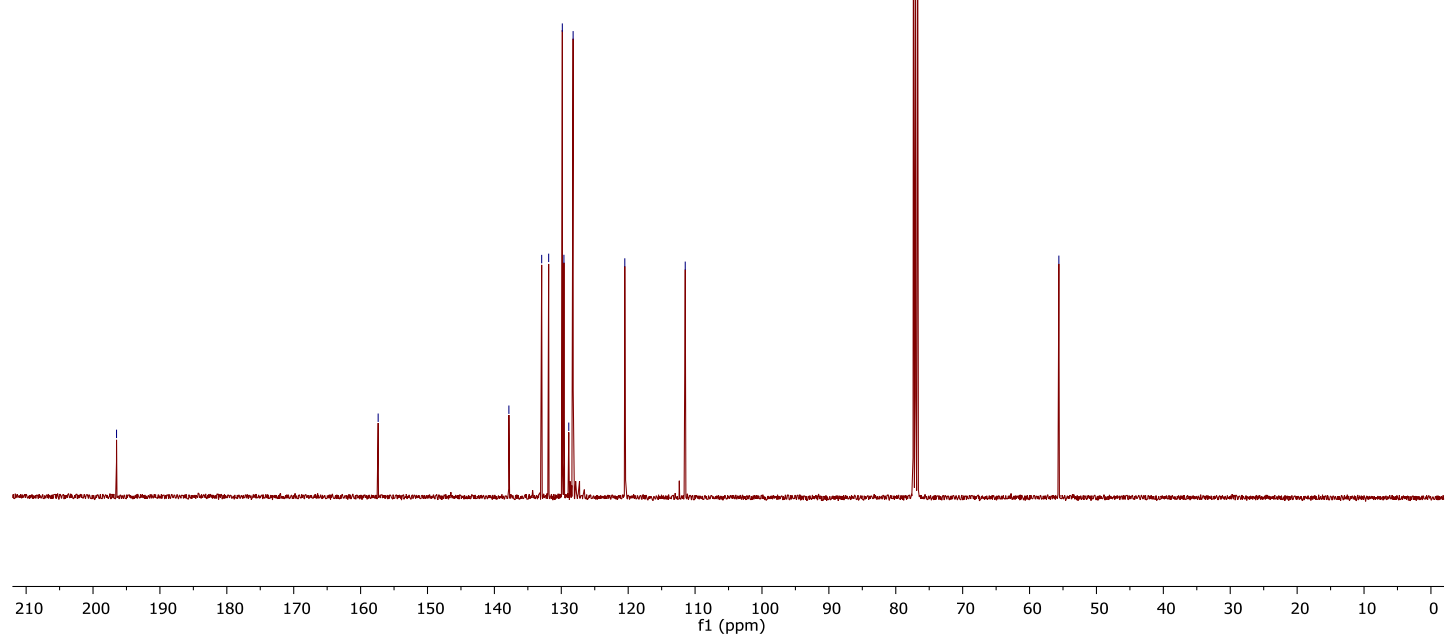

SI-59 
Benzo[d][1,3]dioxol-5-yl(phenyl)methanone (16b)<smiles>O=C(c1ccccc1)c1ccc2c(c1)OCO2</smiles>

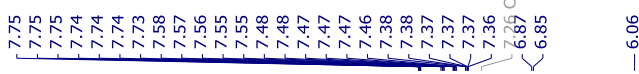

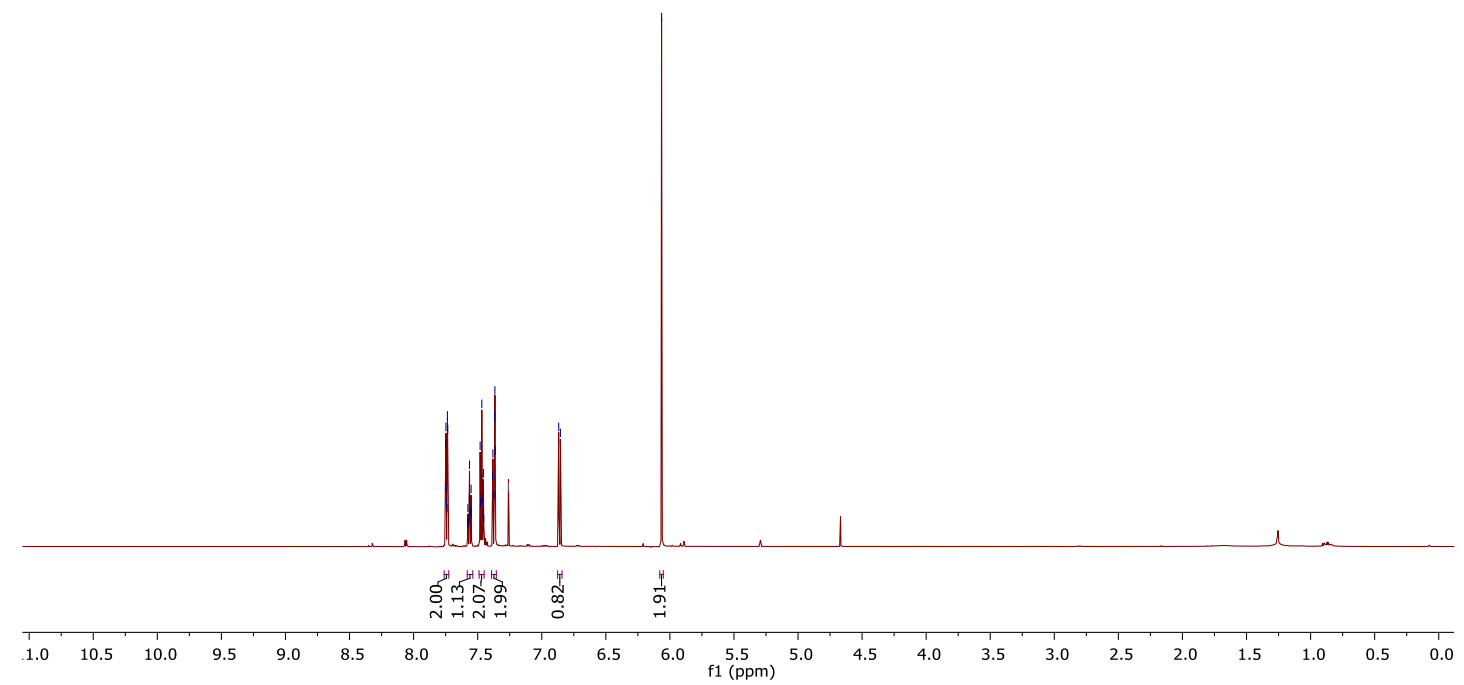

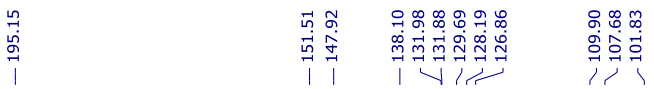

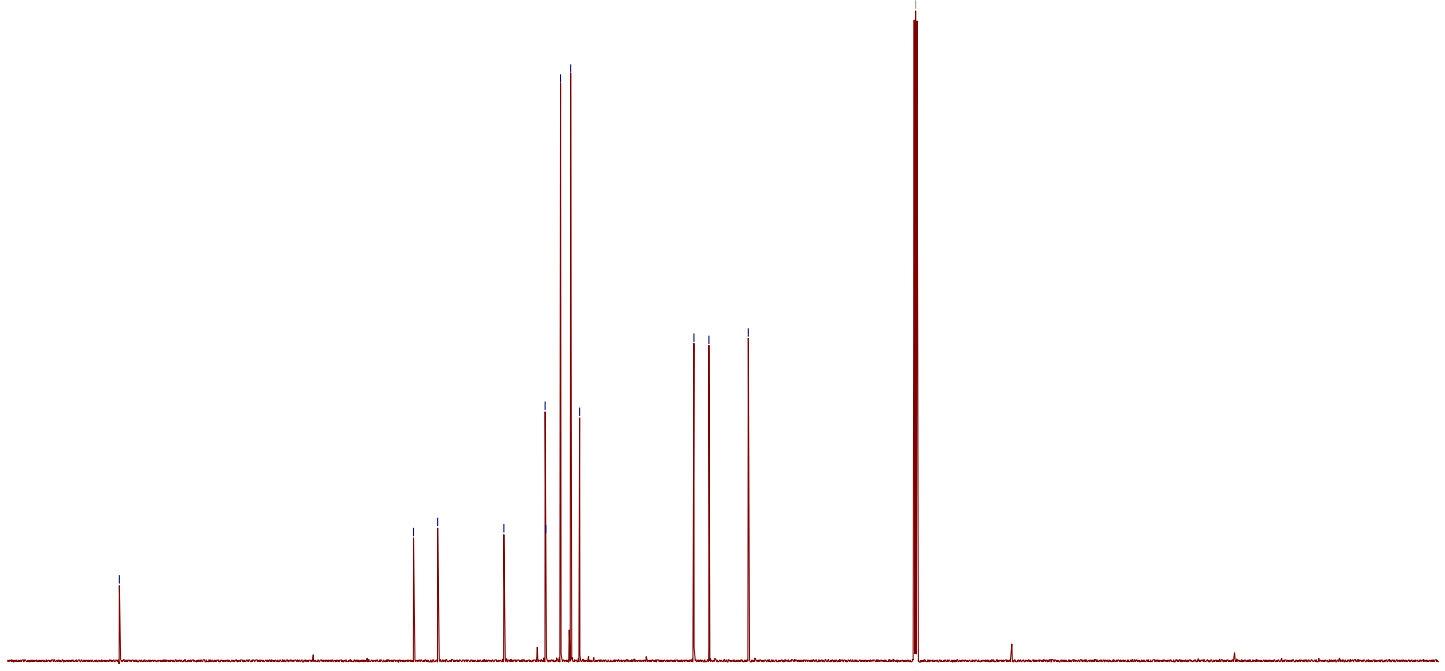

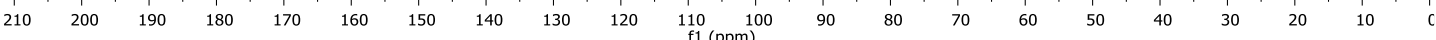


(4-Chlorophenyl)(3,5-dimethoxyphenyl)methanone (16c)<smiles>COc1cc(OC)cc(C(=O)c2ccc(Cl)cc2)c1</smiles>
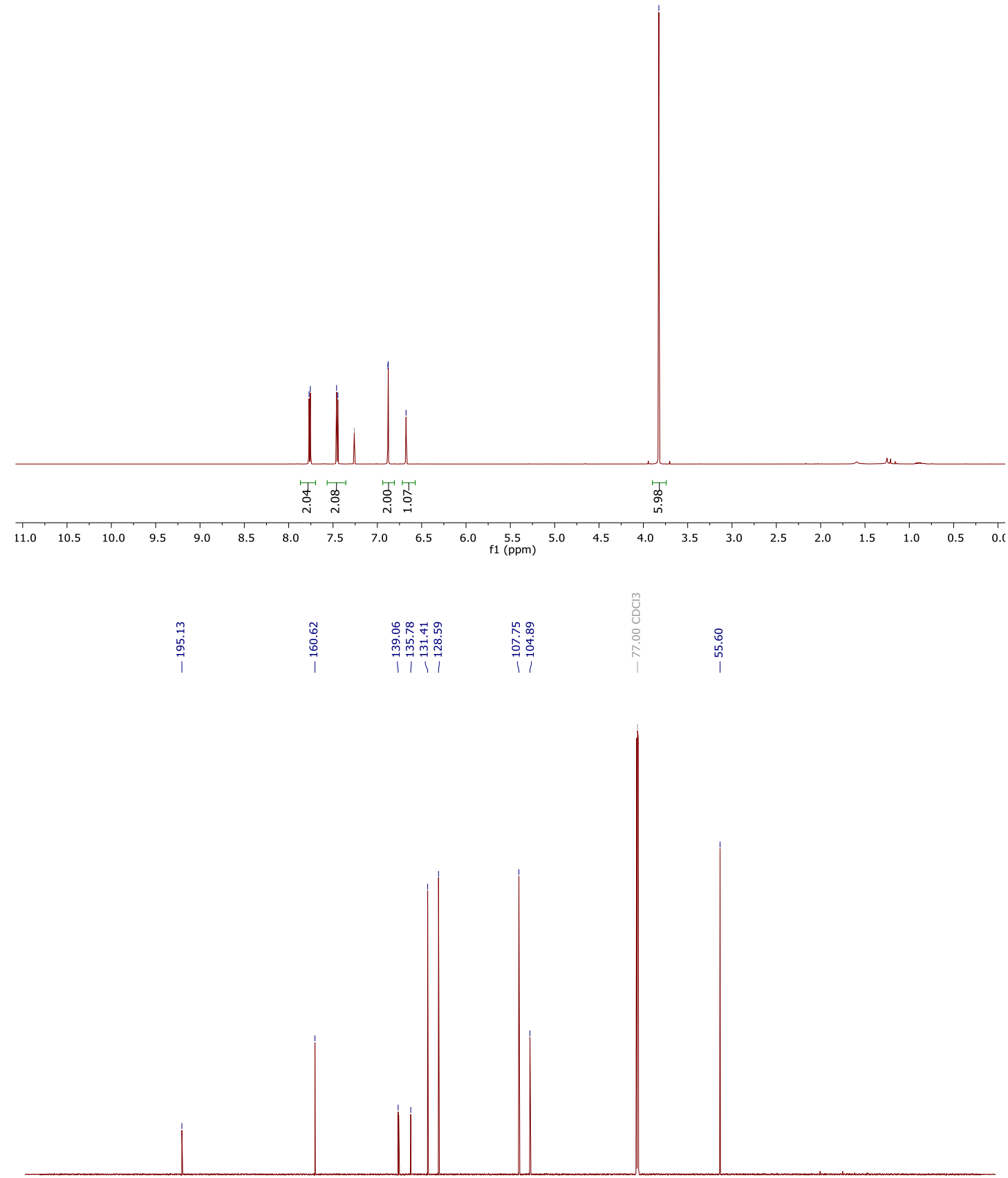

$\begin{array}{llllllllllll}230 & 220 & 210 & 200 & 190 & 180 & 170 & 160 & 150 & 140 & 130 & 120 \\ \mathrm{f} 1(\mathrm{ppm}) & 100\end{array}$ 
(4-(Tert-butyl)phenyl)(4-chlorophenyl)methanone (16d)<smiles>CC(C)(C)c1ccc(C(=O)c2ccc(Cl)cc2)cc1</smiles>

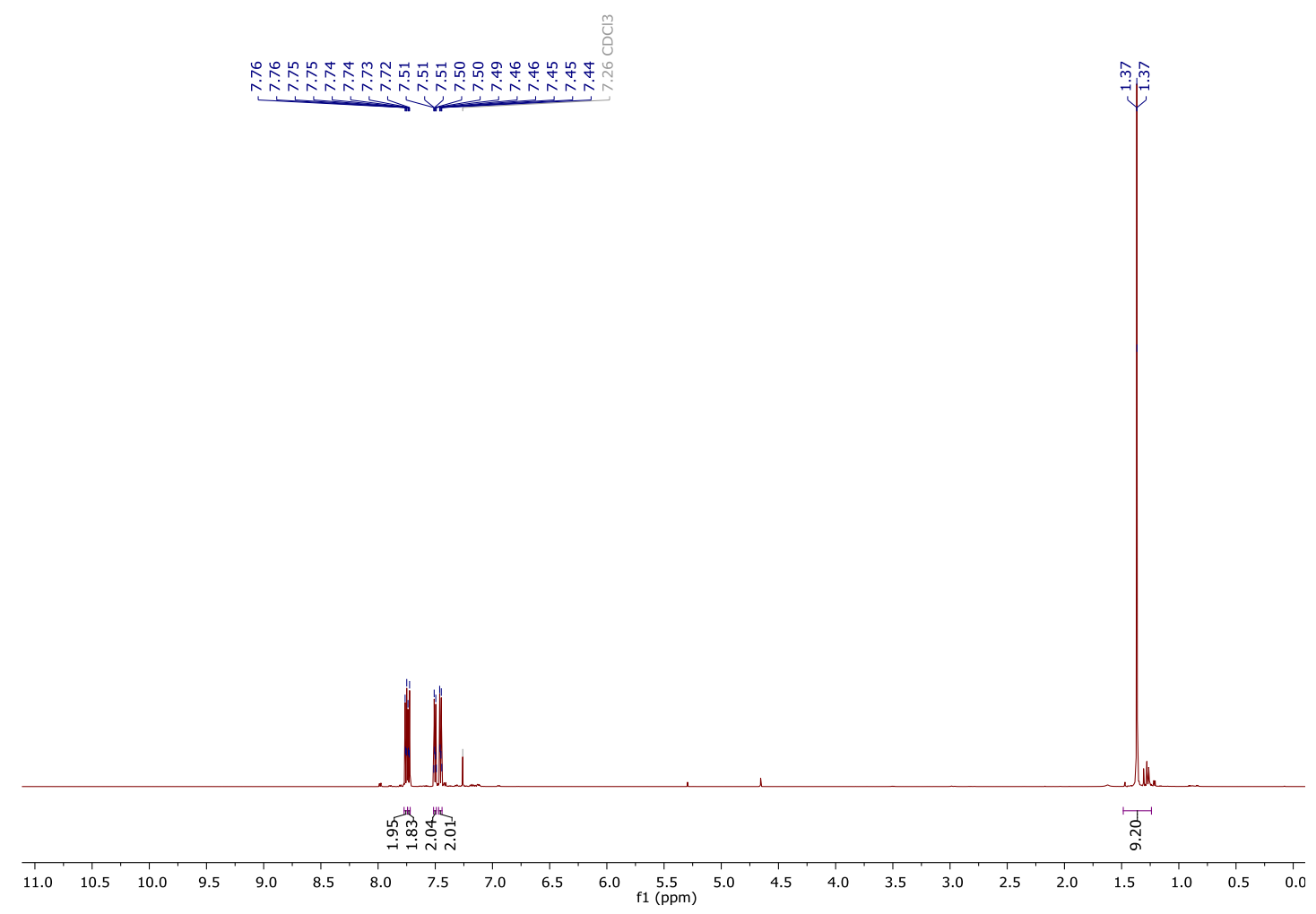

至
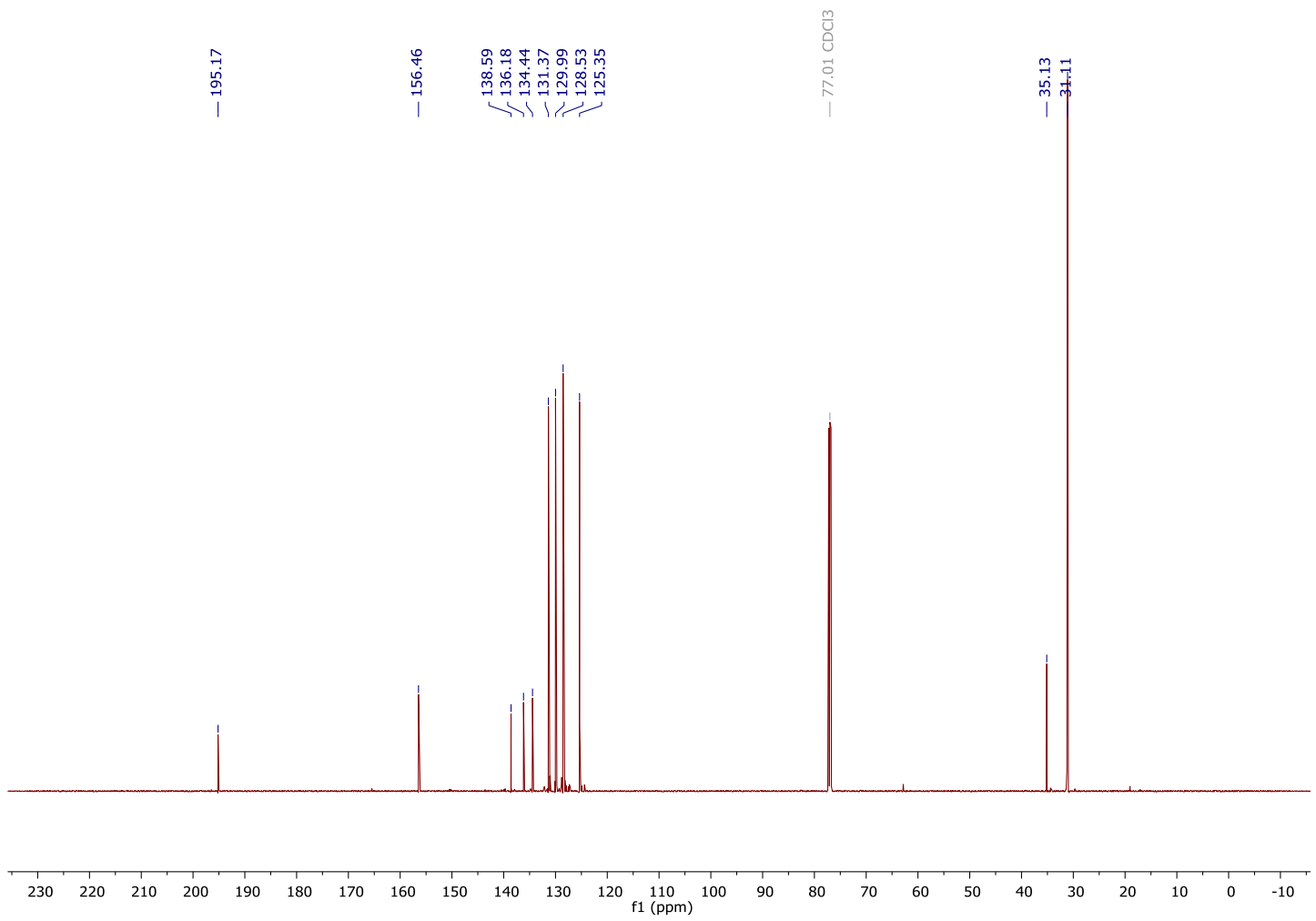
Benzo[d][1,3]dioxol-5-yl(3,4-difluorophenyl)methanone (16e)<smiles>O=C(c1ccc(F)c(F)c1)c1ccc2c(c1)OCO2</smiles>

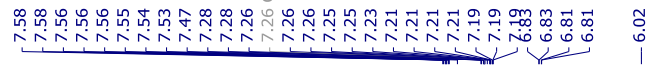

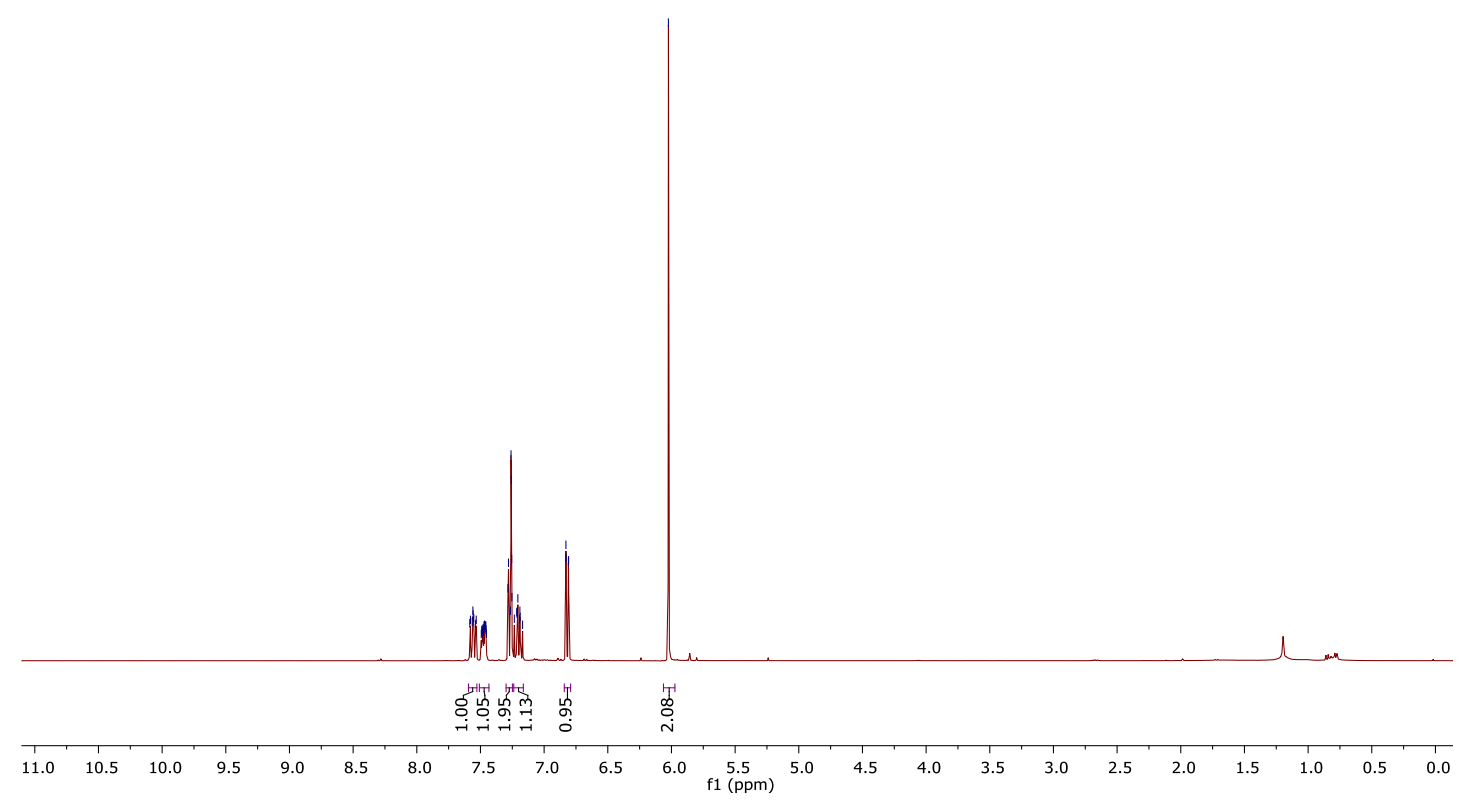

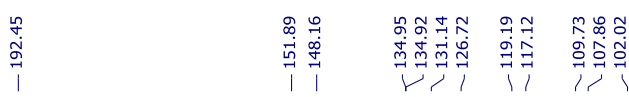

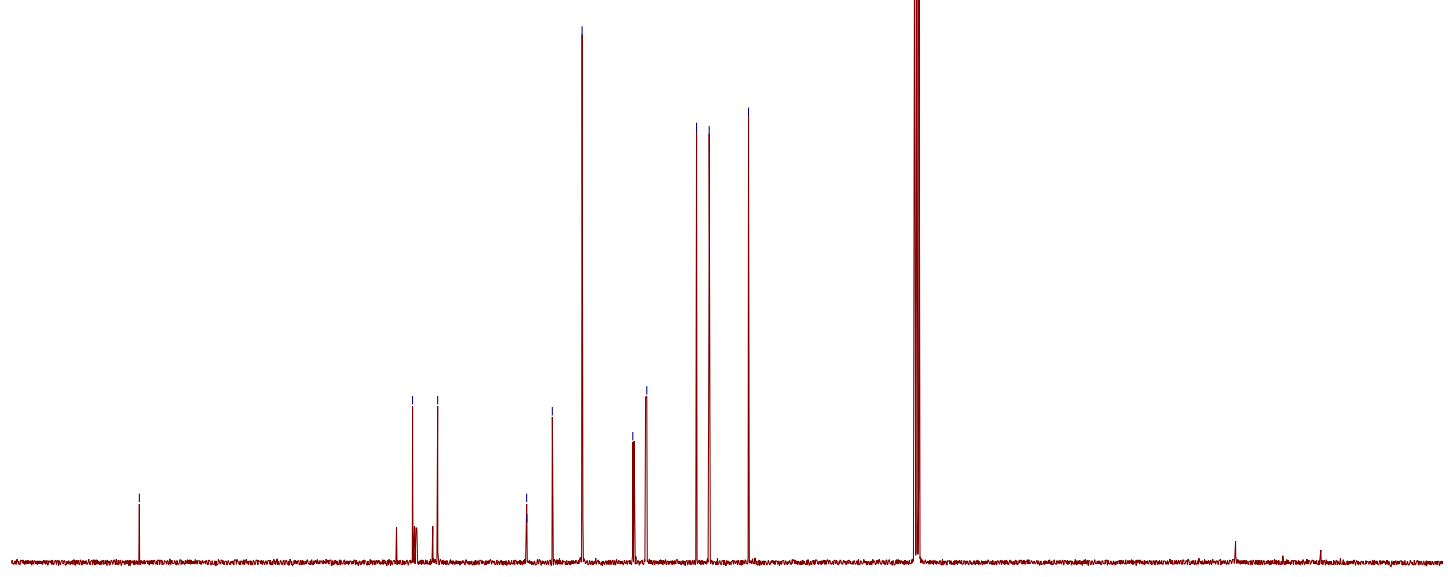

\begin{tabular}{llllllllllllllllllllllllllll}
\hline & 210 & 200 & 190 & 180 & 170 & 160 & 150 & 140 & 130 & 120 & 110 & 100 & 90 & 80 & 70 & 60 & 50 & 40 & 30 & 20 & 10 & 0
\end{tabular} 
(3,4-Difluorophenyl)(1-methyl-1H-indol-5-yl)methanone (16f)<smiles>Cn1ccc2cc(C(=O)c3ccc(F)c(F)c3)ccc21</smiles>

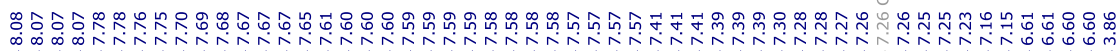

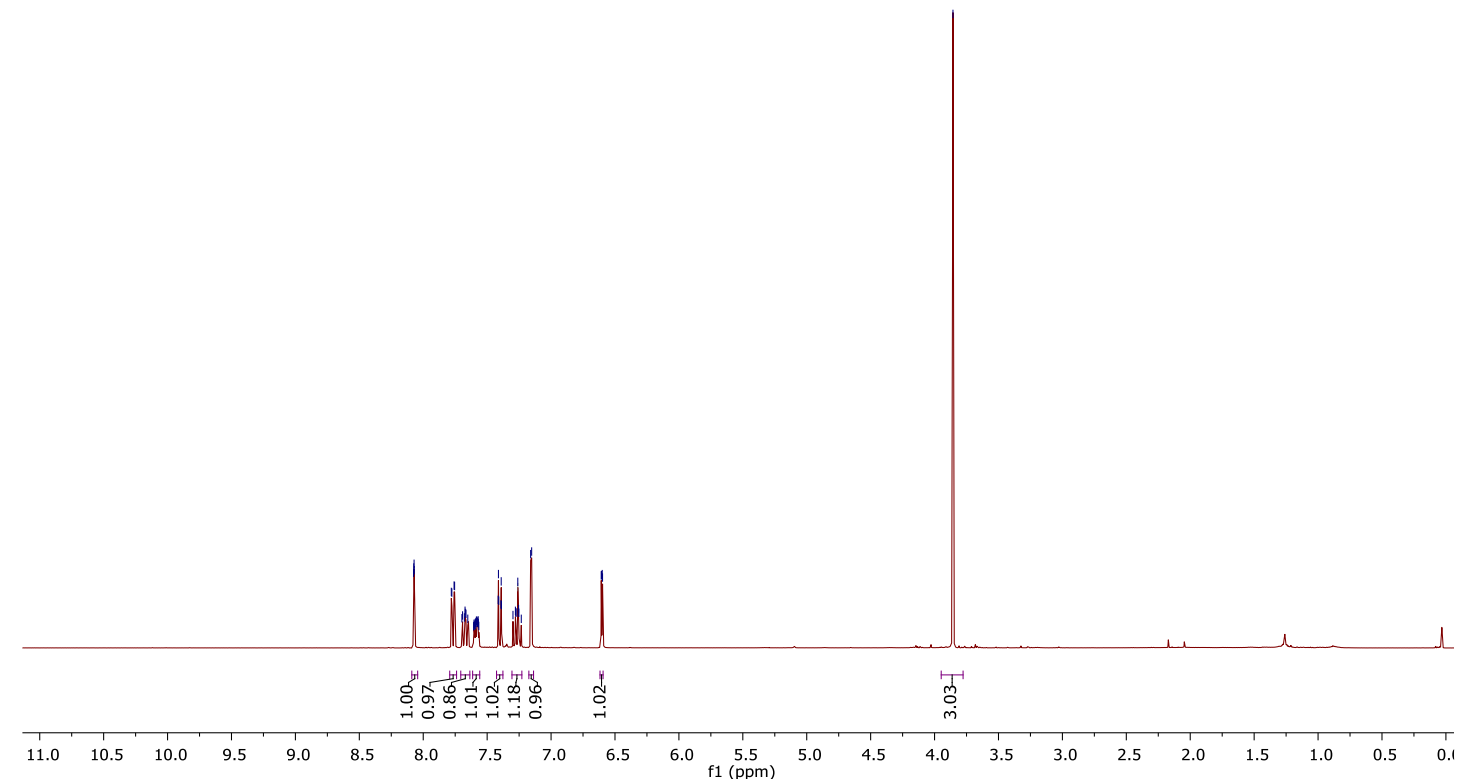

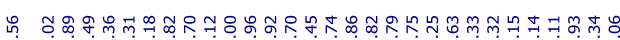

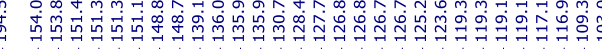

$\stackrel{\substack{m \\ m}}{1}$

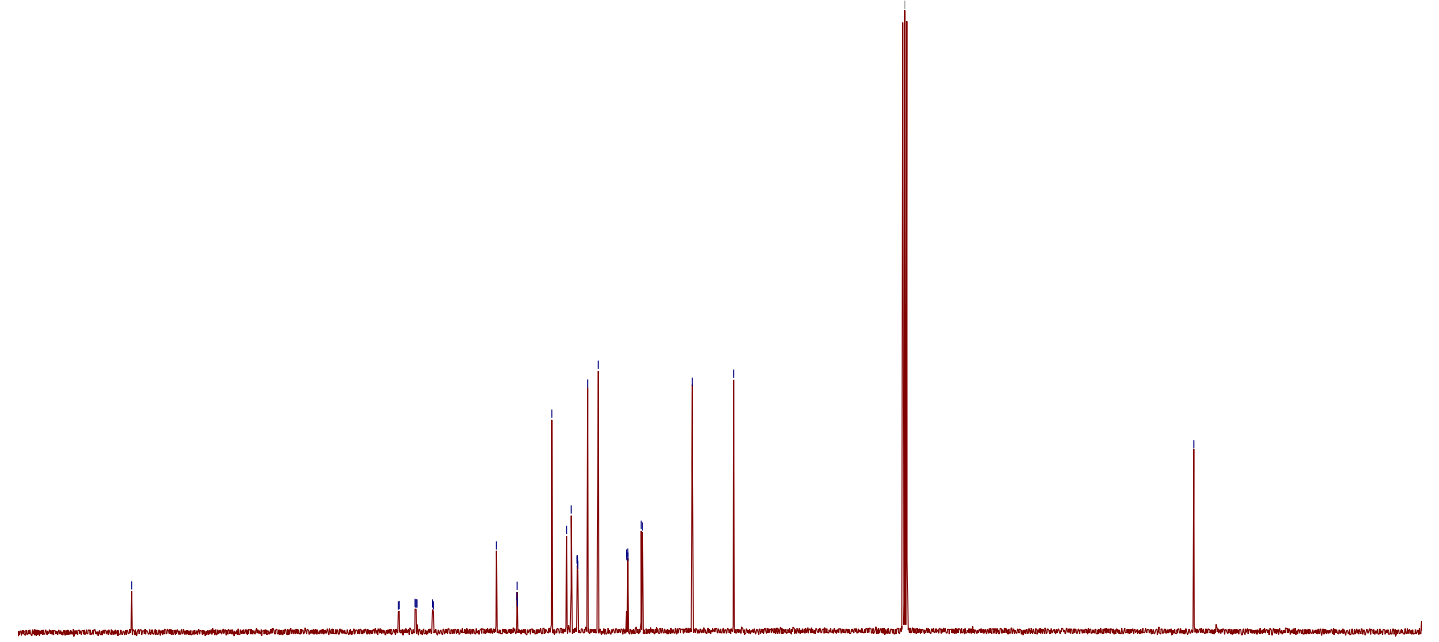

$\begin{array}{lllllllllllllllllllllllllllll} & 210 & 200 & 190 & 180 & 170 & 160 & 150 & 140 & 130 & 120 & 110 & 100 & 90 & 80 & 70 & 60 & 50 & 40 & 30 & 20 & 10 & 0\end{array}$ 
(4-Bromophenyl)(o-tolyl)methanone (16g)<smiles>Cc1ccccc1C(=O)c1ccc(Br)cc1</smiles>
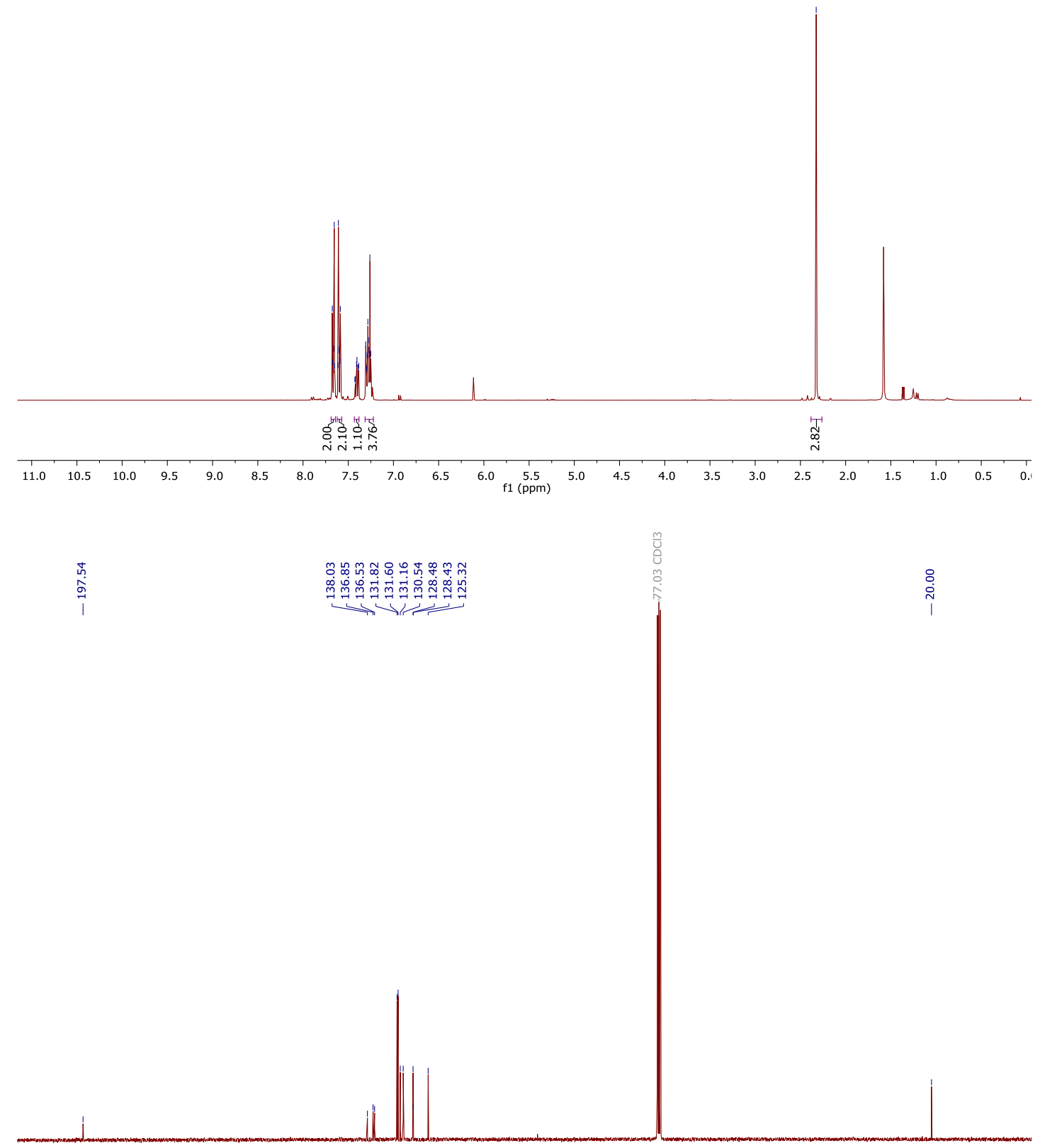

$\begin{array}{llllllllllll}210 & 200 & 190 & 180 & 170 & 160 & 150 & 140 & 130 & 120 & 110 & 100\end{array}$ 
4-(2-Methylbenzoyl)benzonitrile (16h)

CN

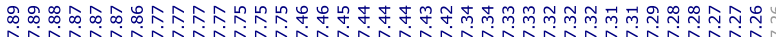
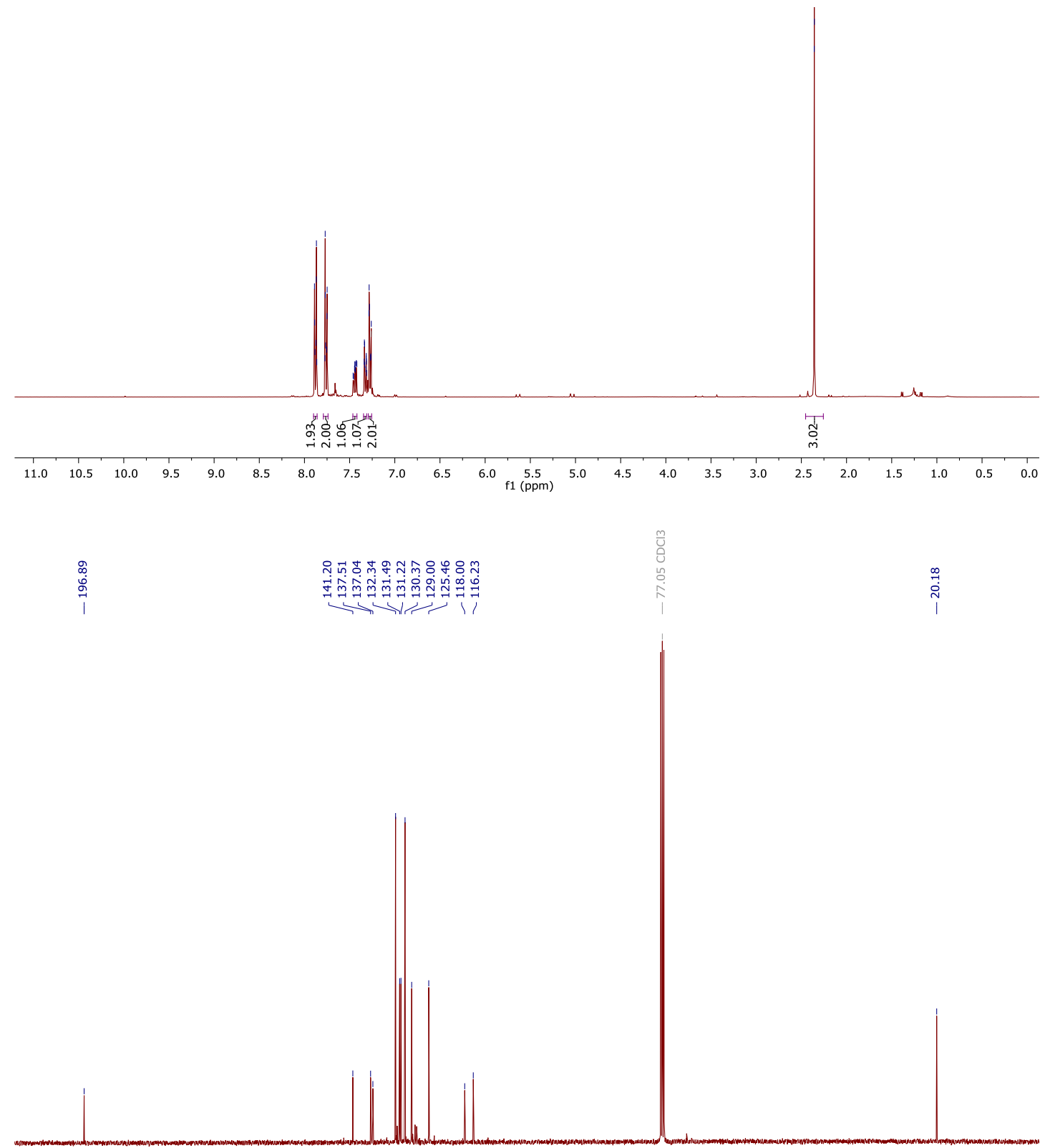

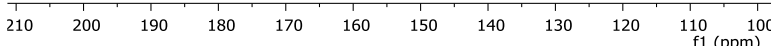


(6-Chloropyridin-3-yl)(3-methoxyphenyl)methanone (16i)<smiles>COc1cccc(C(=O)c2ccc(Cl)nc2)c1</smiles>

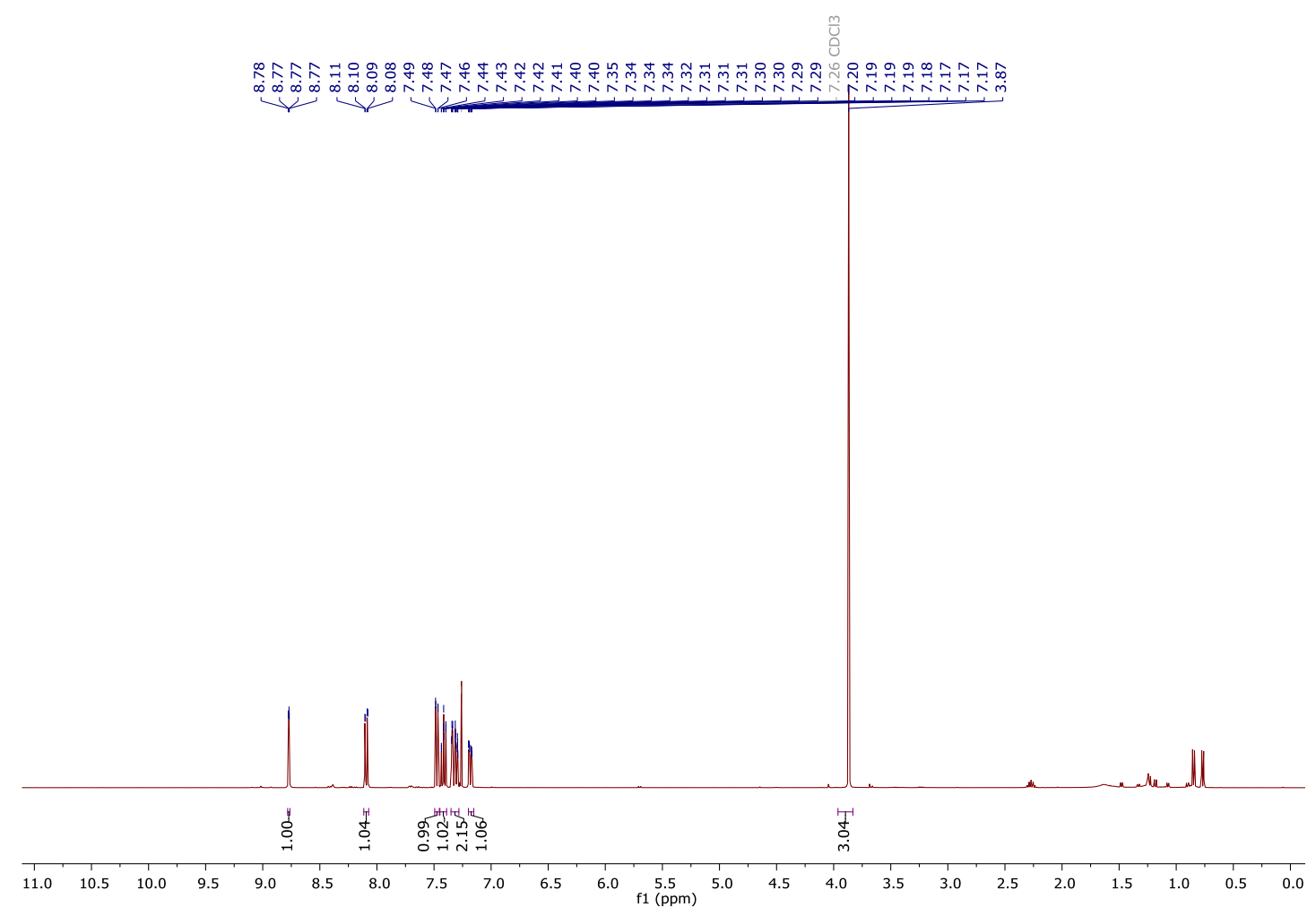

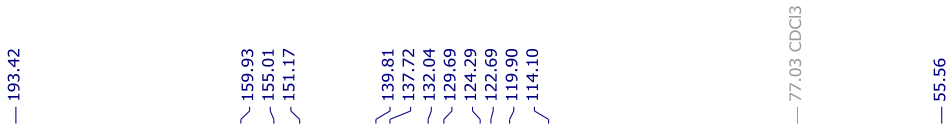

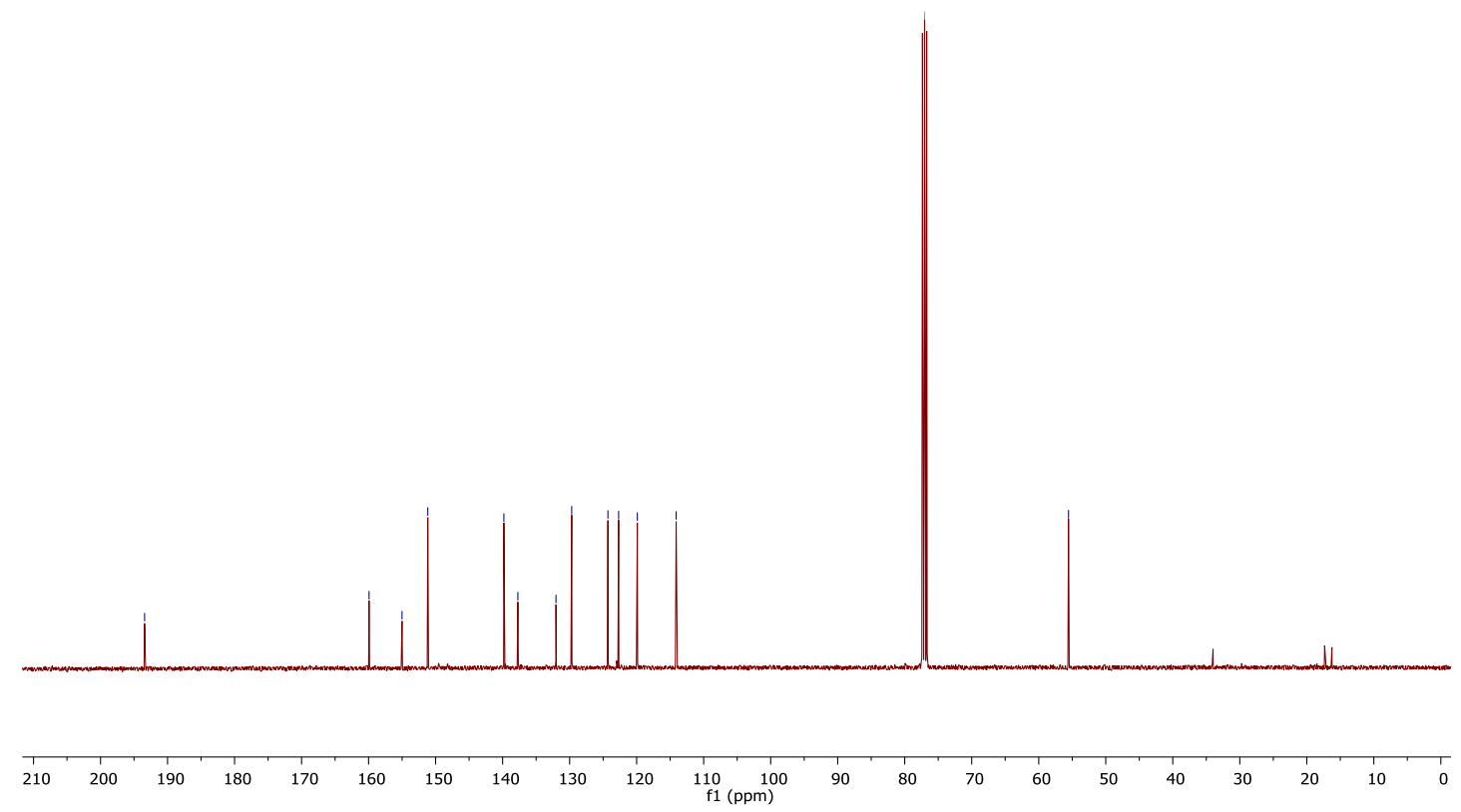

